\title{
O-Allylated Pudovik and Passerini Adducts as Versatile Scaffolds for Product Diversification
}

\author{
Mansour Dolé Kerim, ${ }^{\ddagger}$ Tania Katsina, ${ }^{\dagger}$ Martin Cattoen, ${ }^{\dagger}$ Nicolas Fincias, ${ }^{\ddagger}{ }^{\dagger}$ \\ Stellios Arseniyadis, ${ }^{\dagger}, *$ and Laurent El Kaïm ${ }^{\ddagger} *$ \\ \# Laboratoire de Synthèse Organique, CNRS, Ecole Polytechnique, ENSTA ParisTech \\ UMR 7652, Université Paris-Saclay, 828 Bd des Maréchaux, 91128 Palaiseau (France) \\ E-mail: s.arseniyadis@qmul.ac.uk
}

+ Queen Mary University of London, School of Biological and Chemical Sciences Mile End Road, London, E1 4NS (UK)

E-mail: laurent.elkaim@ensta-paris.fr

\section{Supporting Information}

Material and methods

${ }^{1} \mathrm{H}$ and ${ }^{13} \mathrm{C}$ NMR spectra 


\section{Materials and Methods}

The reactions were run under argon atmosphere in oven-dried glassware unless otherwise specified. All commercially available compounds were purchased from Sigma Aldrich, Fluorochem, or Alfa Aesar, and used as received. Reactions ran at temperatures were conducted in sealed microwave tubes in an acetone bath which temperature was controlled by a cryostat. Analytical thin layer chromatography (TLC) was performed on silica gel plates (Merck 60F254) visualized either with a UV lamp (254 $\mathrm{nm}$ ) or by using solutions or $\mathrm{KMnO}_{4} / \mathrm{K}_{2} \mathrm{CO}_{3} / \mathrm{AcOH}$ in water followed by heating. Flash chromatographies were performed on silica gel (60-230 mesh). Organic extracts were dried over anhydrous $\mathrm{MgSO}_{4} \cdot{ }^{1} \mathrm{H} \mathrm{NMR}$ spectra were recorded on a Bruker AVANCE 400 at $400 \mathrm{MHz}$ in $\mathrm{CDCl}_{3}$ and the observed signals are reported as follows: chemical shift in parts per million from tetramethylsilane with the solvent as an internal indicator $\left(\mathrm{CDCl}_{3} \delta 7.26 \mathrm{ppm}\right)$, multiplicity ( $\mathrm{s}=$ singlet, $\mathrm{d}=$ doublet, $\mathrm{t}=$ triplet, $\mathrm{q}=$ quartet, $\mathrm{p}=$ pentet, $\mathrm{m}=$ multiplet or overlap of nonequivalent resonances, $\mathrm{br}=$ broad), integration. ${ }^{13} \mathrm{C} \mathrm{NMR}$ spectra were recorded at $101 \mathrm{MHz}$ in $\mathrm{CDCl}_{3}$ and the observed signals were reported as follows: chemical shift in parts per million from tetramethylsilane with the solvent as an internal indicator $\left(\mathrm{CDCl}_{3} \delta 77.0 \mathrm{ppm}\right)$. Coupling constants, J, are reported in Hertz $(\mathrm{Hz})$. All NMR spectra were obtained at room temperature. Mass spectra (MS) were recorded using a HDMS Synapt G2Si Mass spectrometer (Waters). High Performance Liquid Chromatography (HPLC) was carried out on an Agilent system using chiral columns (Daicel). IR spectra were recorded on a Cary 630 FTIR (Agilent Technologies). Specific rotations were recorded on a AA-100 Polarimeter (Optical Activity Ltd). All commercially available compounds were used without further purification. Compounds 2a-I, 2o-r, 2t, 5a-i, 5o, 5q, 5t-u, 5w, 12a-g, 13a-g and 14a-g have already been reported and therefore won't be described here; the spectral data matched those reported in the literature. Compounds $\mathbf{2} \mathbf{r}$ and $\mathbf{2} \mathbf{u}$ were prepared and engaged in the allylation step without further purification. 
PO(OEt) ${ }^{1} \mathrm{H}-\mathrm{NMR}$ of compound $2 \mathrm{~m}\left(400 \mathrm{MHz}, \mathrm{CDCl}_{3}\right)$

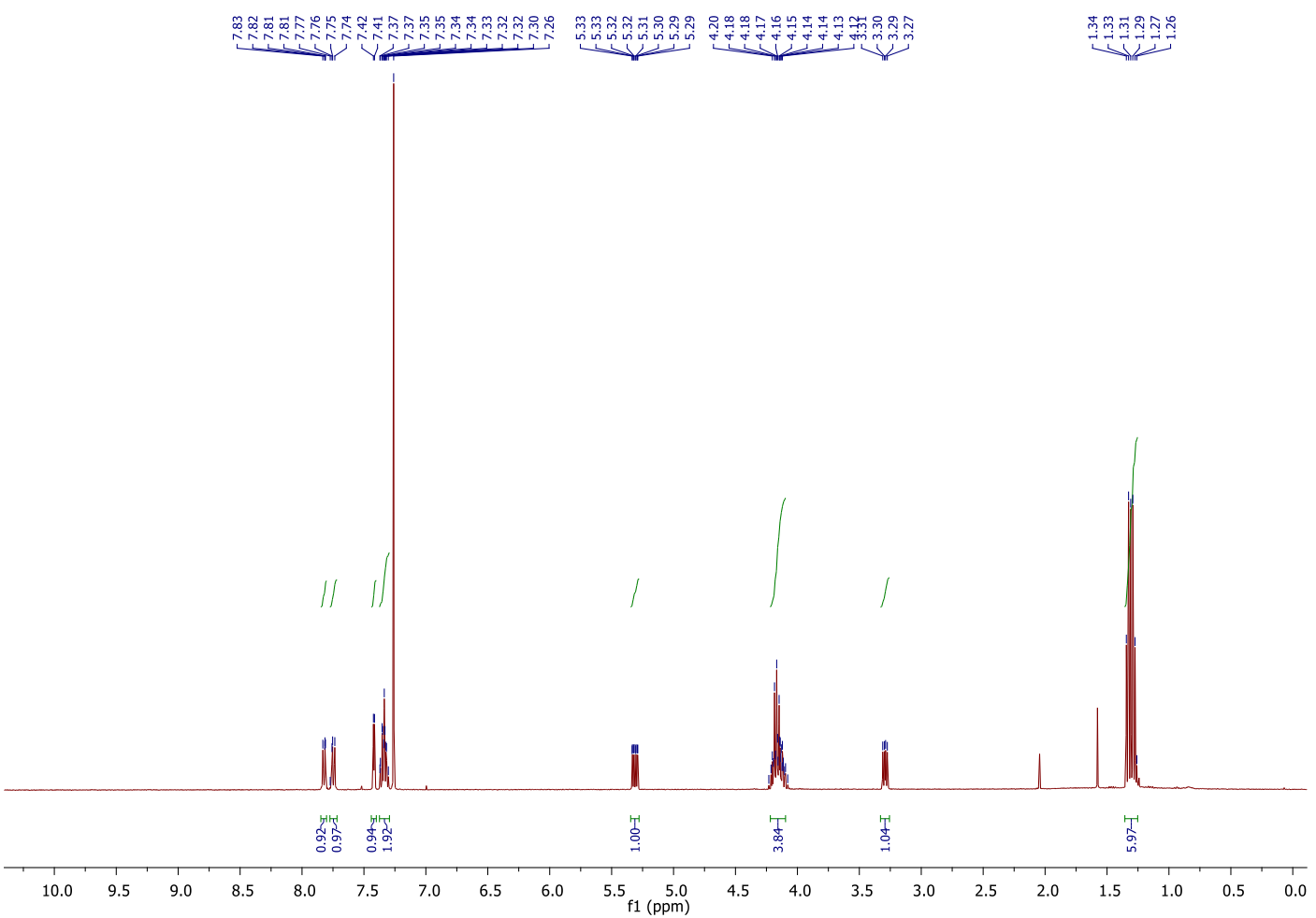

${ }^{13} \mathrm{C}-\mathrm{NMR}$ of compound $2 \mathrm{~m}\left(101 \mathrm{MHz}, \mathrm{CDCl}_{3}\right.$ )

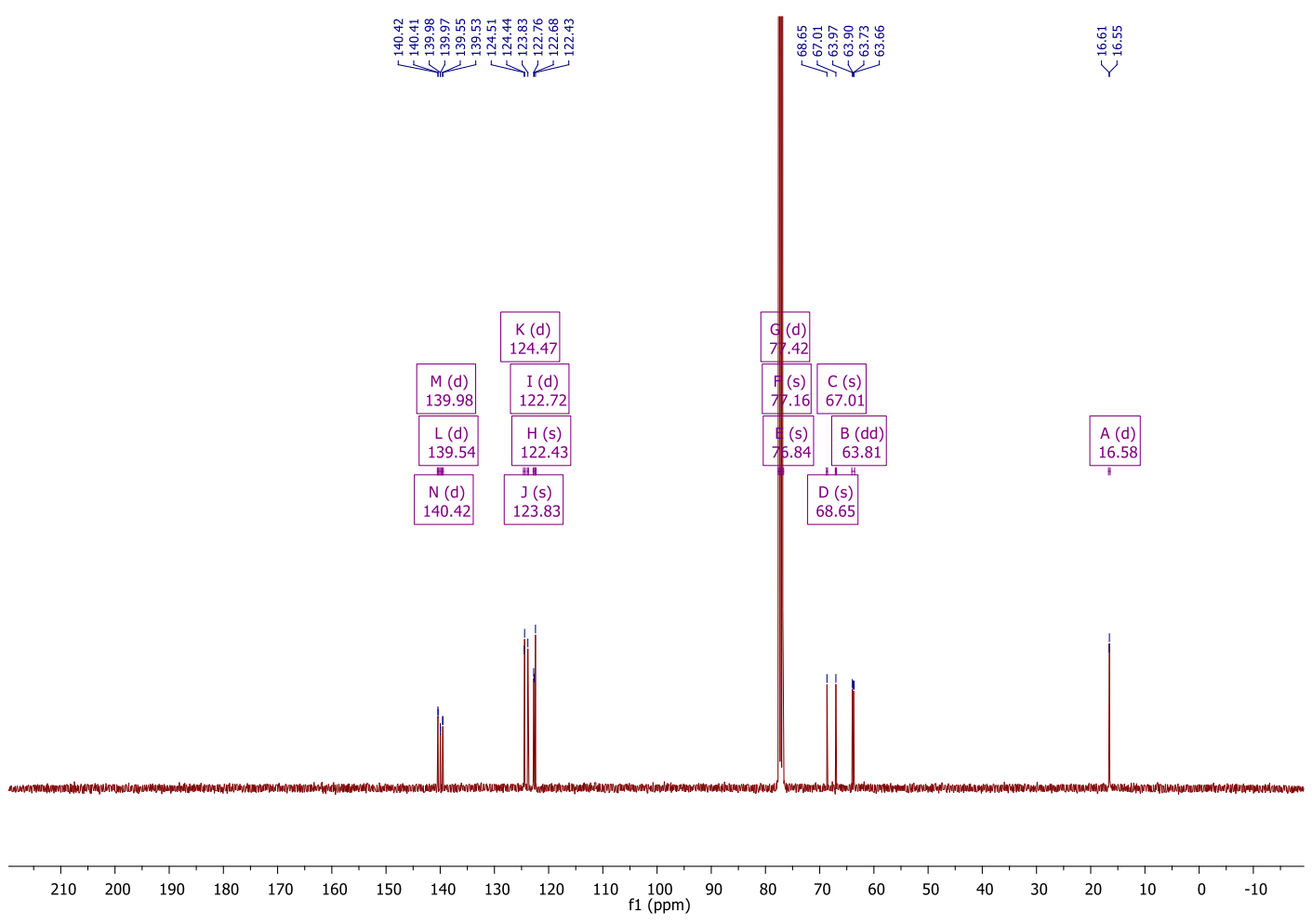


${ }^{1} \mathrm{H}-\mathrm{NMR}$ of compound $2 \mathrm{n}\left(400 \mathrm{MHz}^{\mathrm{CDCl}}\right)_{3}$

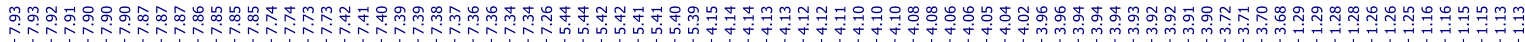

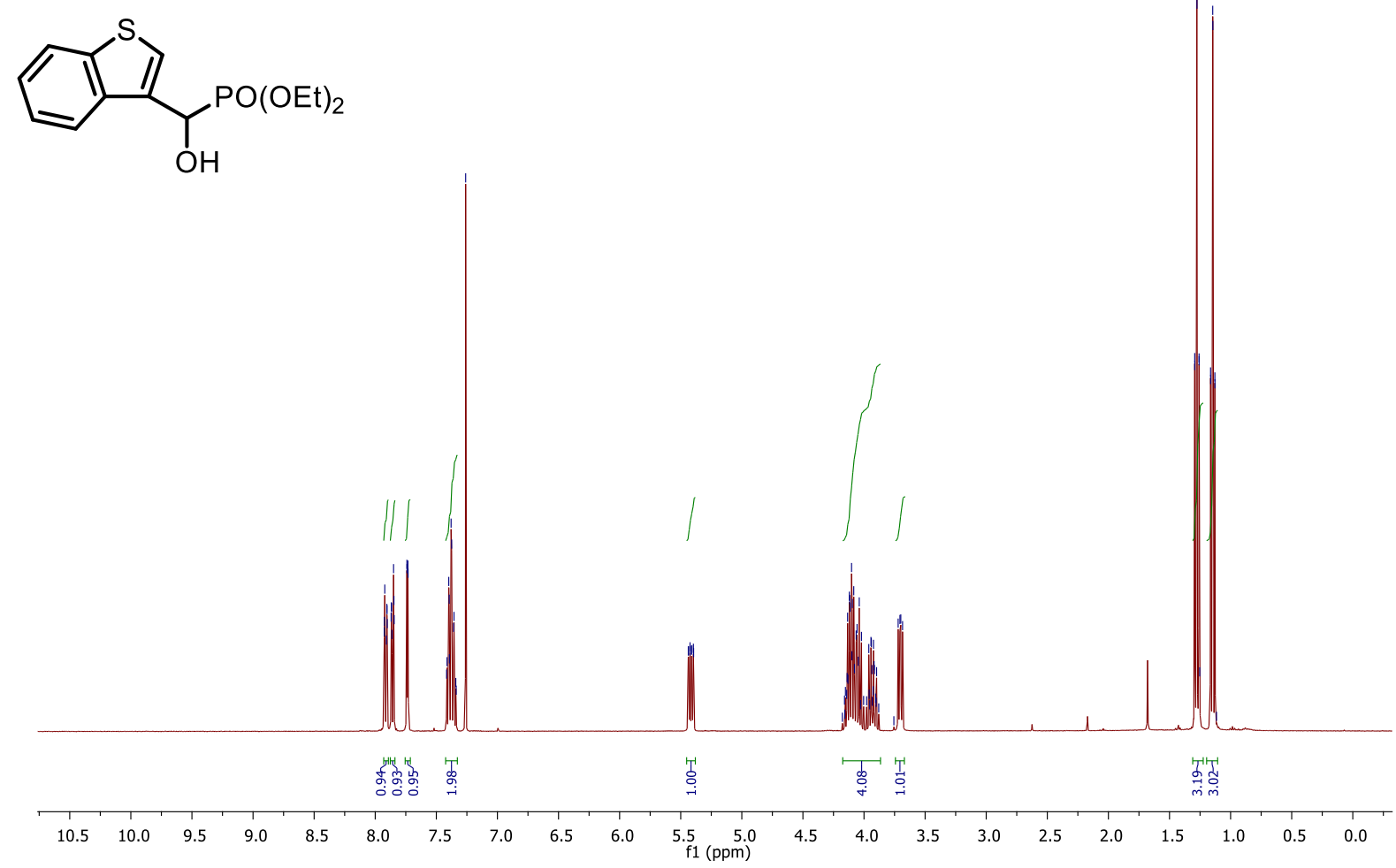

${ }^{13} \mathrm{C}-\mathrm{NMR}$ of compound $2 \mathrm{n}\left(101 \mathrm{MHz}, \mathrm{CDCl}_{3}\right)$

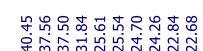

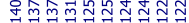

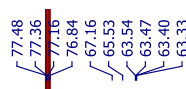

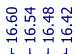

$|\vee| \forall \mid$

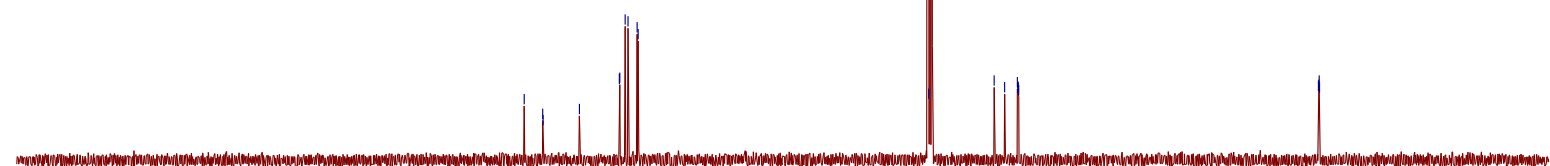

$\begin{array}{lllllllllllllllllllllllll}1 & 210 & 200 & 190 & 180 & 170 & 160 & 150 & 140 & 130 & 120 & 110 & \begin{array}{c}100 \\ \mathrm{f} 1(\mathrm{ppm})\end{array} & 90 & 80 & 70 & 60 & 50 & 40 & 30 & 20 & 10 & 0 & -10\end{array}$ 

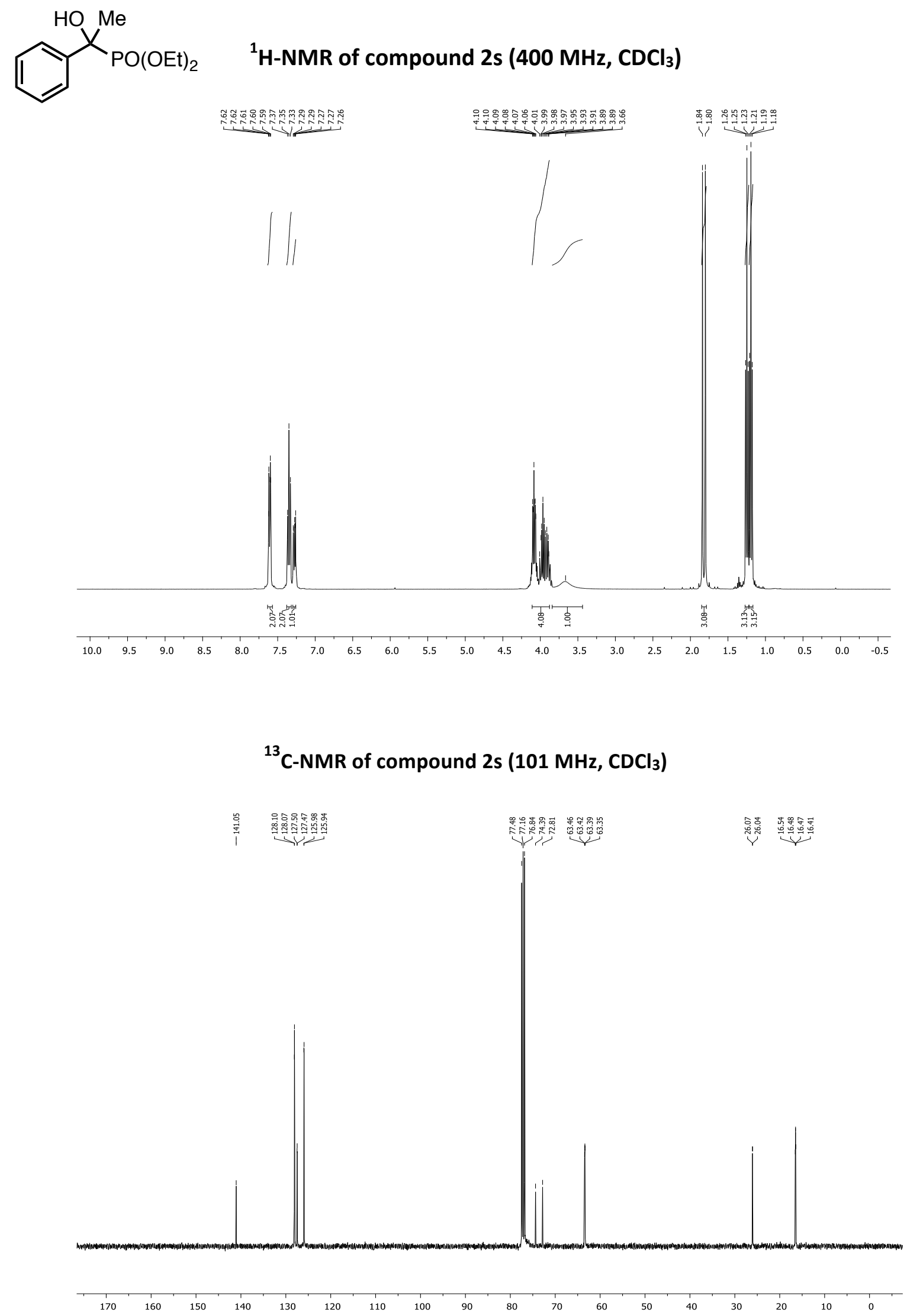


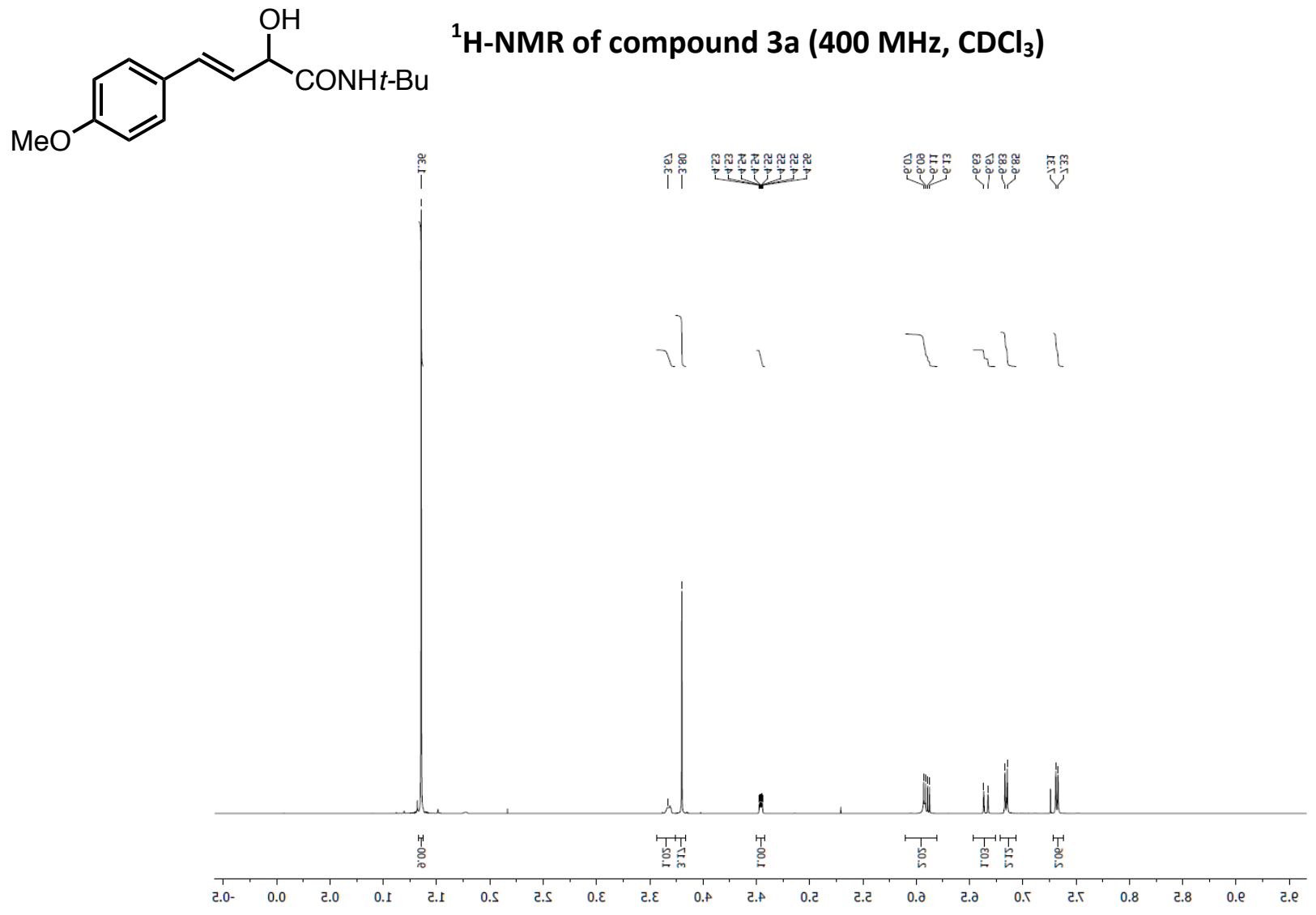

${ }^{13} \mathrm{C}-\mathrm{NMR}$ of compound 3a (101 $\mathrm{MHz}, \mathrm{CDCl}_{3}$ )

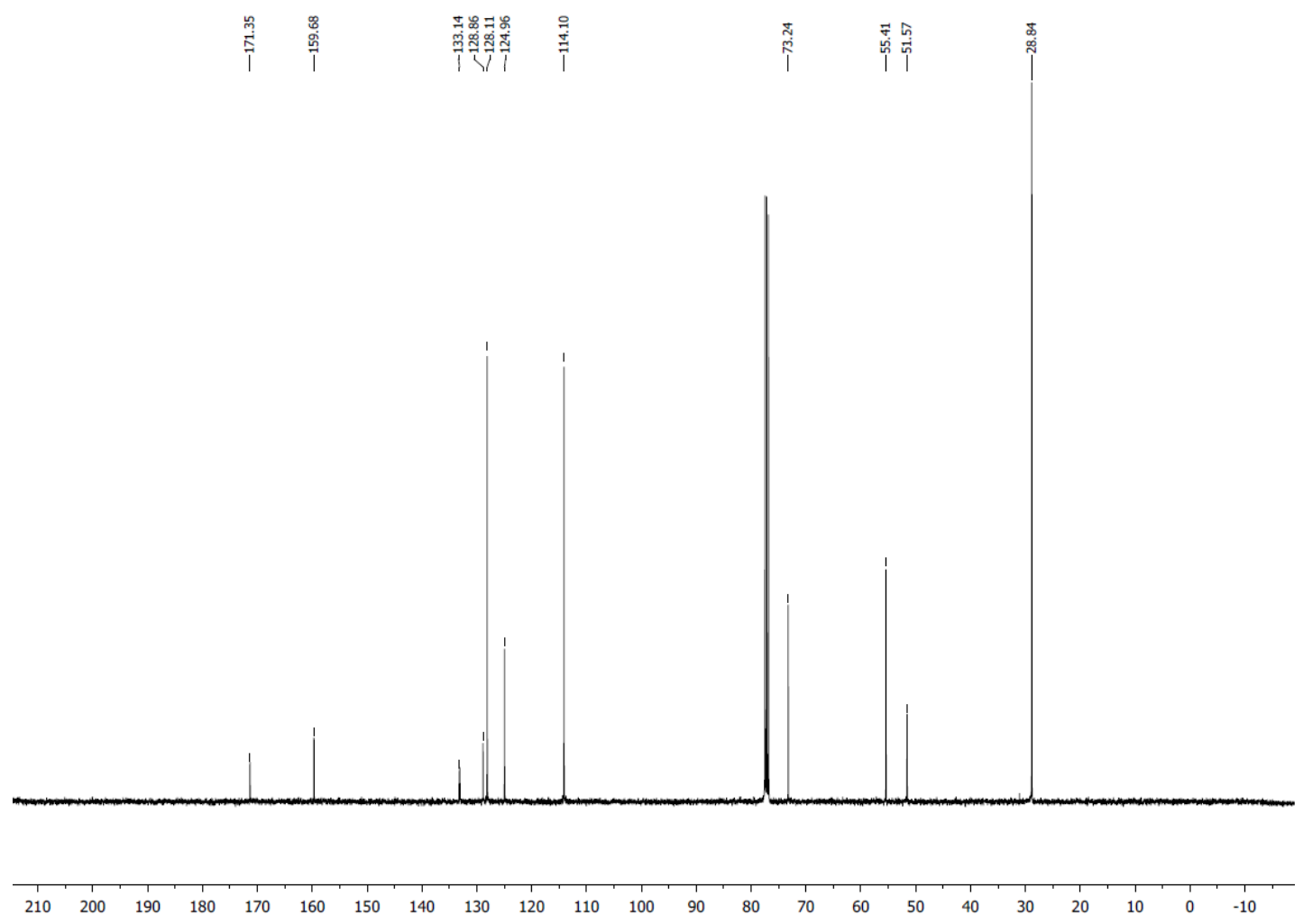


(1)

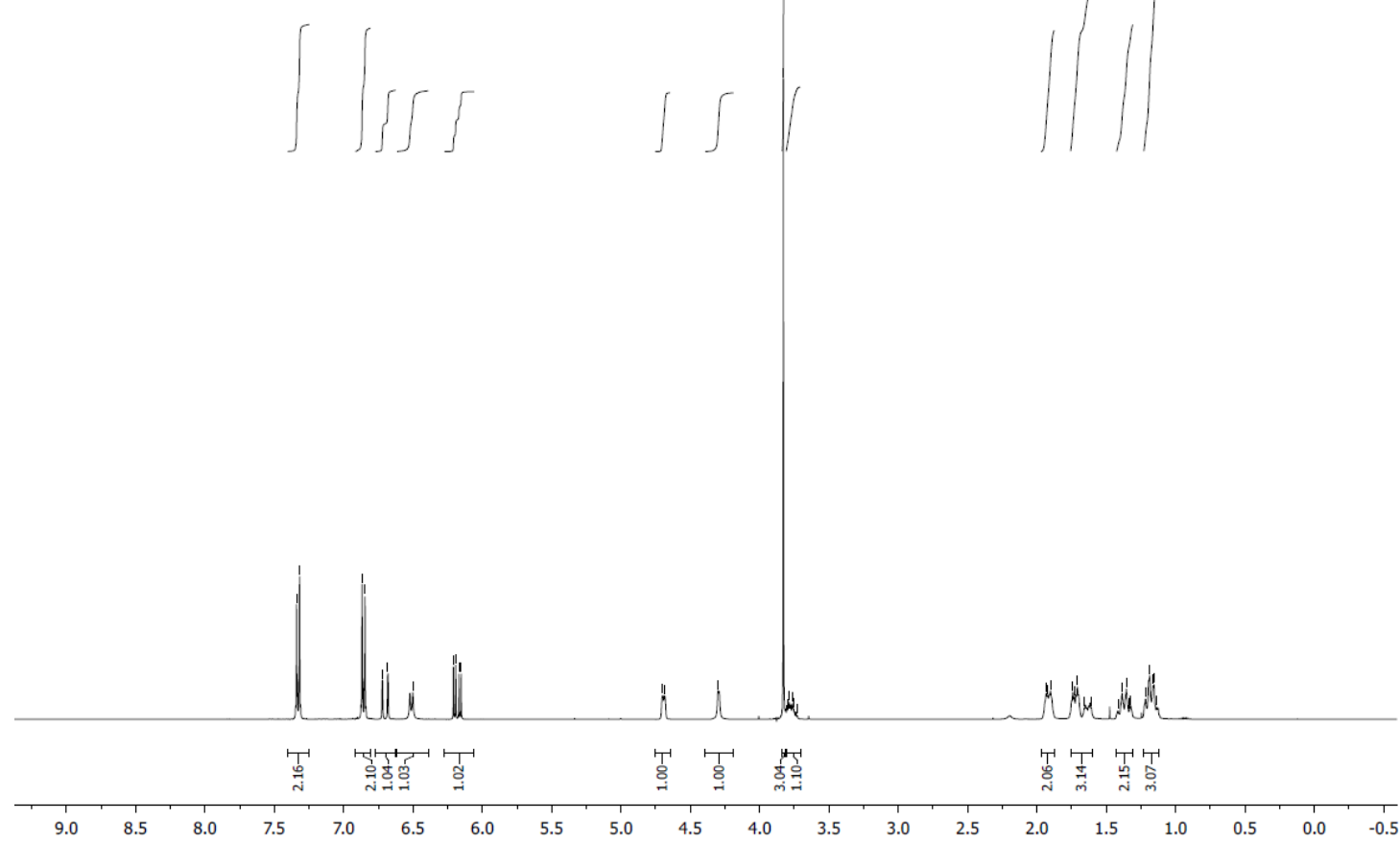

${ }^{13} \mathrm{C}-\mathrm{NMR}$ of compound $3 \mathrm{~b}\left(101 \mathrm{MHz}, \mathrm{CDCl}_{3}\right)$

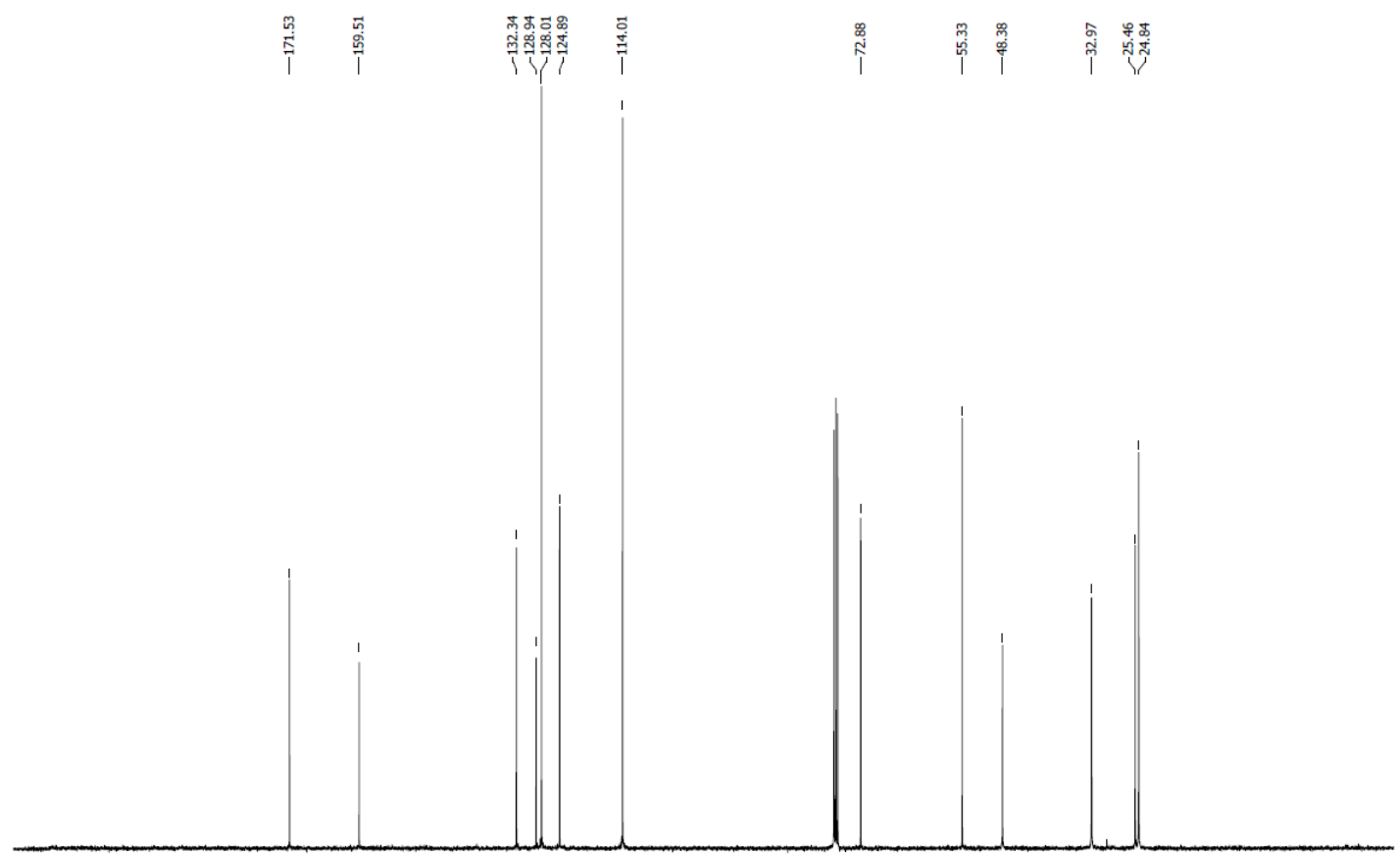

$\begin{array}{lllllllllllllllllllllll}210 & 200 & 190 & 180 & 170 & 160 & 150 & 140 & 130 & 120 & 110 & 100 & 90 & 80 & 70 & 60 & 50 & 40 & 30 & 20 & 10 & 0 & -10\end{array}$ 


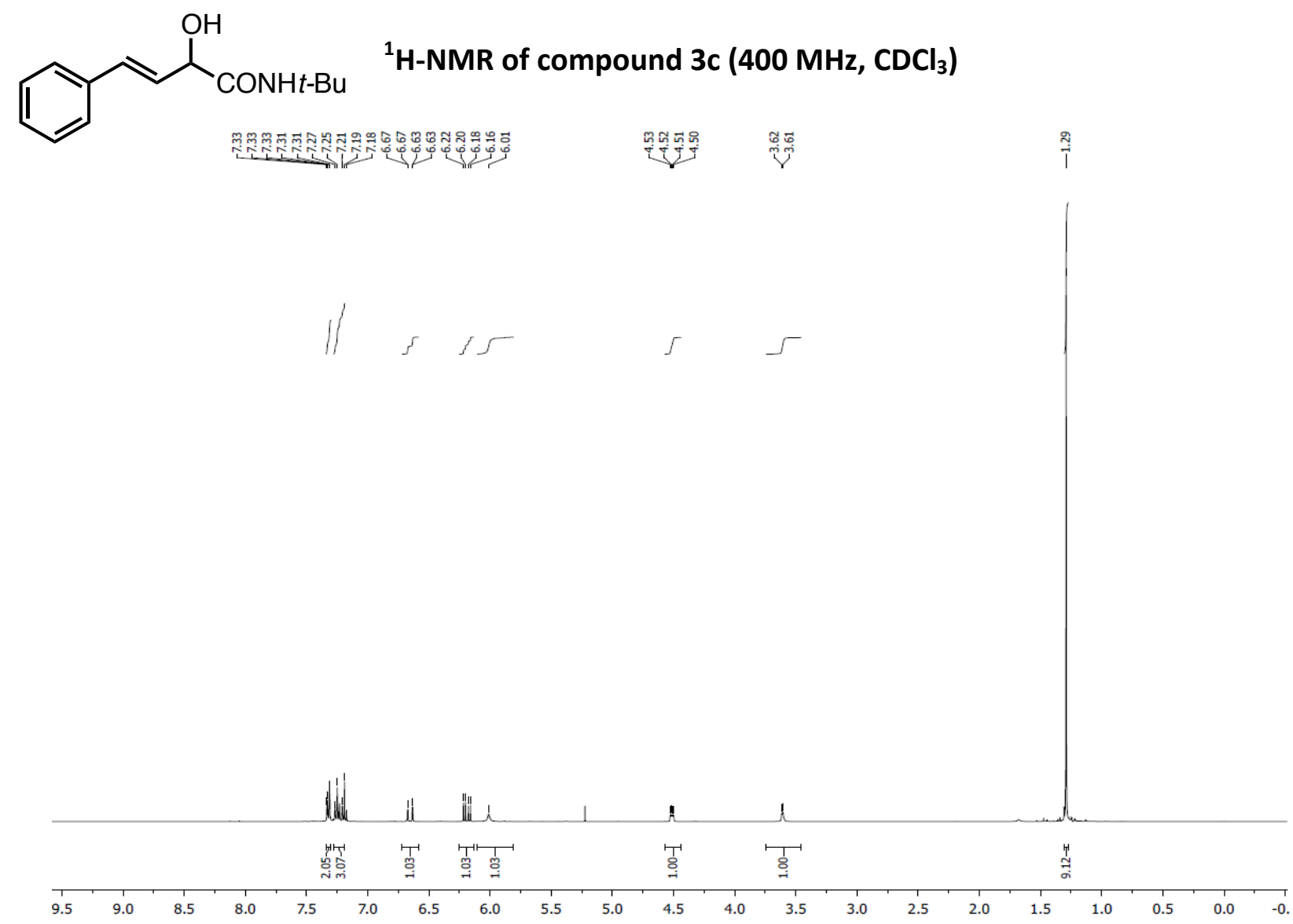

${ }^{13} \mathrm{C}-\mathrm{NMR}$ of compound 3c (101 $\left.\mathrm{MHz}, \mathrm{CDCl}_{3}\right)$

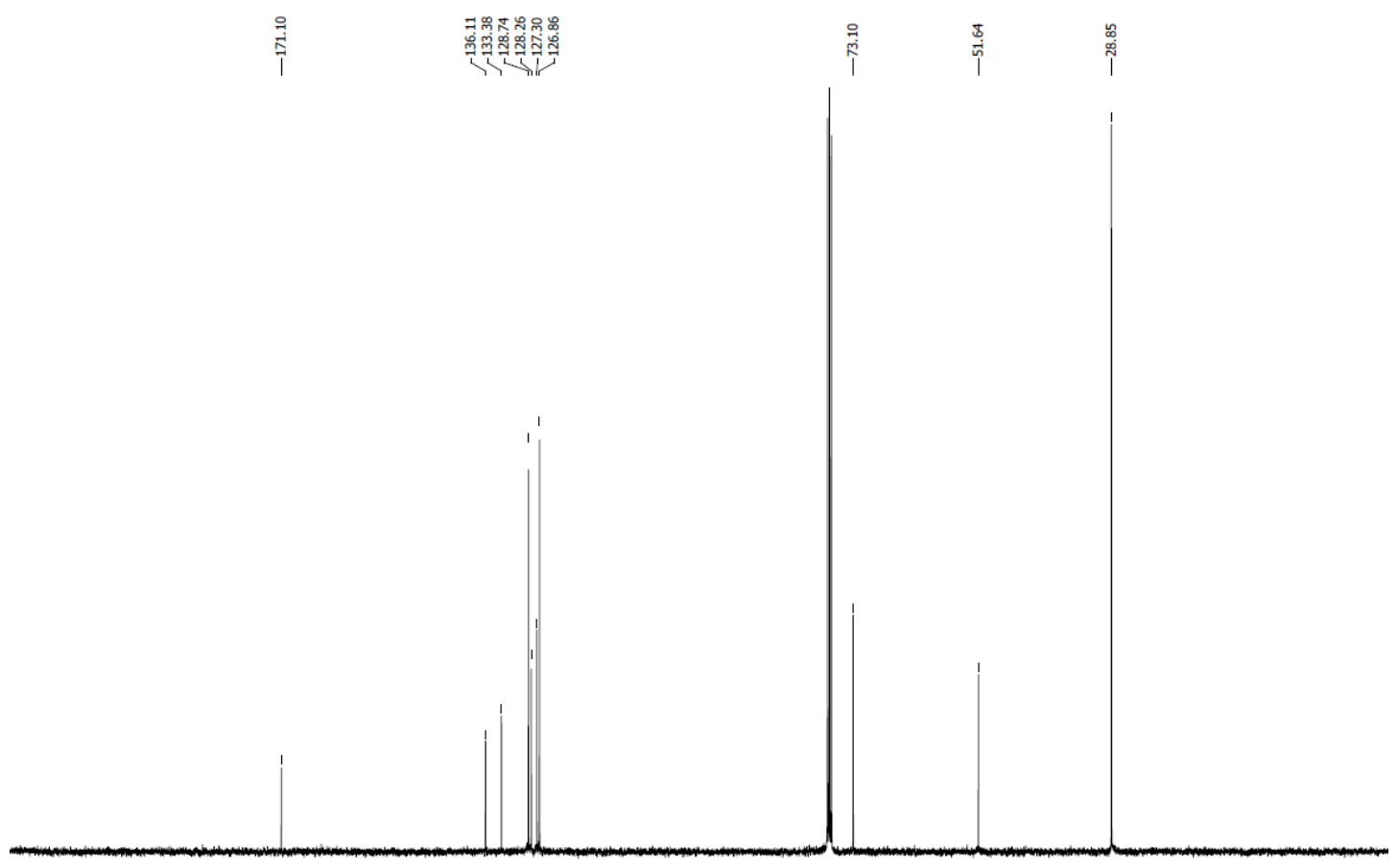

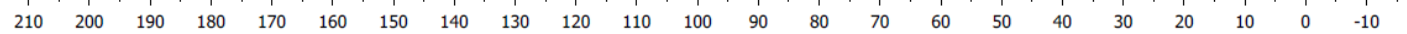




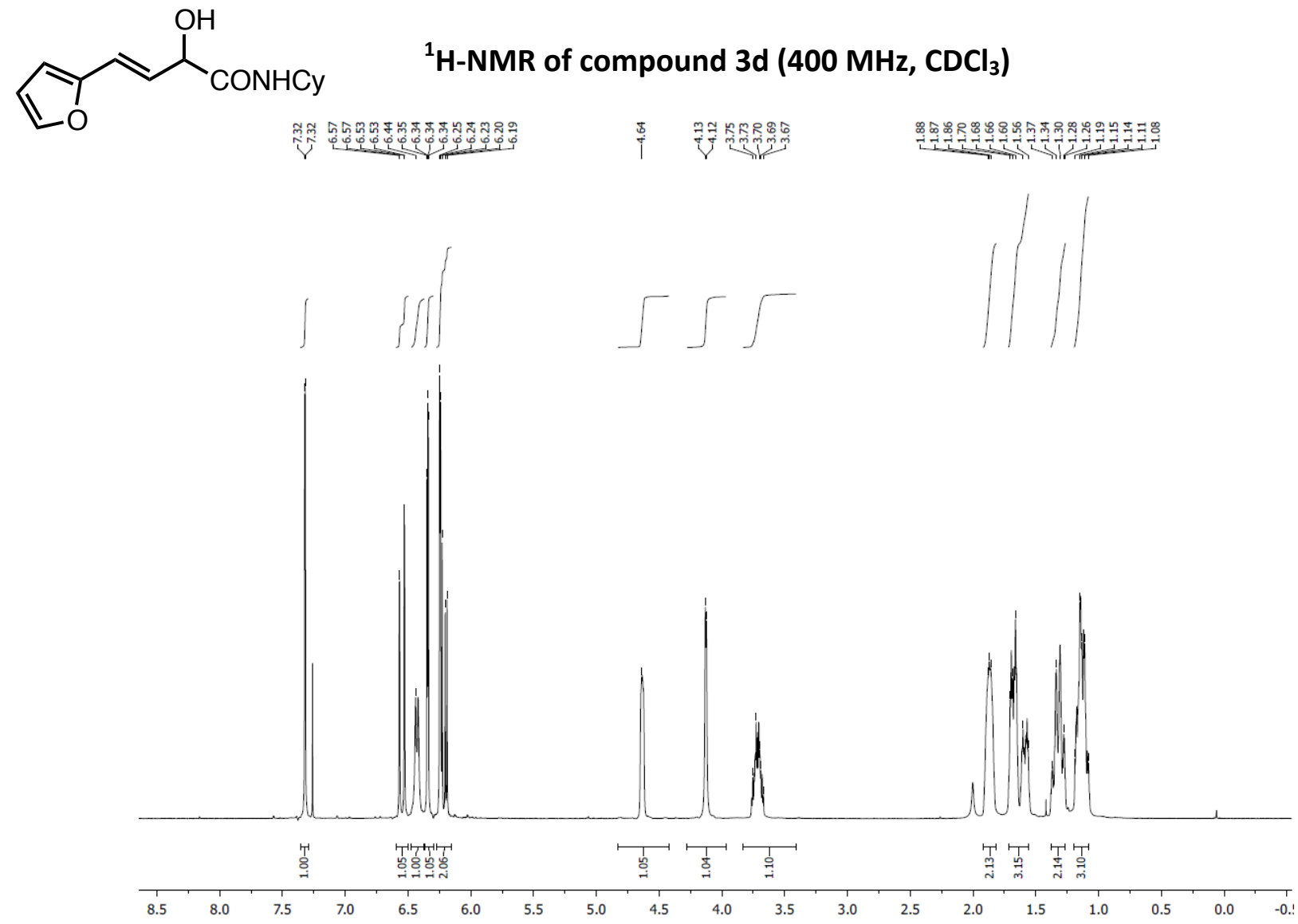

${ }^{13} \mathrm{C}-\mathrm{NMR}$ of compound 3d (101 $\left.\mathrm{MHz}, \mathrm{CDCl}_{3}\right)$

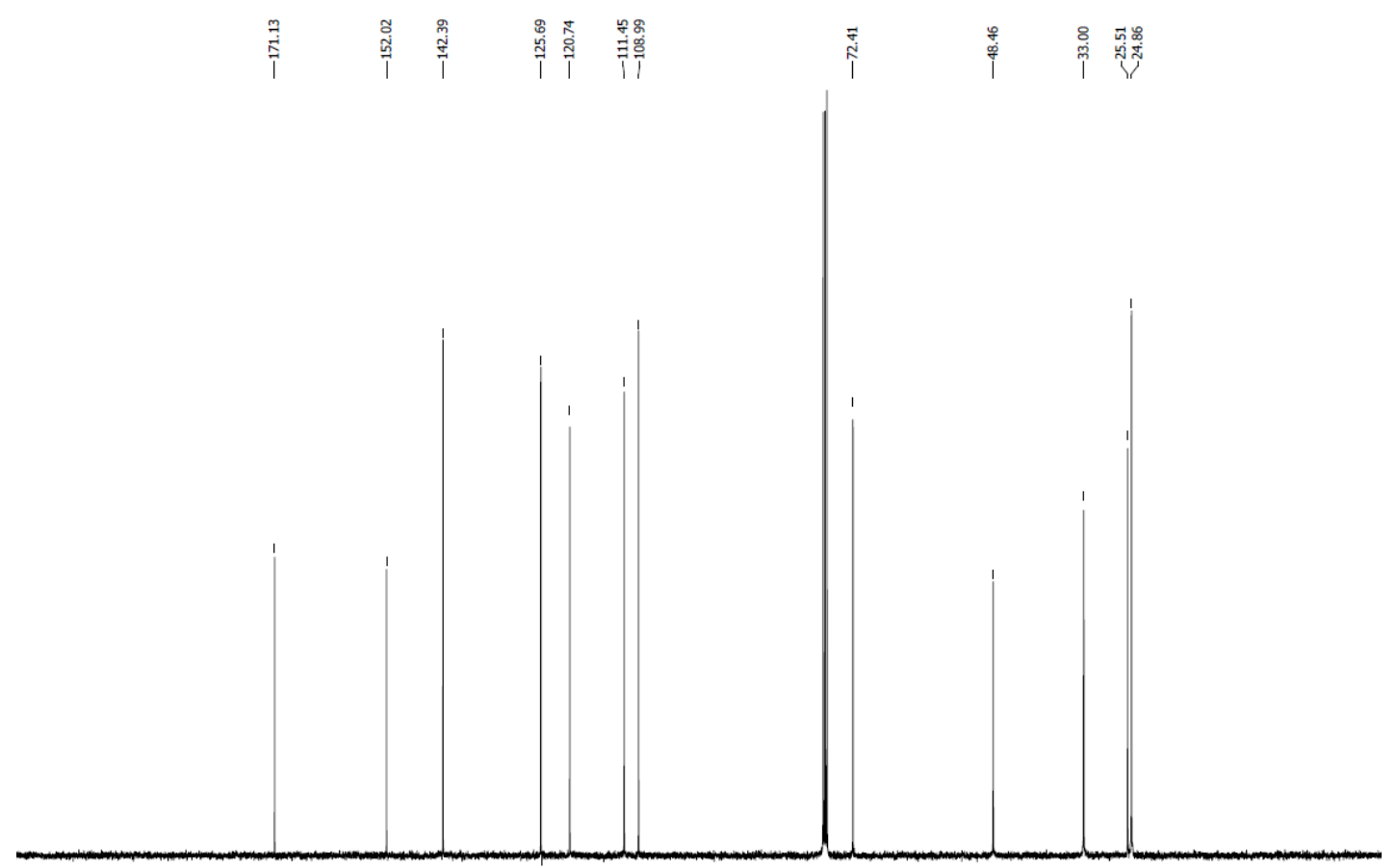

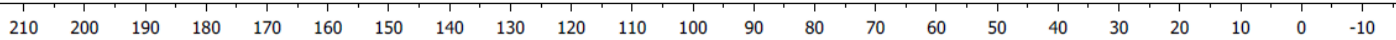




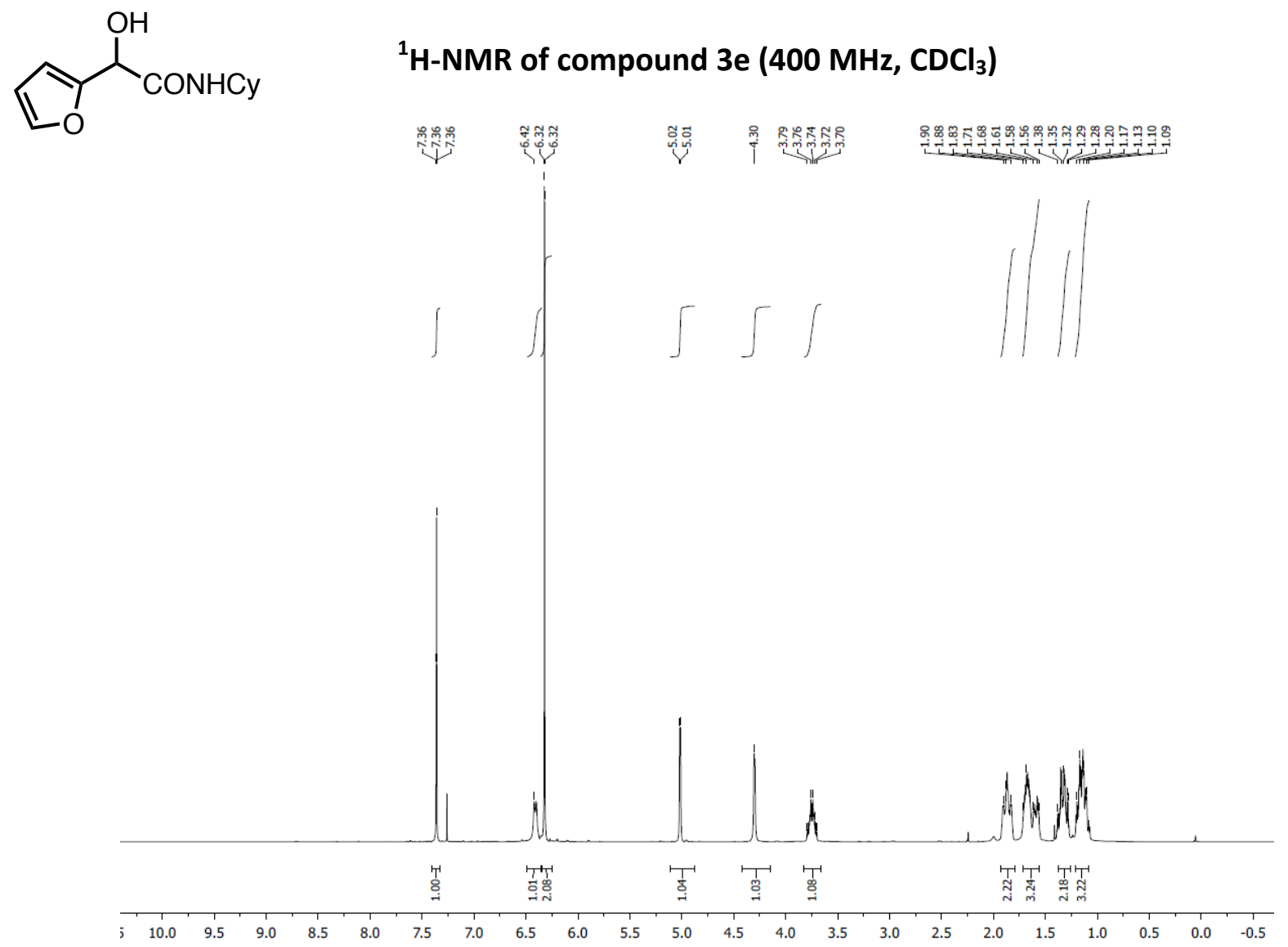

${ }^{13} \mathrm{C}-\mathrm{NMR}$ of compound $3 \mathrm{e}\left(101 \mathrm{MHz}, \mathrm{CDCl}_{3}\right)$

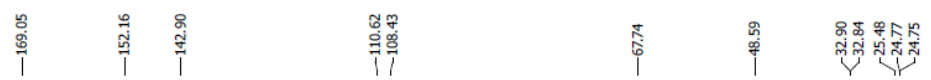

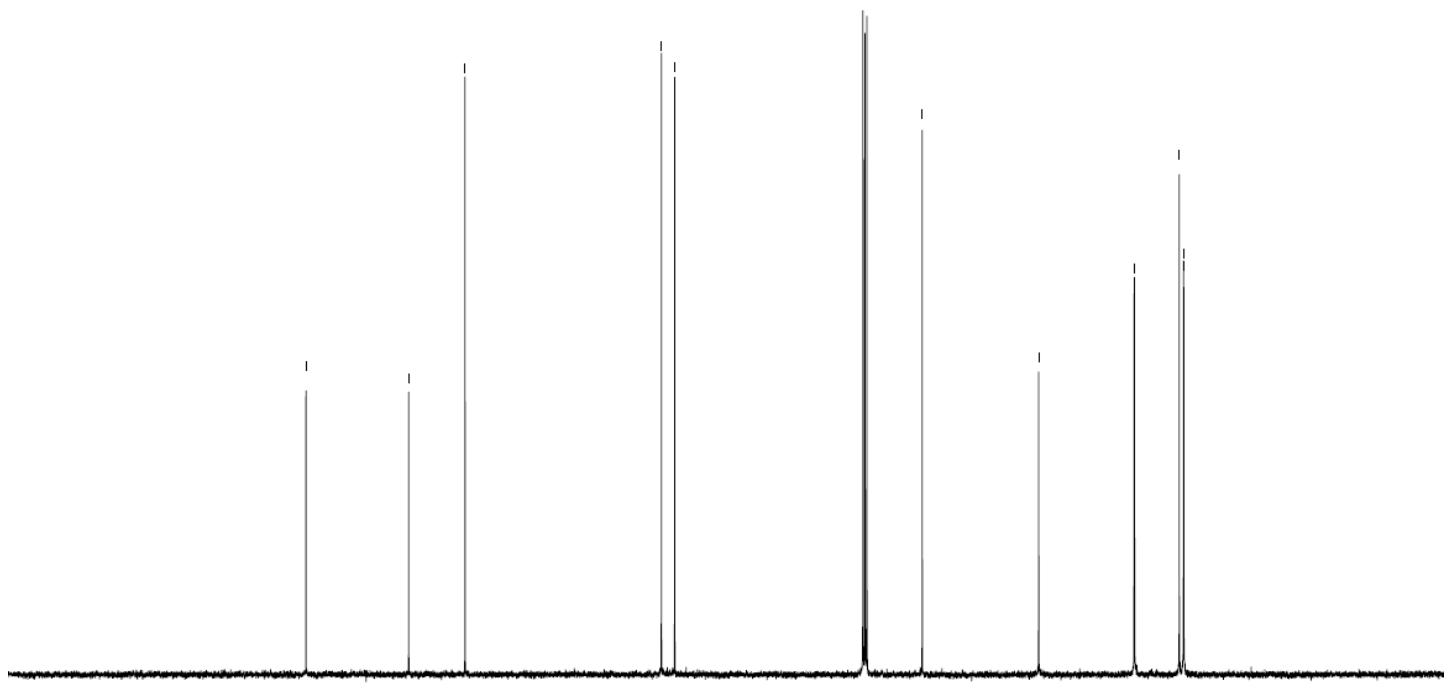

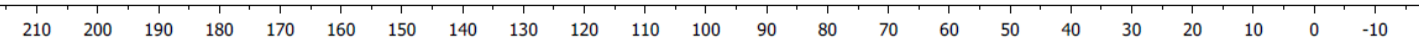


${ }^{1} \mathrm{H}-\mathrm{NMR}$ of compound $5 \mathrm{j}\left(400 \mathrm{MHz}, \mathrm{CDCl}_{3}\right)$

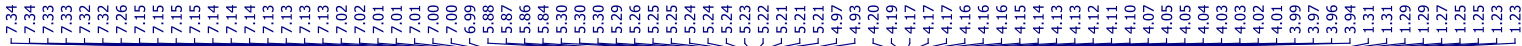<smiles>C=CCOC(OCC)c1cccs1</smiles>

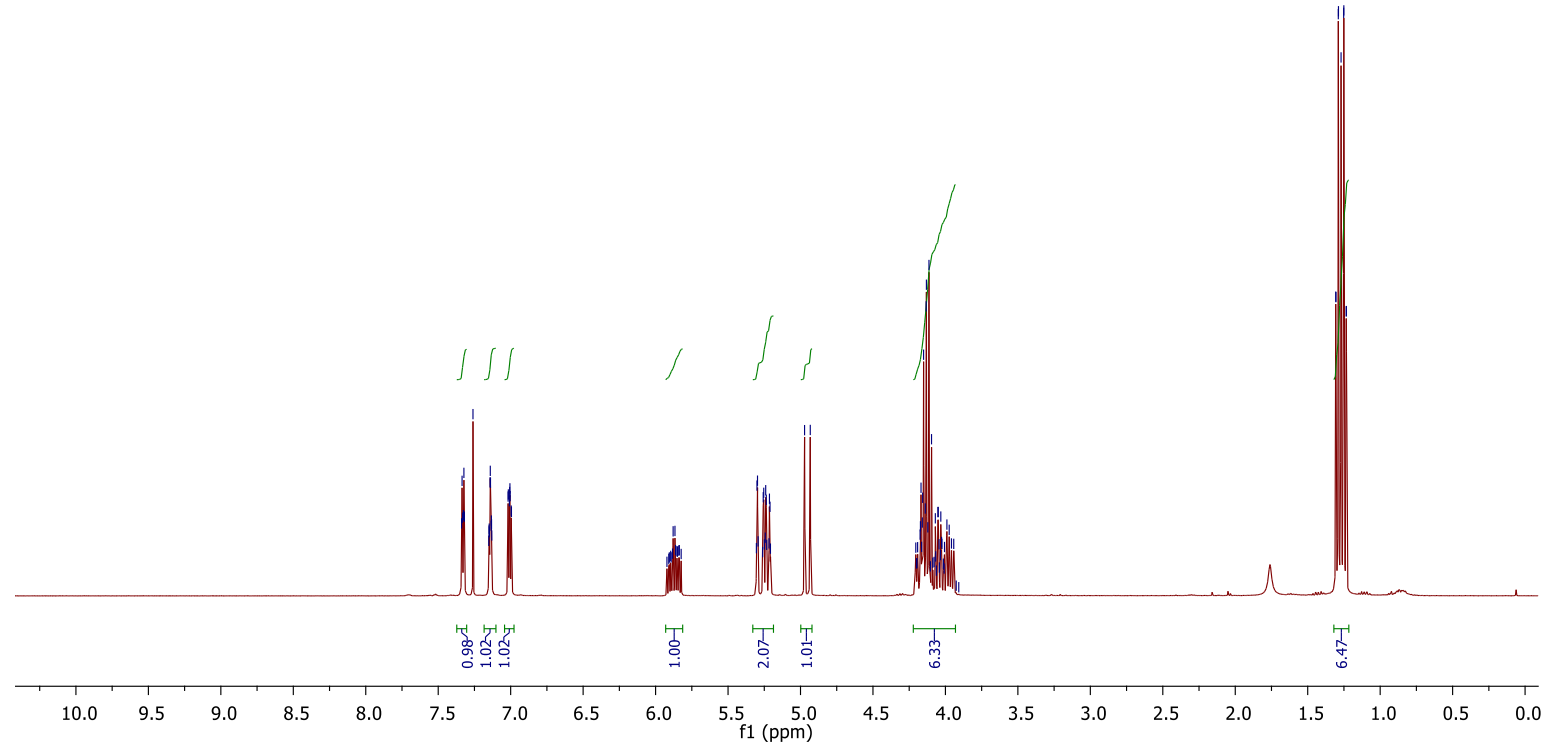

${ }^{13} \mathrm{C}-\mathrm{NMR}$ of compound $5 \mathrm{j}\left(101 \mathrm{MHz}, \mathrm{CDCl}_{3}\right)$

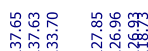

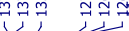

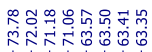

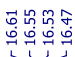

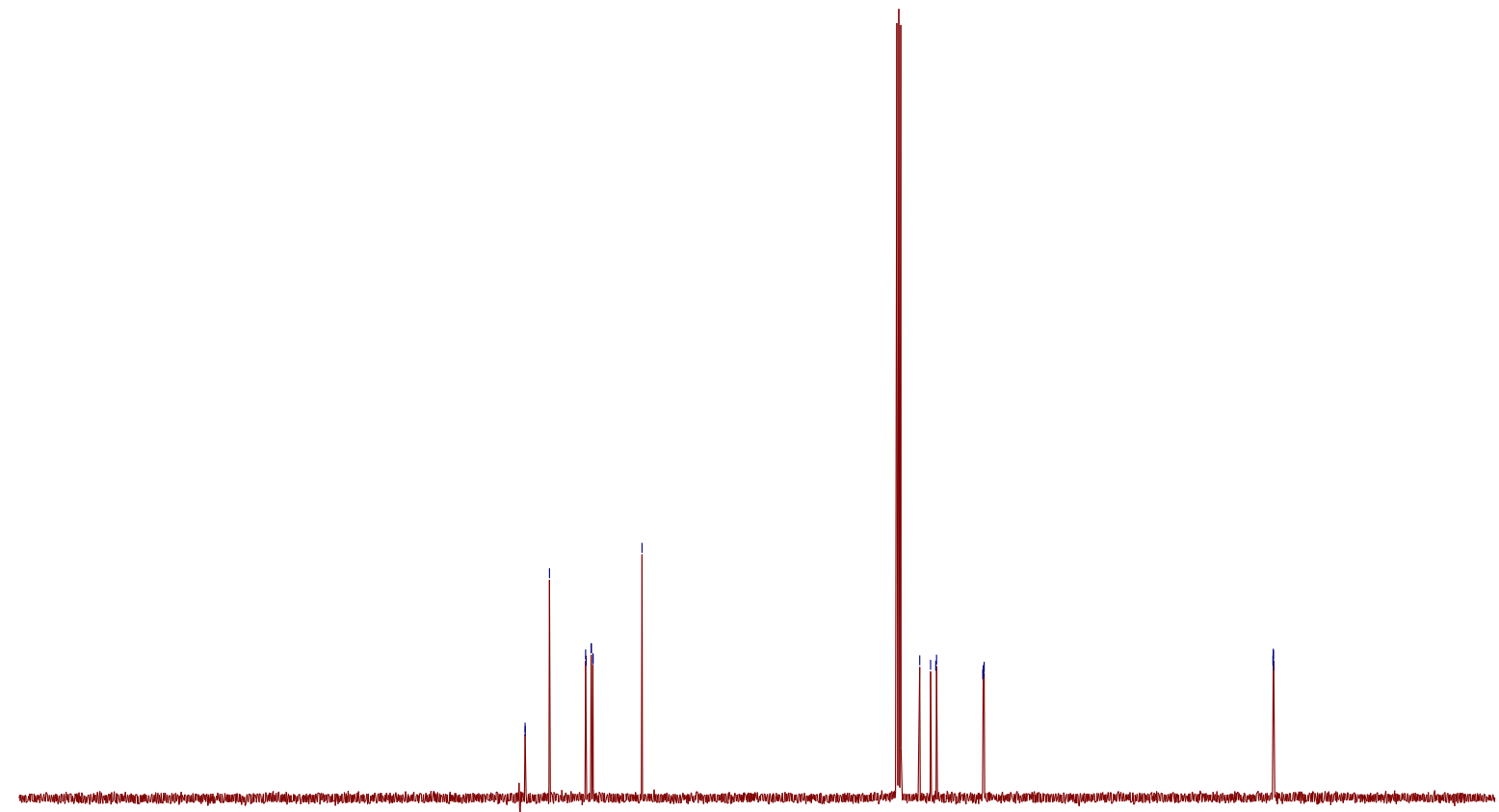

$\begin{array}{llllllllllllllllllllllllllllll}210 & 200 & 190 & 180 & 170 & 160 & 150 & 140 & 130 & 120 & 110 & 100 & 90 & 80 & 70 & 60 & 50 & 40 & 30 & 20 & 10 & 0 & -10\end{array}$ 


\section{${ }^{1} \mathrm{H}-\mathrm{NMR}$ of compound $5 \mathrm{k}\left(400 \mathrm{MHz}, \mathrm{CDCl}_{3}\right)$}

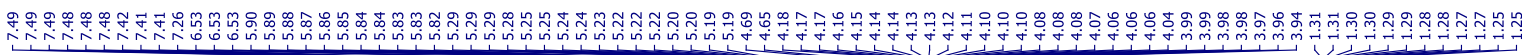<smiles>C=CCOC(POCC)c1ccoc1</smiles>

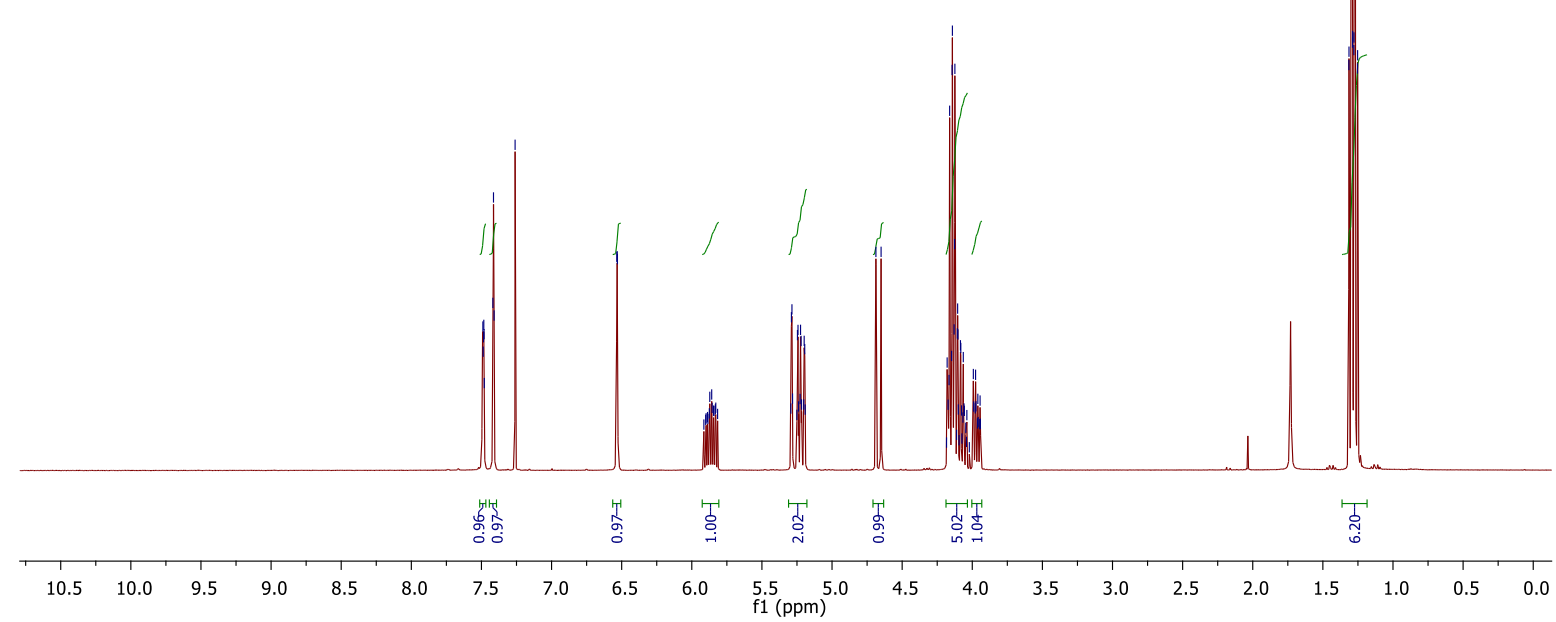

${ }^{13} \mathrm{C}-\mathrm{NMR}$ of compound $5 \mathrm{k}\left(101 \mathrm{MHz}, \mathrm{CDCl}_{3}\right)$

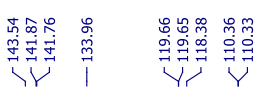

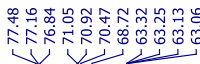

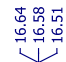

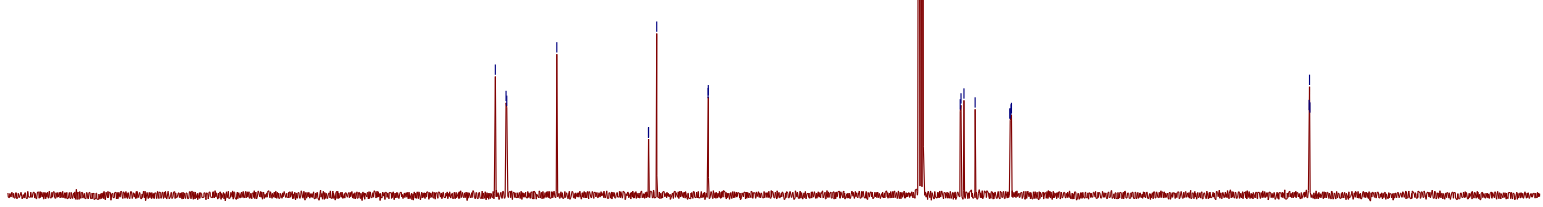

$\begin{array}{lllllllllllllllllllllllll}210 & 200 & 190 & 180 & 170 & 160 & 150 & 140 & 130 & 120 & 110 & 100 & 90 & 80 & 70 & 60 & 50 & 40 & 30 & 20 & 10 & 0 & -10\end{array}$ 
${ }^{1} \mathrm{H}$-NMR of compound $5 \mathrm{I}\left(400 \mathrm{MHz}^{\mathrm{CDCl}}{ }_{3}\right.$ )

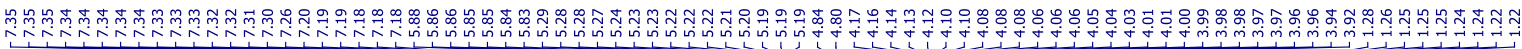<smiles>C=CCOC(POCC)c1ccsc1</smiles>

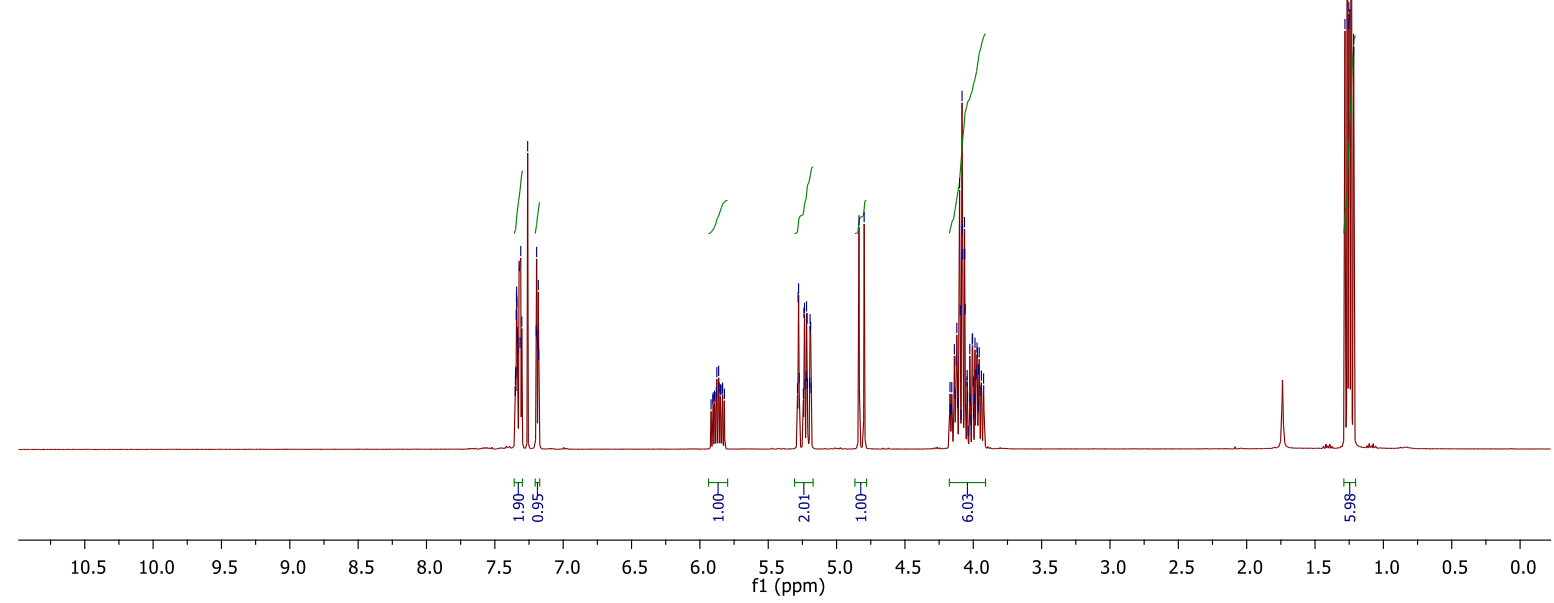

${ }^{13} \mathrm{C}$-NMR of compound $5 \mathrm{I}\left(101 \mathrm{MHz}, \mathrm{CDCl}_{3}\right.$ )

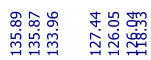

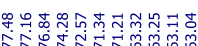

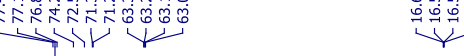

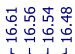

Y) if

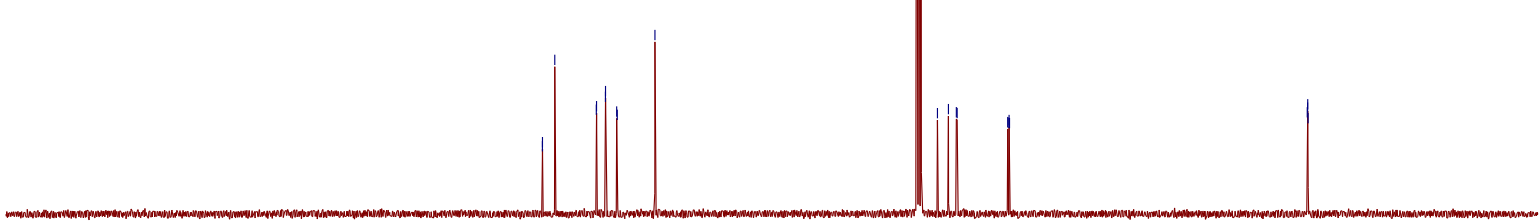

$\begin{array}{lllllllllllllllllllllll}210 & 200 & 190 & 180 & 170 & 160 & 150 & 140 & 130 & 120 & 110 & \begin{array}{c}100 \\ \mathrm{f} 1(\mathrm{ppm})\end{array} & 90 & 80 & 70 & 60 & 50 & 40 & 30 & 20 & 10 & 0 & -10\end{array}$ 
<smiles>C=CCOC(POCC)c1cc2ccccc2s1</smiles>

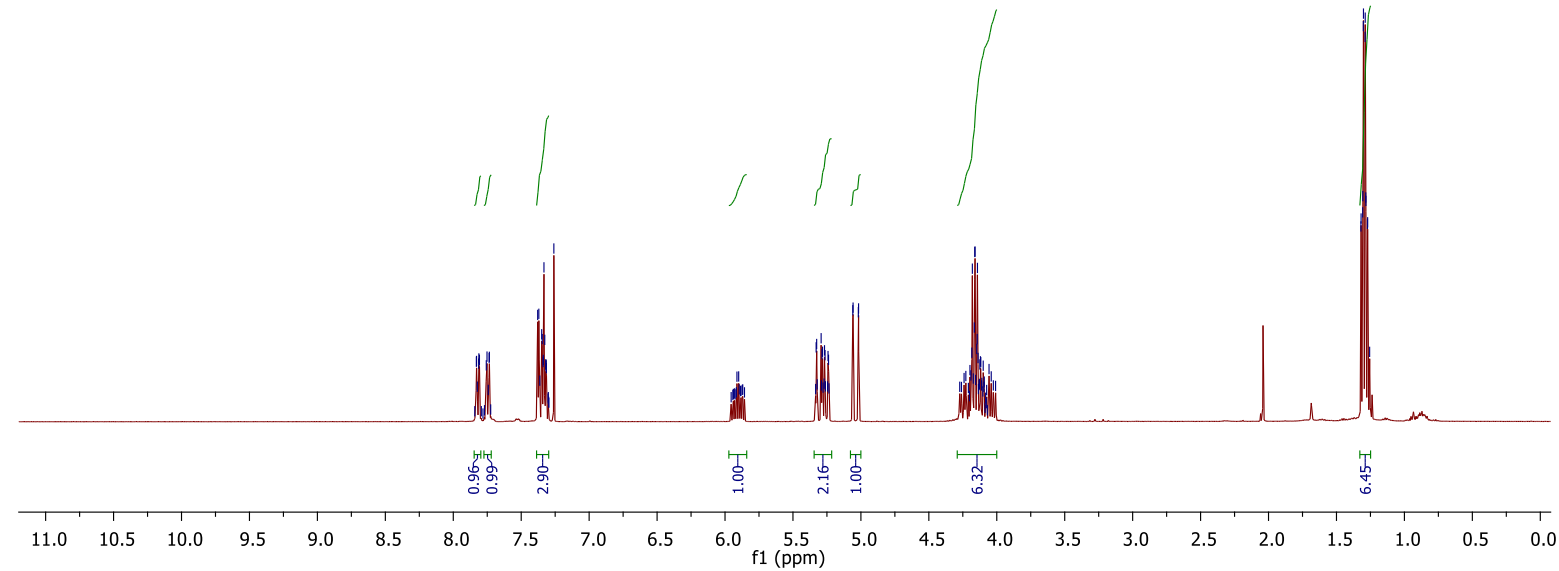

${ }^{13} \mathrm{C}-\mathrm{NMR}$ of compound $5 \mathrm{~m}\left(101 \mathrm{MHz}, \mathrm{CDCl}_{3}\right)$

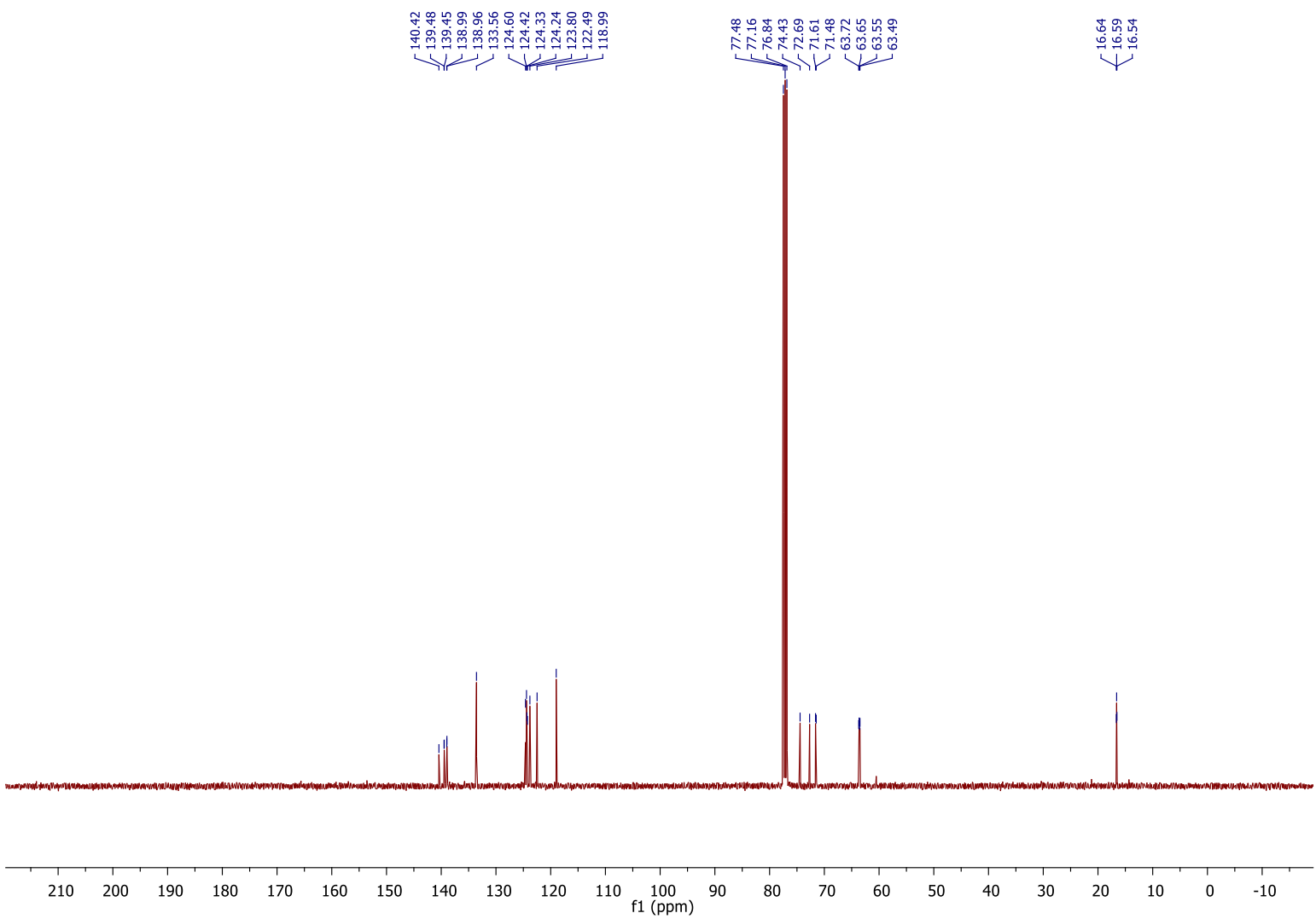


<smiles>C=CCOC(POCC)c1csc2ccccc12</smiles>

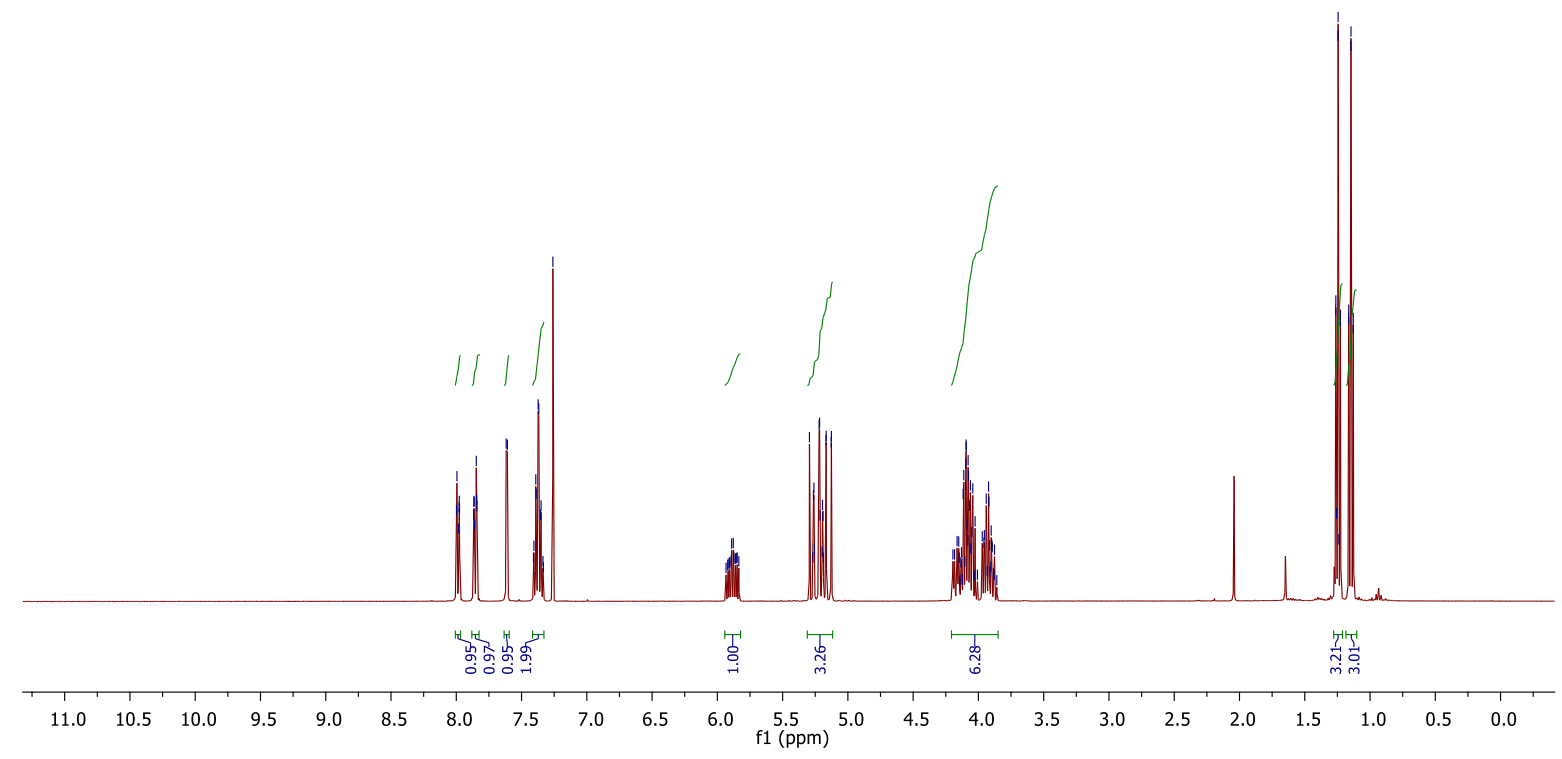

${ }^{13} \mathrm{C}-\mathrm{NMR}$ of compound $5 \mathrm{n}\left(101 \mathrm{MHz}, \mathrm{CDCl}_{3}\right)$

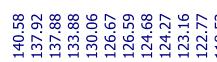

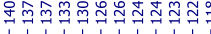

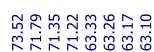

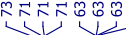

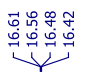

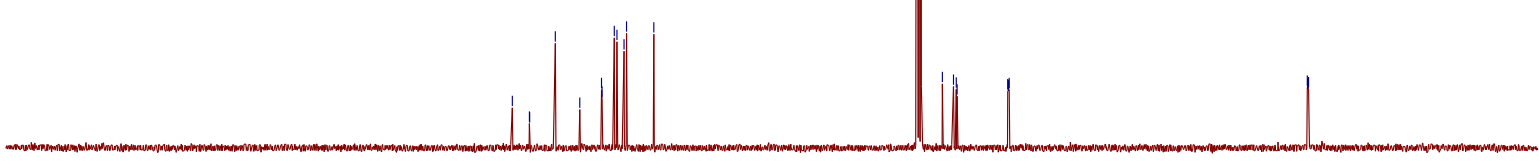

$\begin{array}{lllllllllllllllllllllll}210 & 200 & 190 & 180 & 170 & 160 & 150 & 140 & 130 & 120 & 110 \underset{\mathrm{f} 1(\mathrm{ppm})}{100} & 90 & 80 & 70 & 60 & 50 & 40 & 30 & 20 & 10 & 0 & -10\end{array}$ 


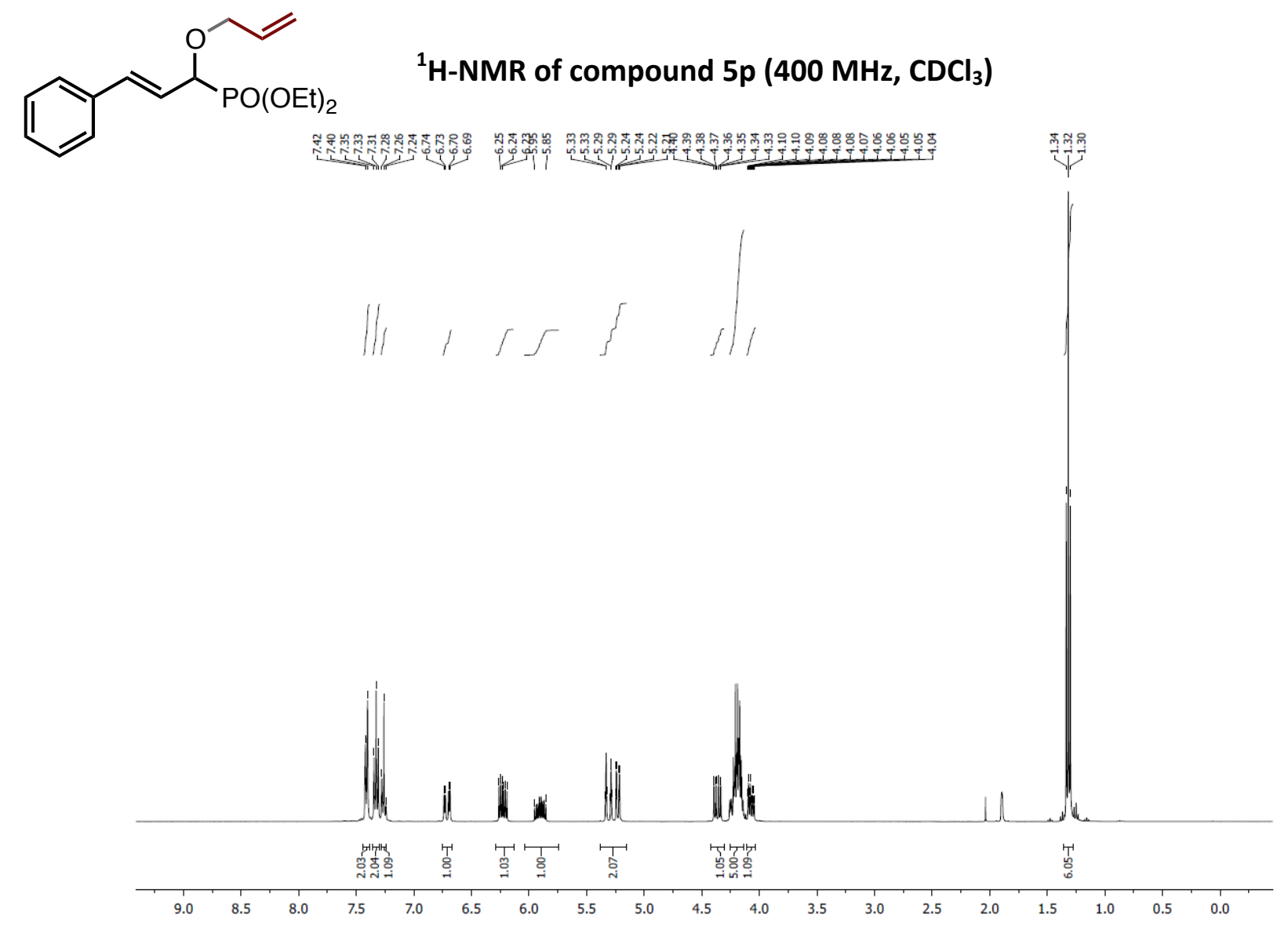

${ }^{13} \mathrm{C}-\mathrm{NMR}$ of compound $5 \mathrm{p}\left(101 \mathrm{MHz}, \mathrm{CDCl}_{3}\right)$

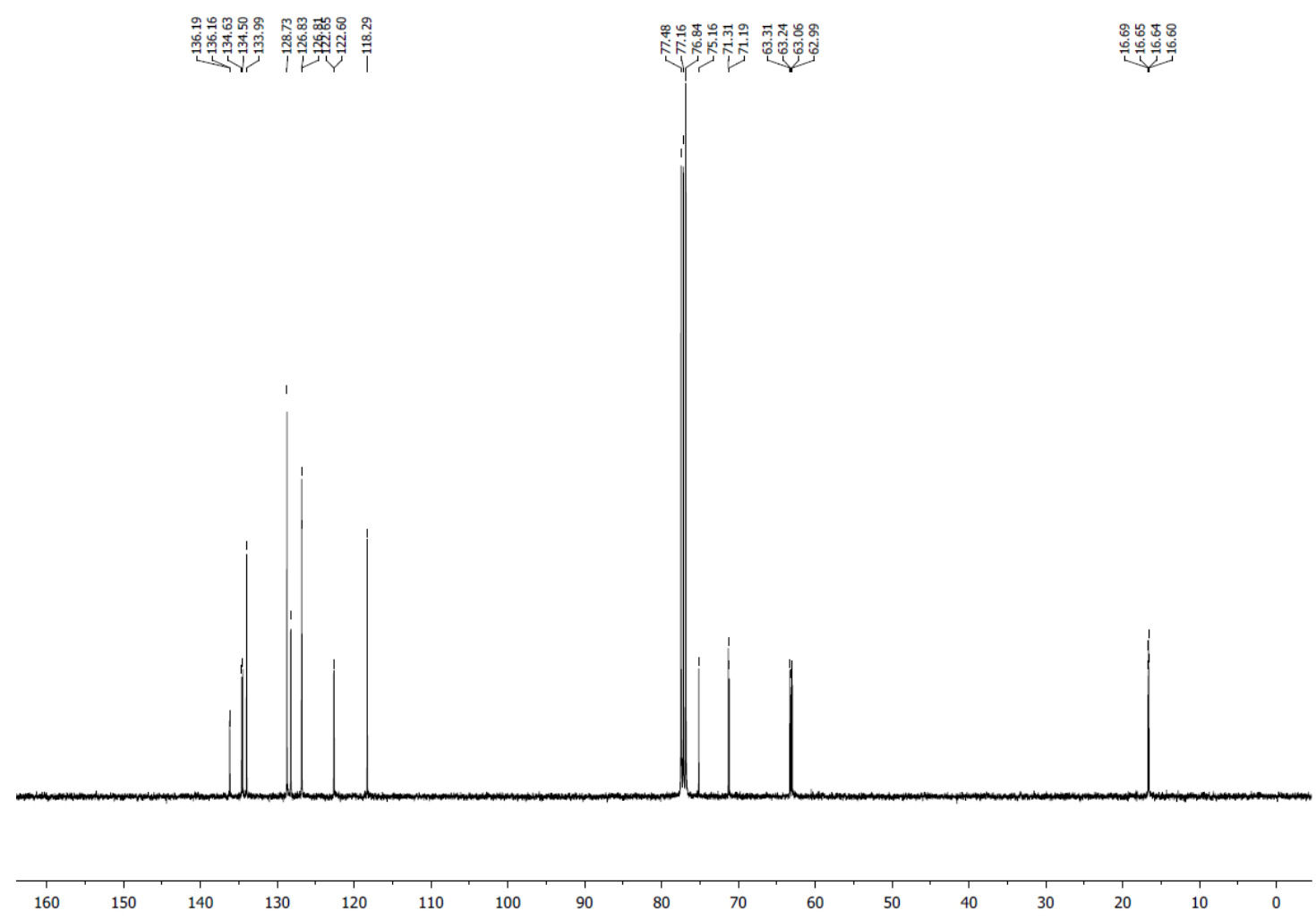




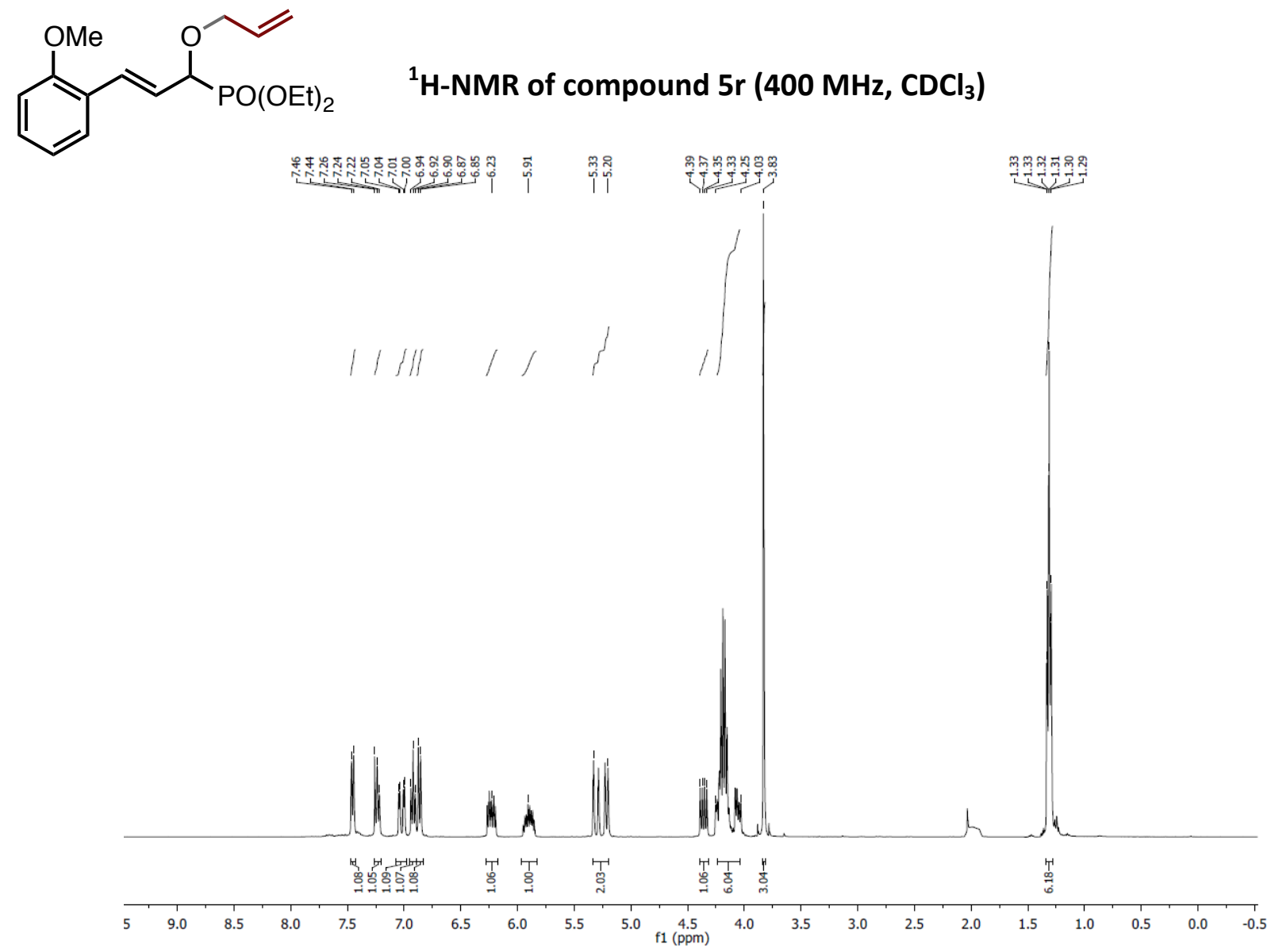

${ }^{13} \mathrm{C}-\mathrm{NMR}$ of compound $5 \mathrm{r}\left(101 \mathrm{MHz}, \mathrm{CDCl}_{3}\right)$

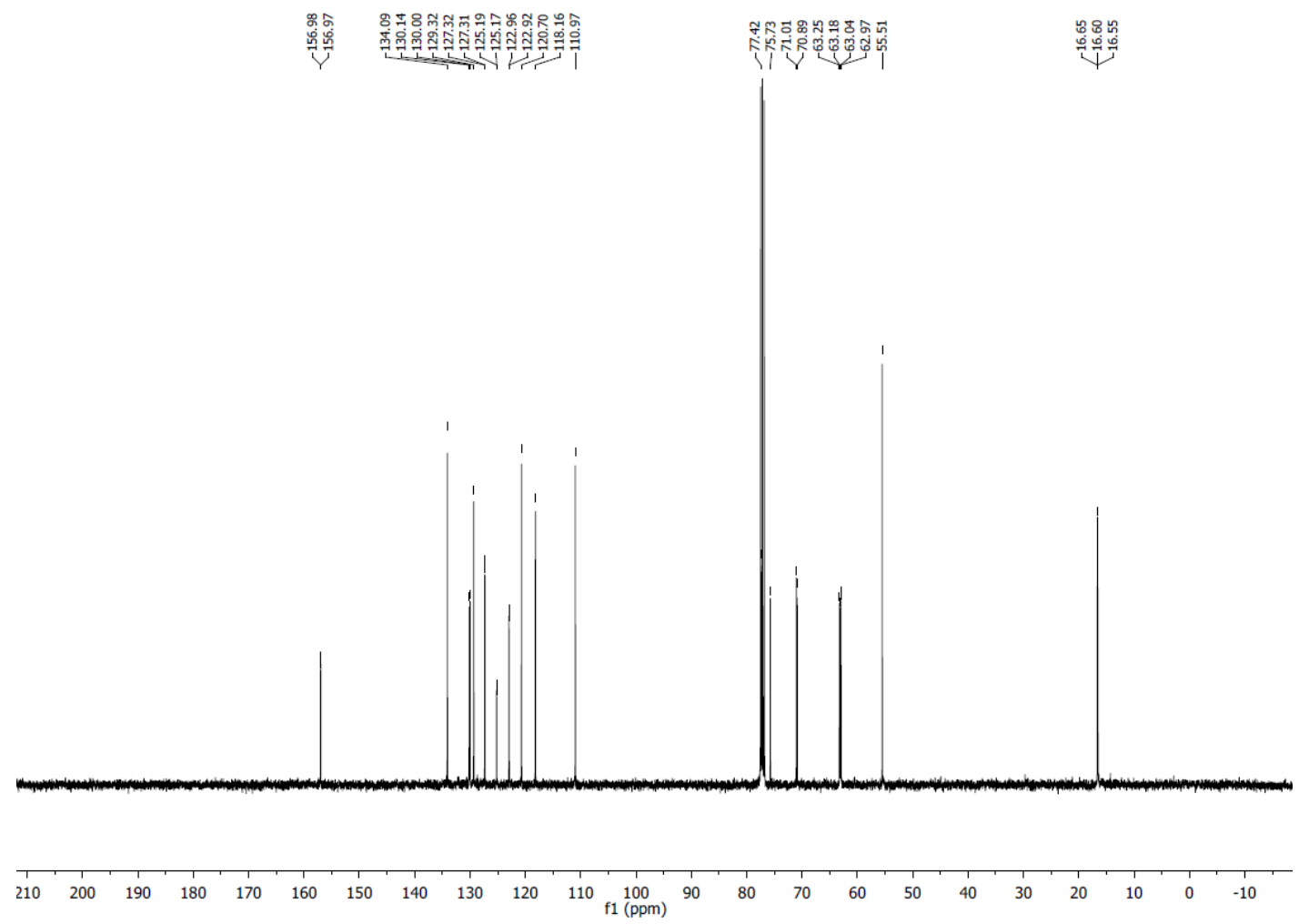


<smiles></smiles>

${ }^{1} \mathrm{H}-\mathrm{NMR}$ of compound $5 \mathrm{~s}\left(400 \mathrm{MHz}, \mathrm{CDCl}_{3}\right.$ )

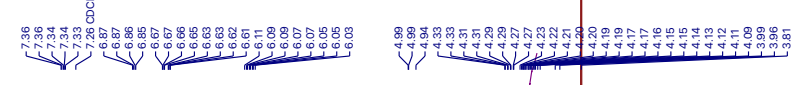
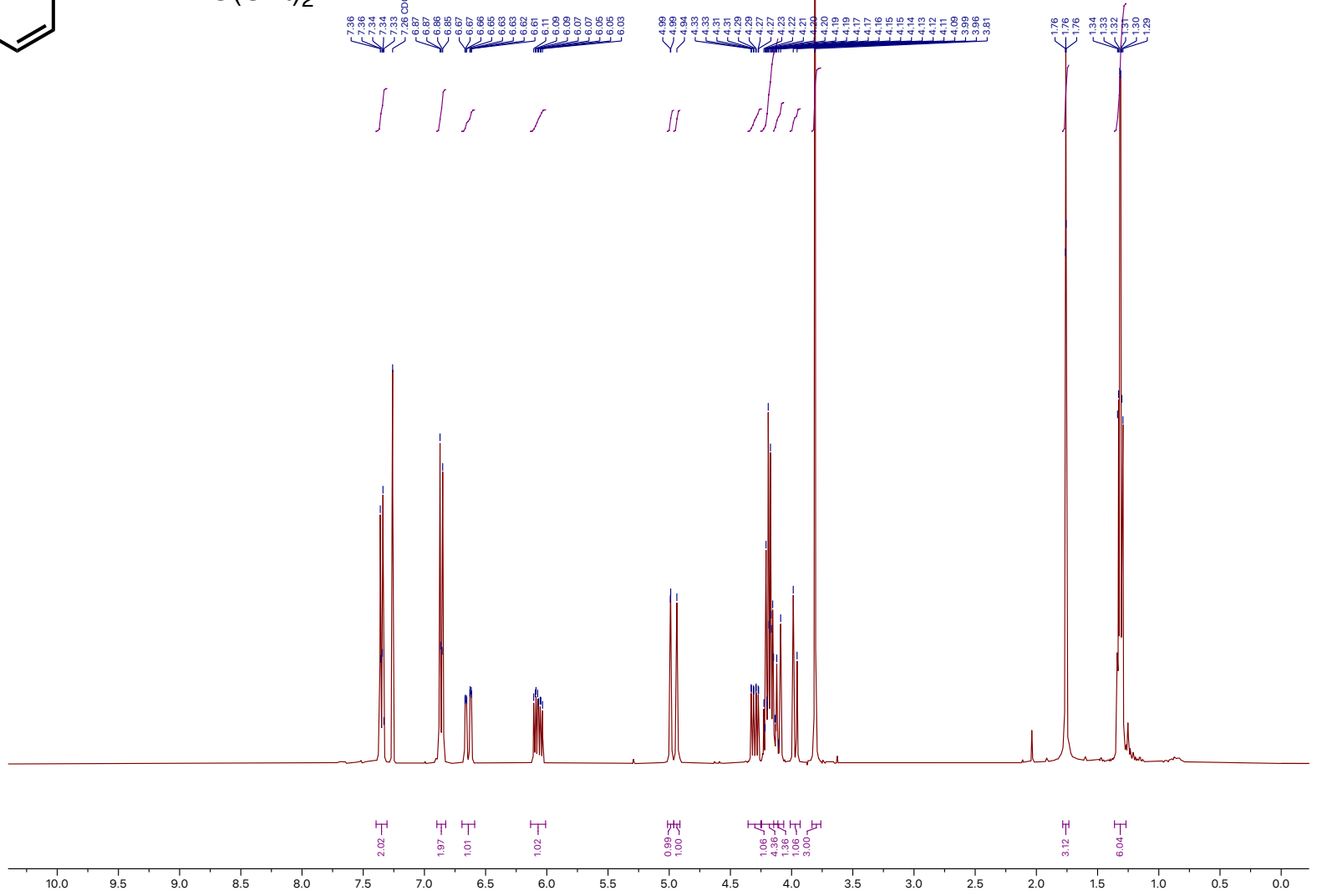

${ }^{13} \mathrm{C}$-NMR of compound 5s (101 MHz, $\left.\mathrm{CDCl}_{3}\right)$
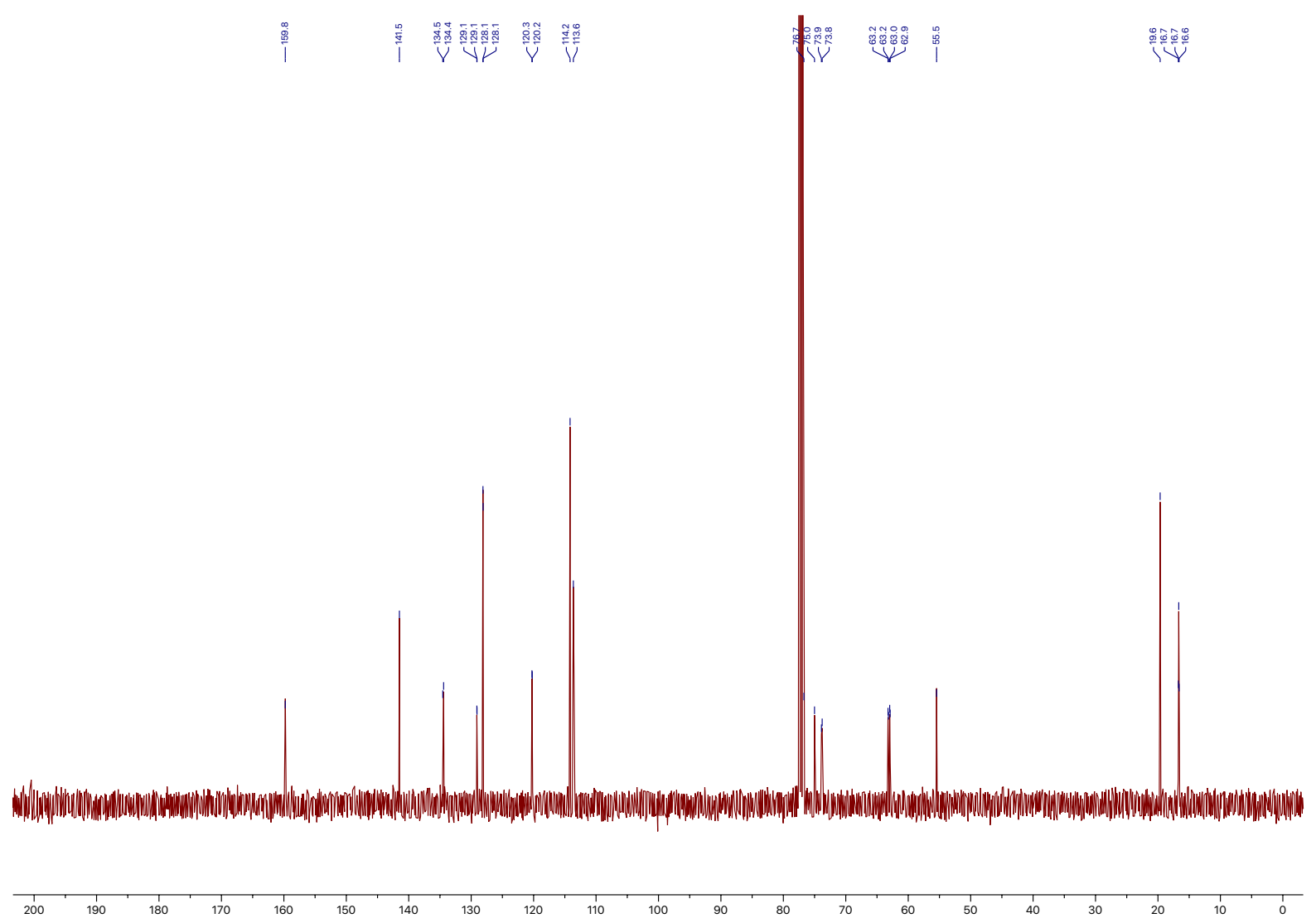


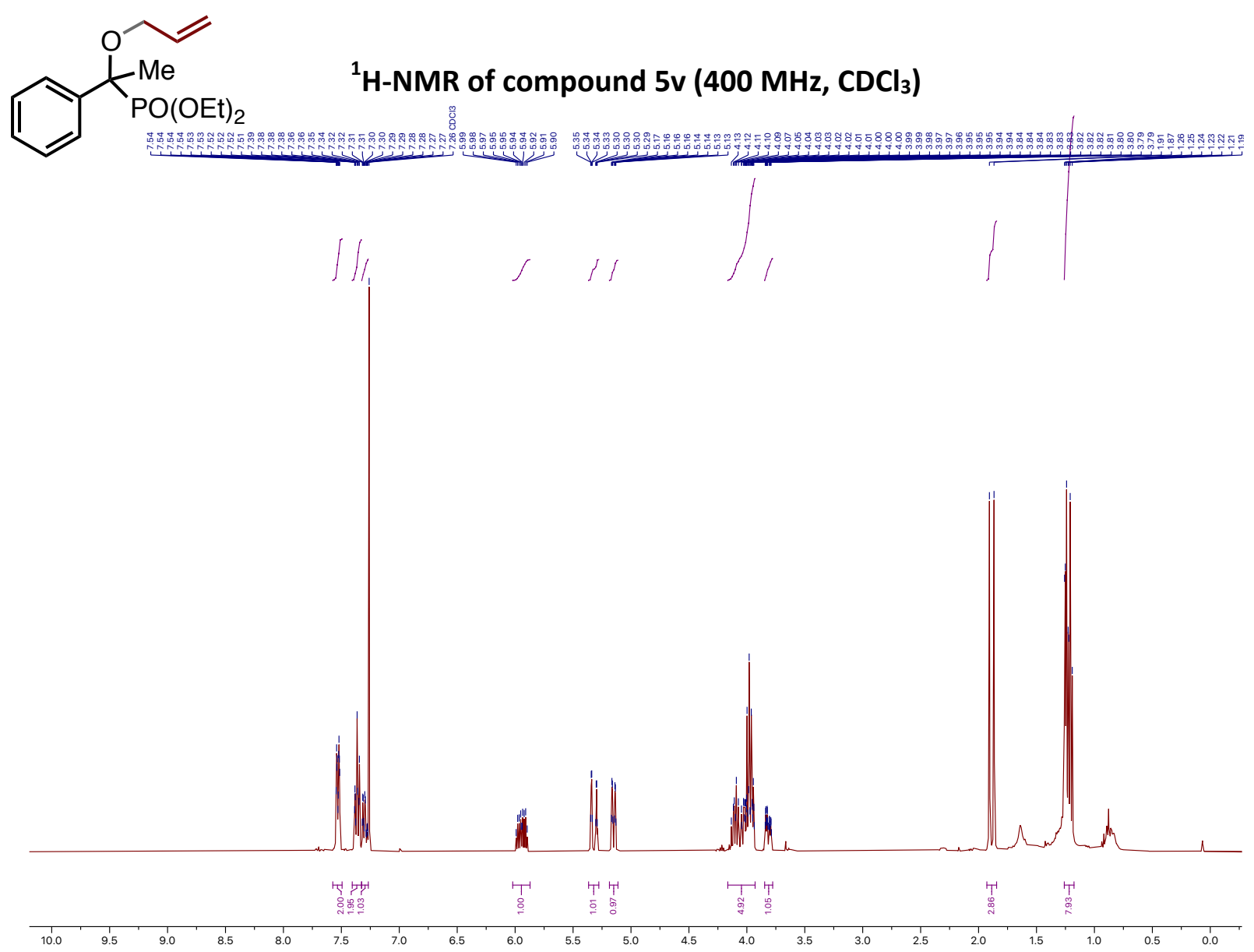

${ }^{13} \mathrm{C}-\mathrm{NMR}$ of compound $5 \mathrm{v}\left(101 \mathrm{MHz}, \mathrm{CDCl}_{3}\right)$

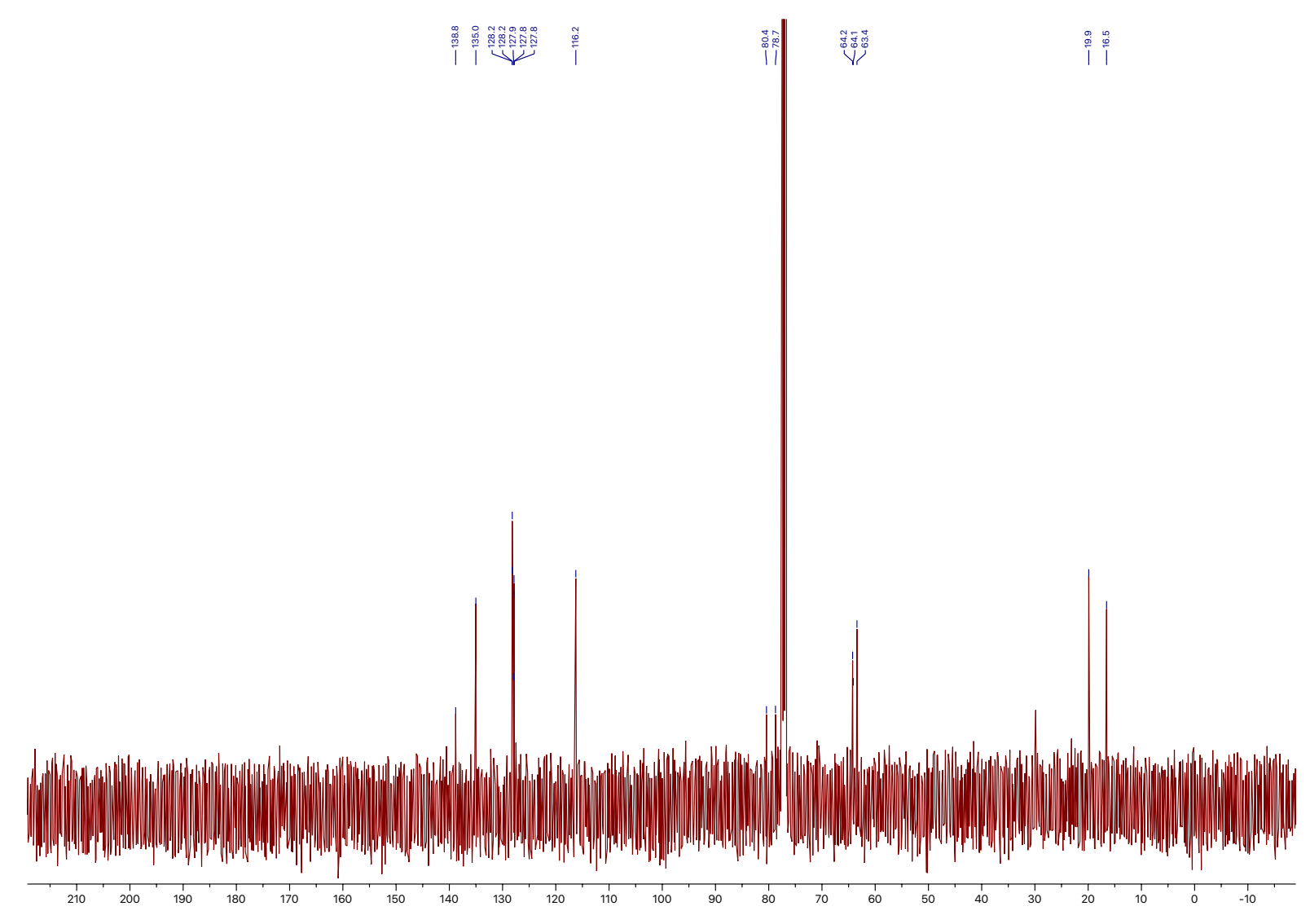


<smiles>C=CCOC(OCC)c1cnc2ccccc2c1</smiles>

${ }^{1} \mathrm{H}-\mathrm{NMR}$ of compound $5 \times\left(400 \mathrm{MHz}, \mathrm{CDCl}_{3}\right)$

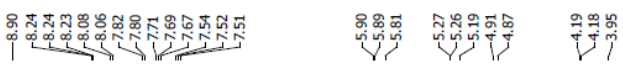

$\left.\iiint \int \|\right\}$

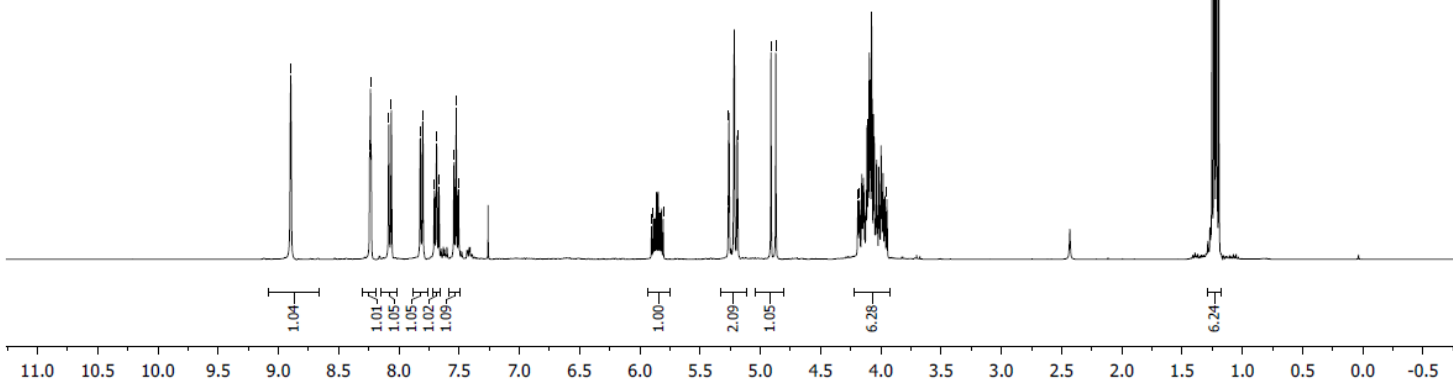

${ }^{13} \mathrm{C}$-NMR of compound $5 \times\left(101 \mathrm{MHz}, \mathrm{CDCl}_{3}\right)$

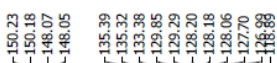

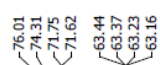

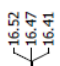

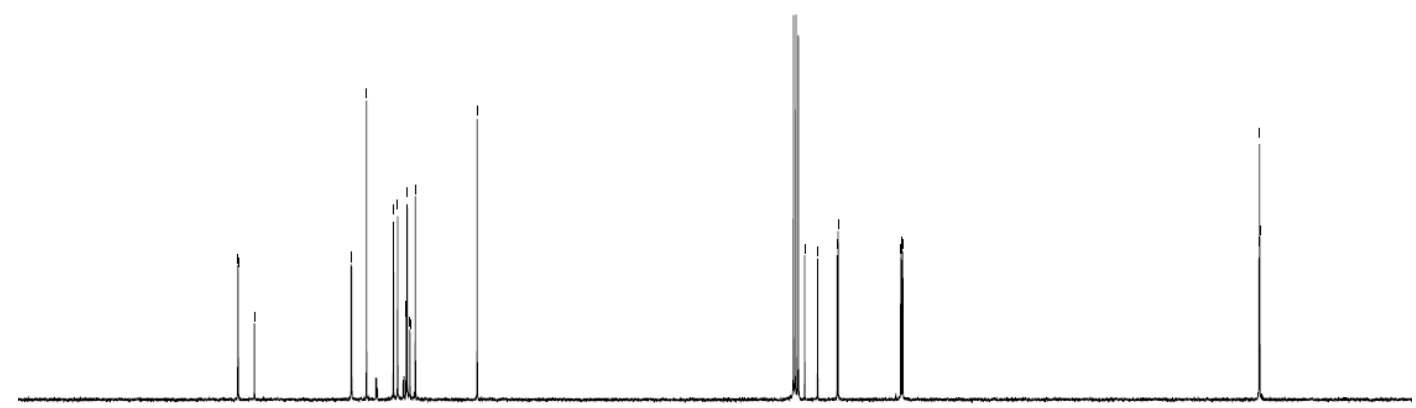

$\begin{array}{llll}170 & 160 & 150 & 140\end{array}$

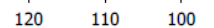

$60 \quad 50$

20 


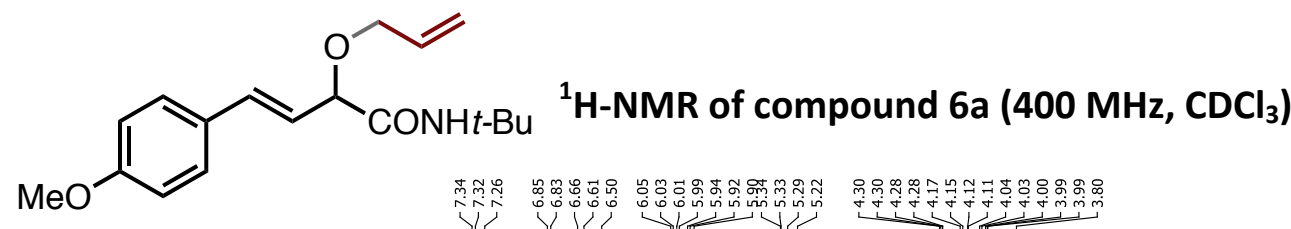

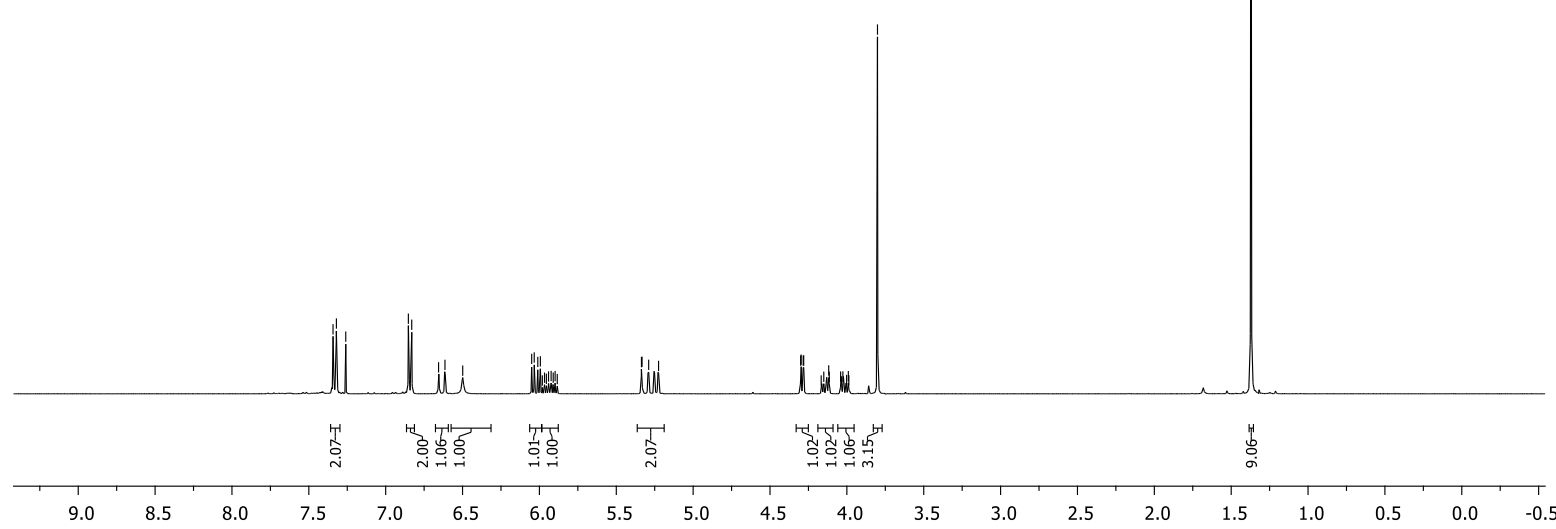

${ }^{13} \mathrm{C}-\mathrm{NMR}$ of compound $6 \mathrm{a}\left(101 \mathrm{MHz}, \mathrm{CDCl}_{3}\right)$

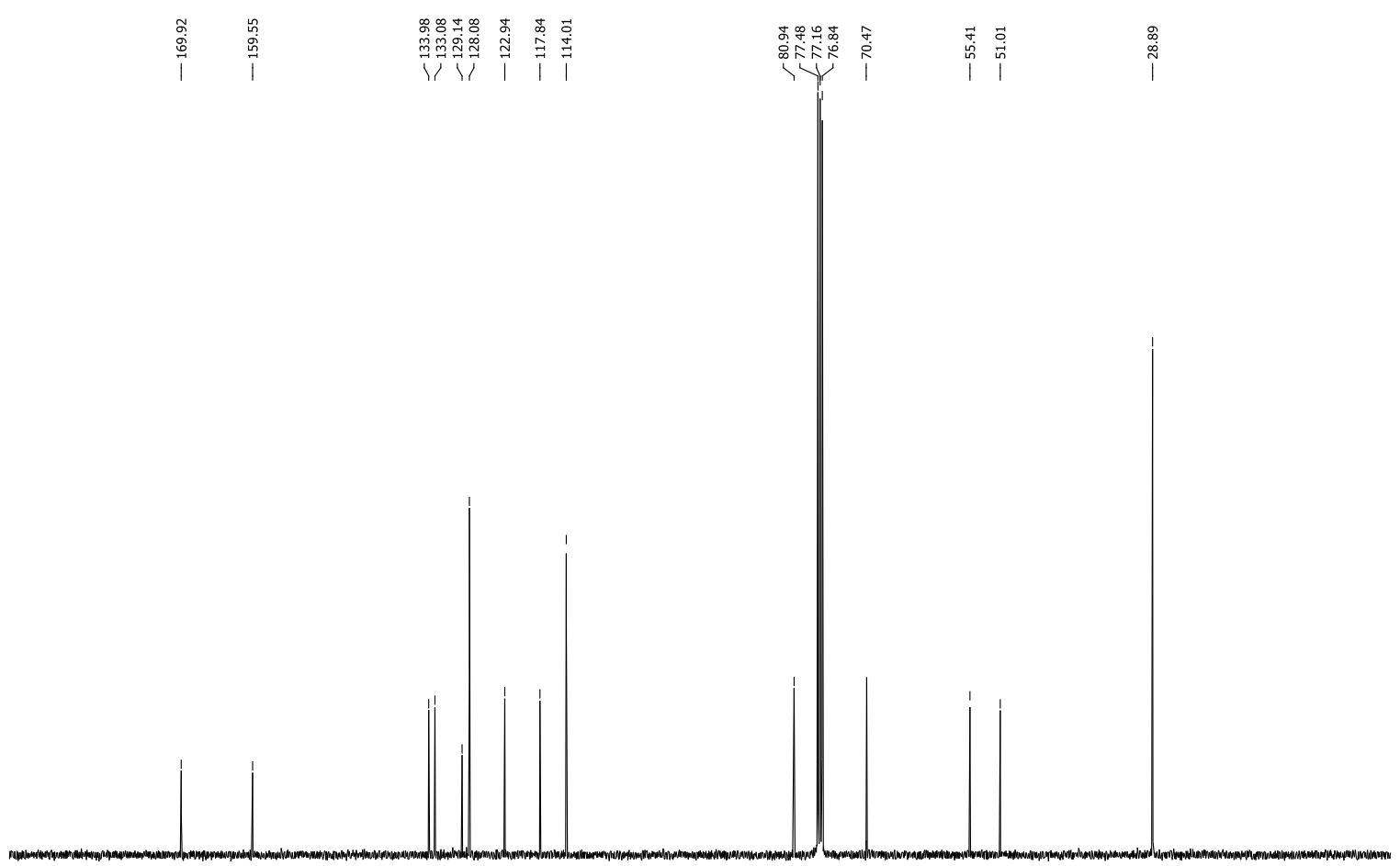




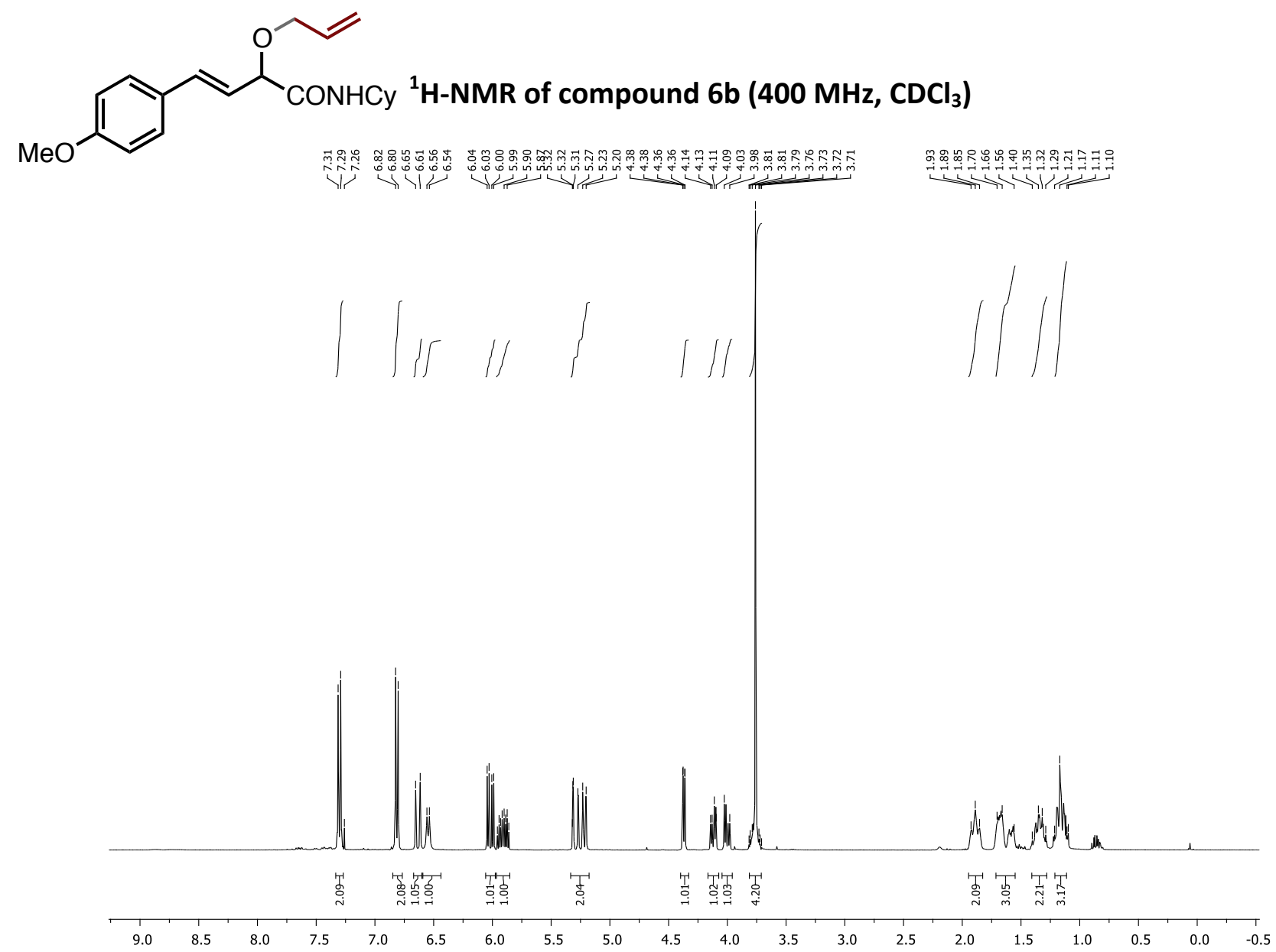

${ }^{13} \mathrm{C}-\mathrm{NMR}$ of compound $6 \mathrm{~b}\left(101 \mathrm{MHz}, \mathrm{CDCl}_{3}\right)$

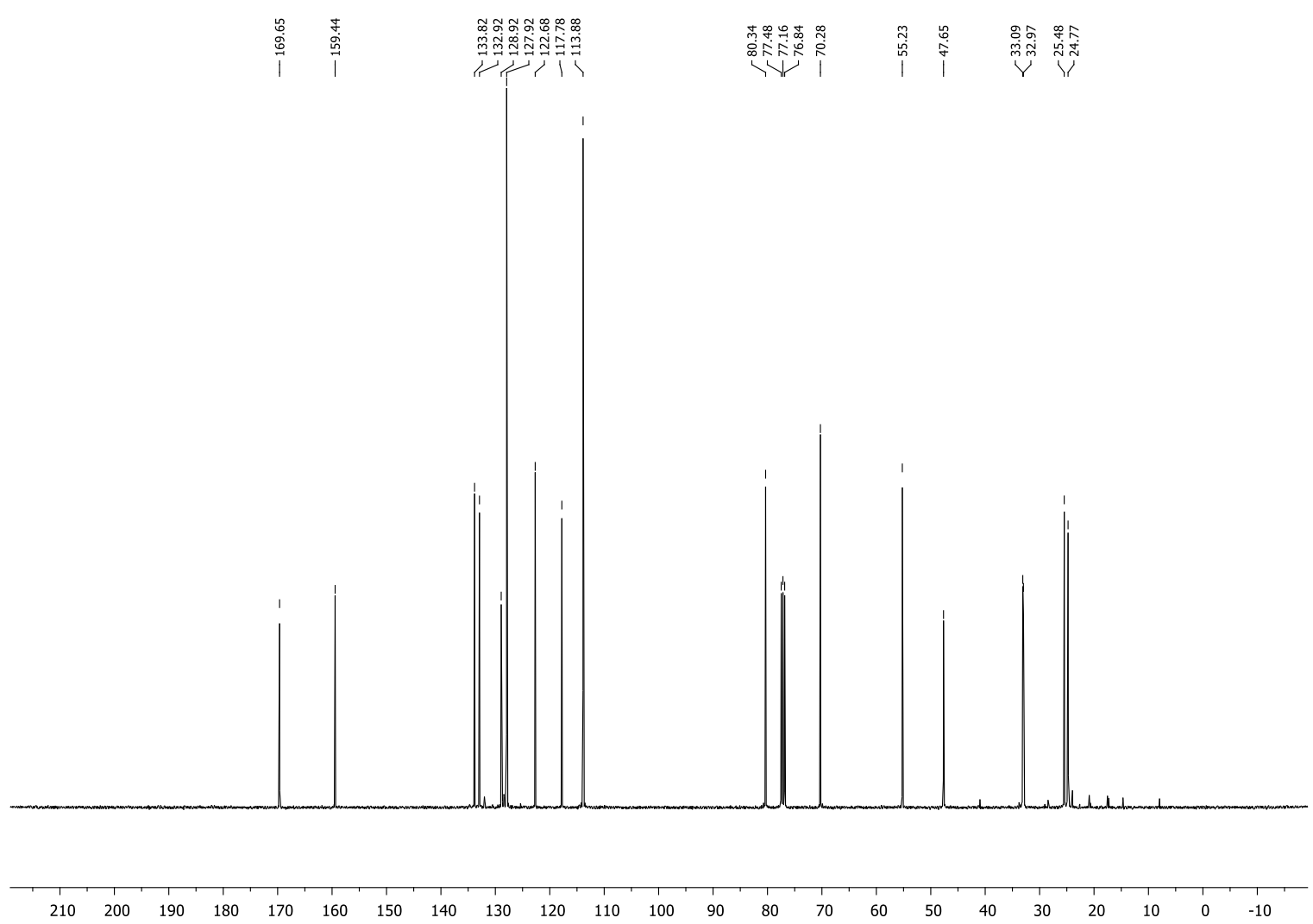




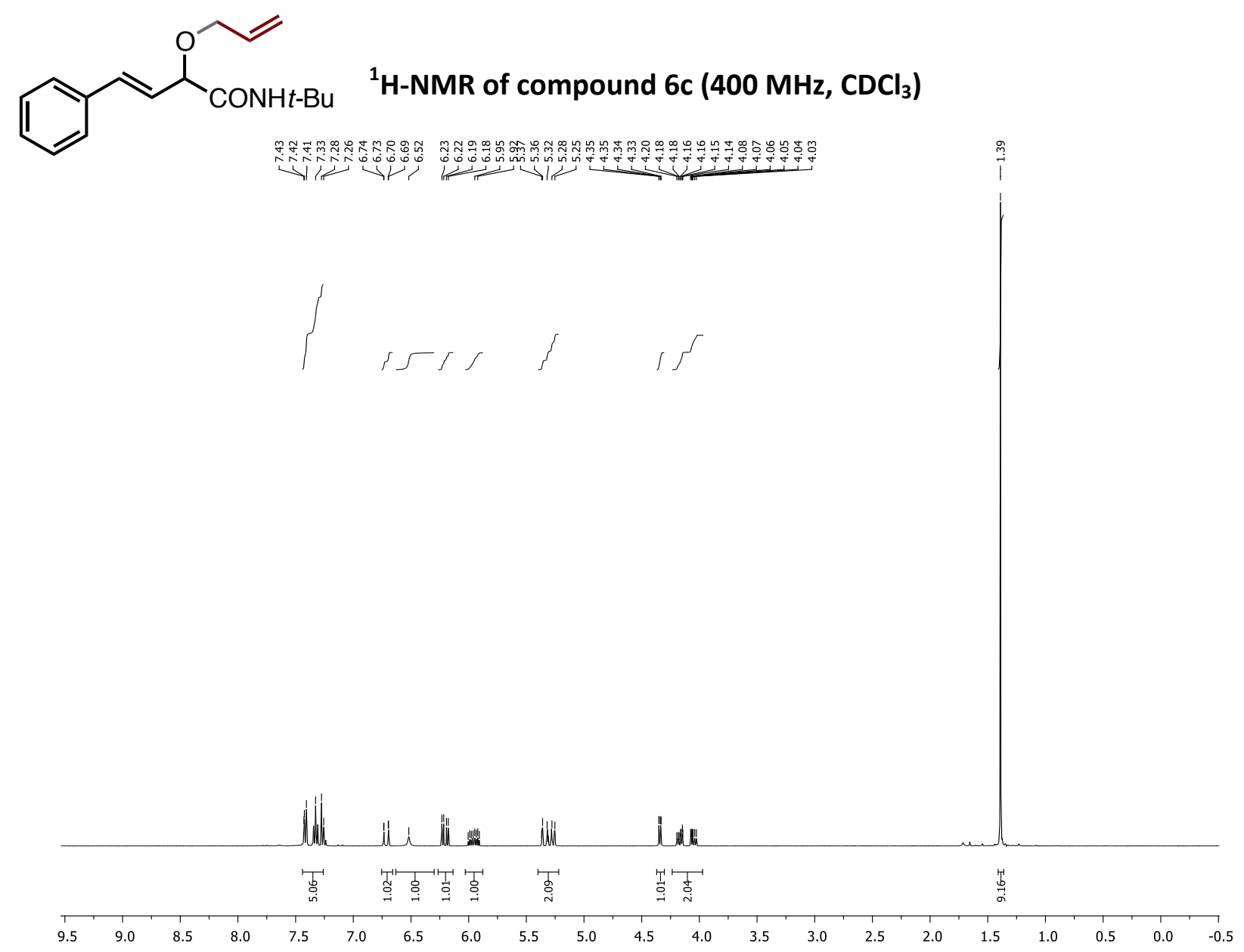

${ }^{13} \mathrm{C}-\mathrm{NMR}$ of compound $6 \mathrm{c}\left(101 \mathrm{MHz}, \mathrm{CDCl}_{3}\right)$

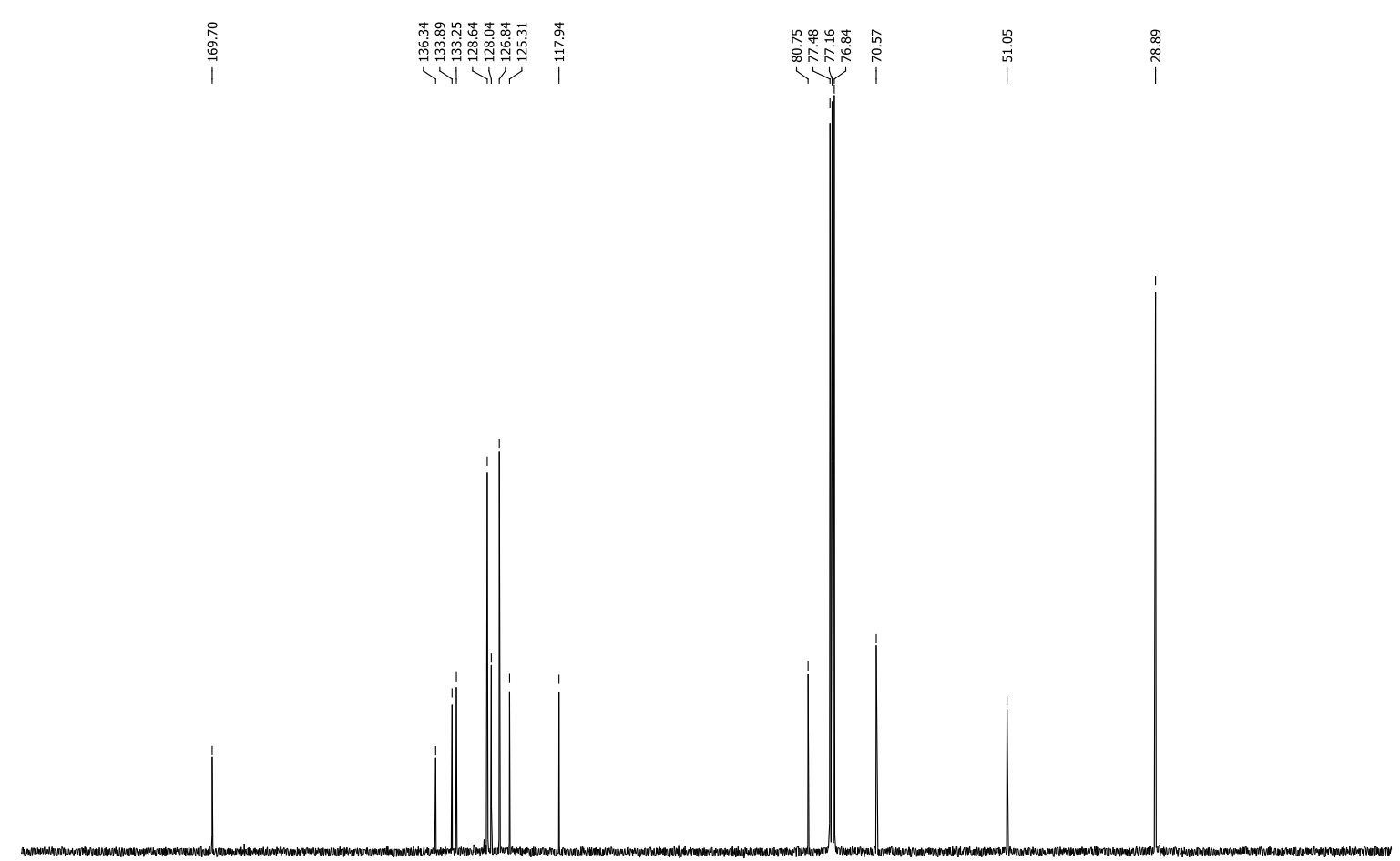

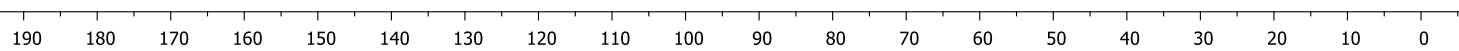


11 OONH

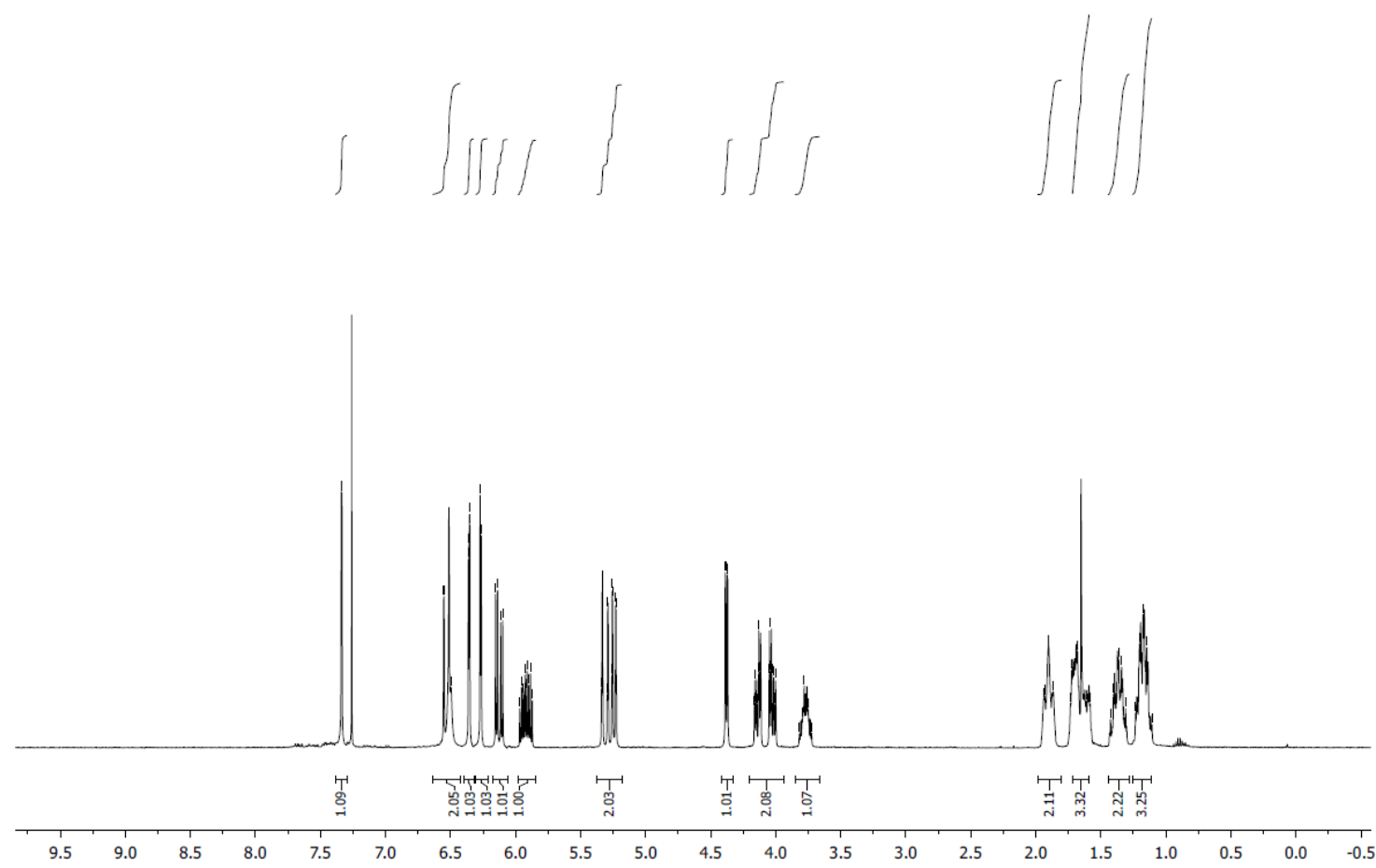

${ }^{13} \mathrm{C}-\mathrm{NMR}$ of compound $6 \mathrm{~d}\left(101 \mathrm{MHz}, \mathrm{CDCl}_{3}\right)$

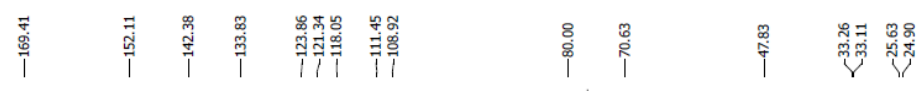

$\begin{array}{lllllllllllllllllllllll}210 & 200 & 190 & 180 & 170 & 160 & 150 & 140 & 130 & 120 & 110 & 100 & 90 & 80 & 70 & 60 & 50 & 40 & 30 & 20 & 10 & 0 & -10\end{array}$ 


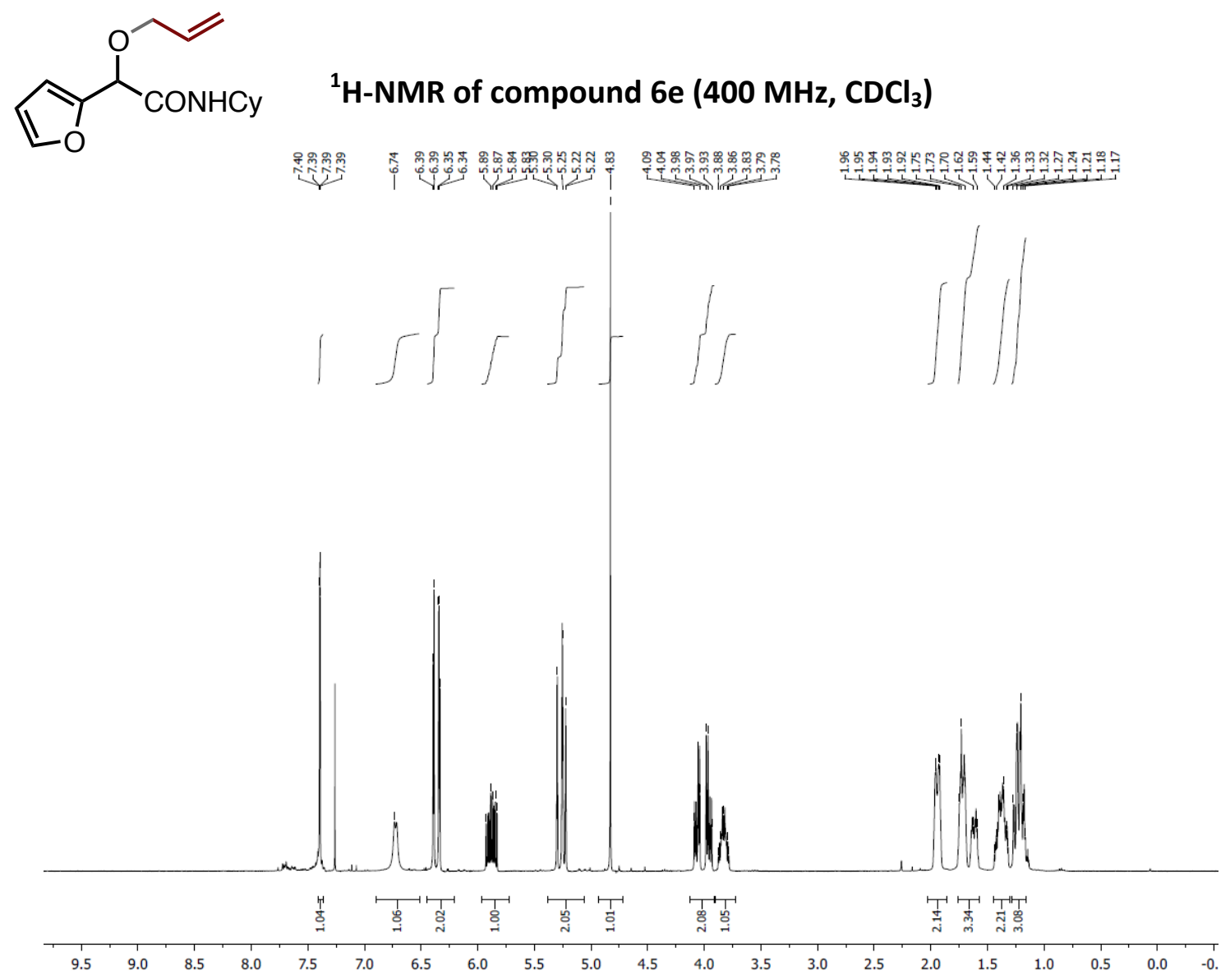

${ }^{13} \mathrm{C}-\mathrm{NMR}$ of compound $6 \mathrm{e}\left(101 \mathrm{MHz}, \mathrm{CDCl}_{3}\right)$

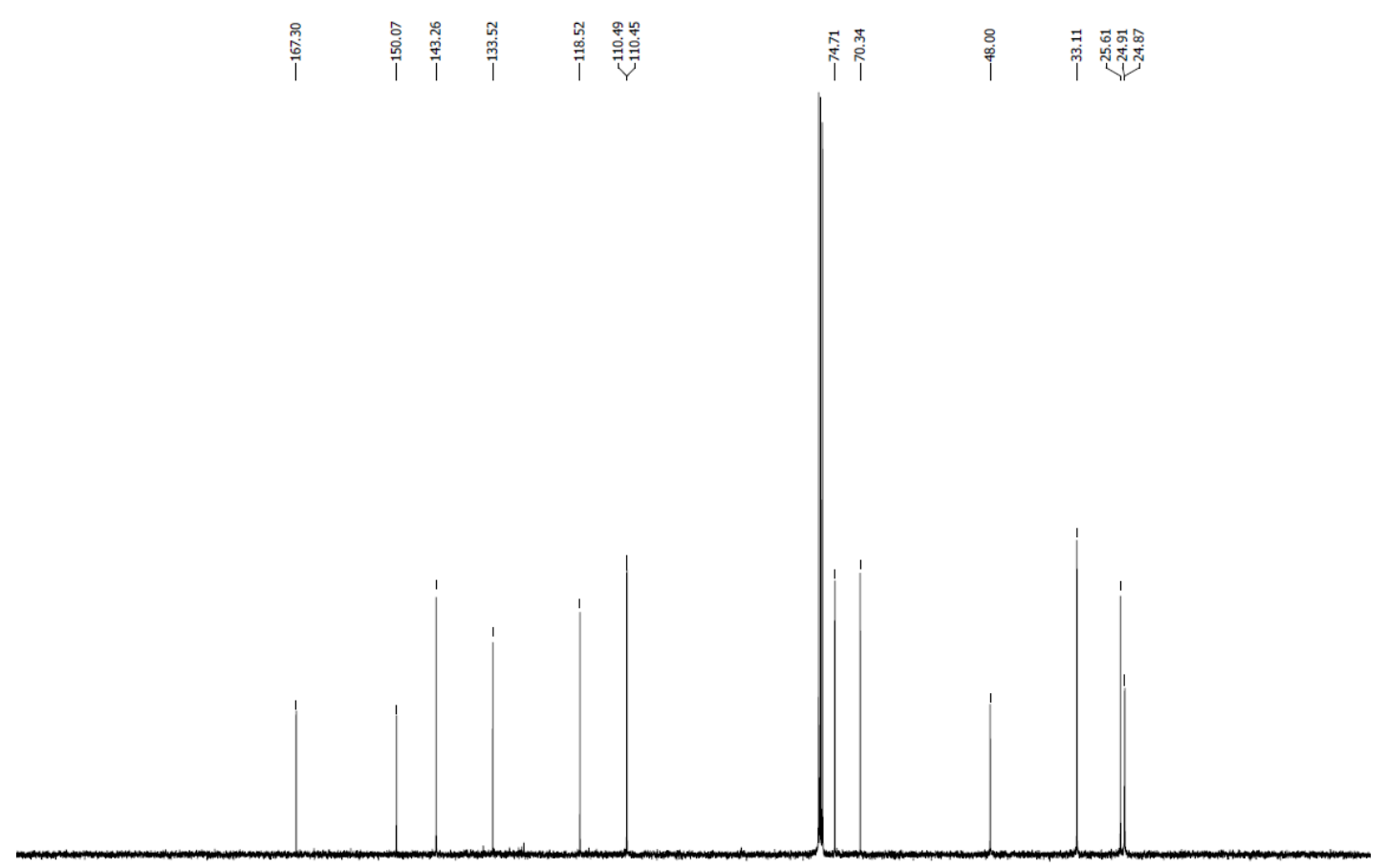

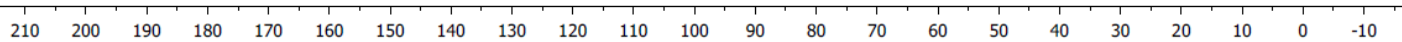


(1)

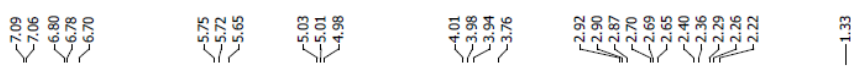

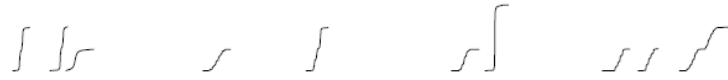

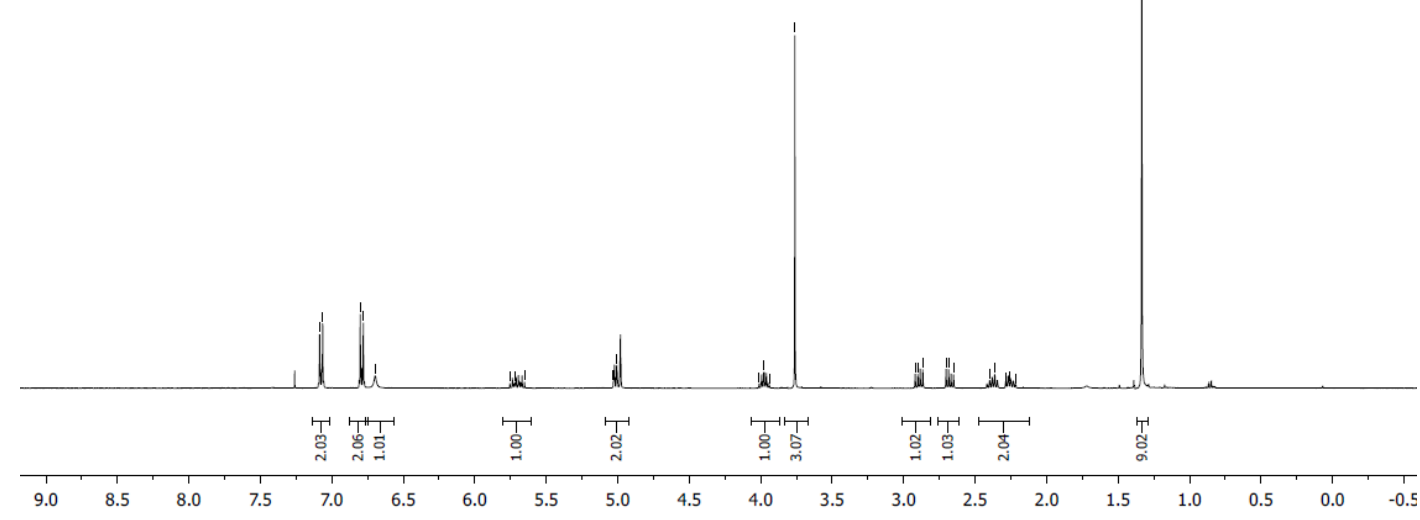

${ }^{13} \mathrm{C}-\mathrm{NMR}$ of compound $7 \mathrm{a}\left(101 \mathrm{MHz}, \mathrm{CDCl}_{3}\right)$

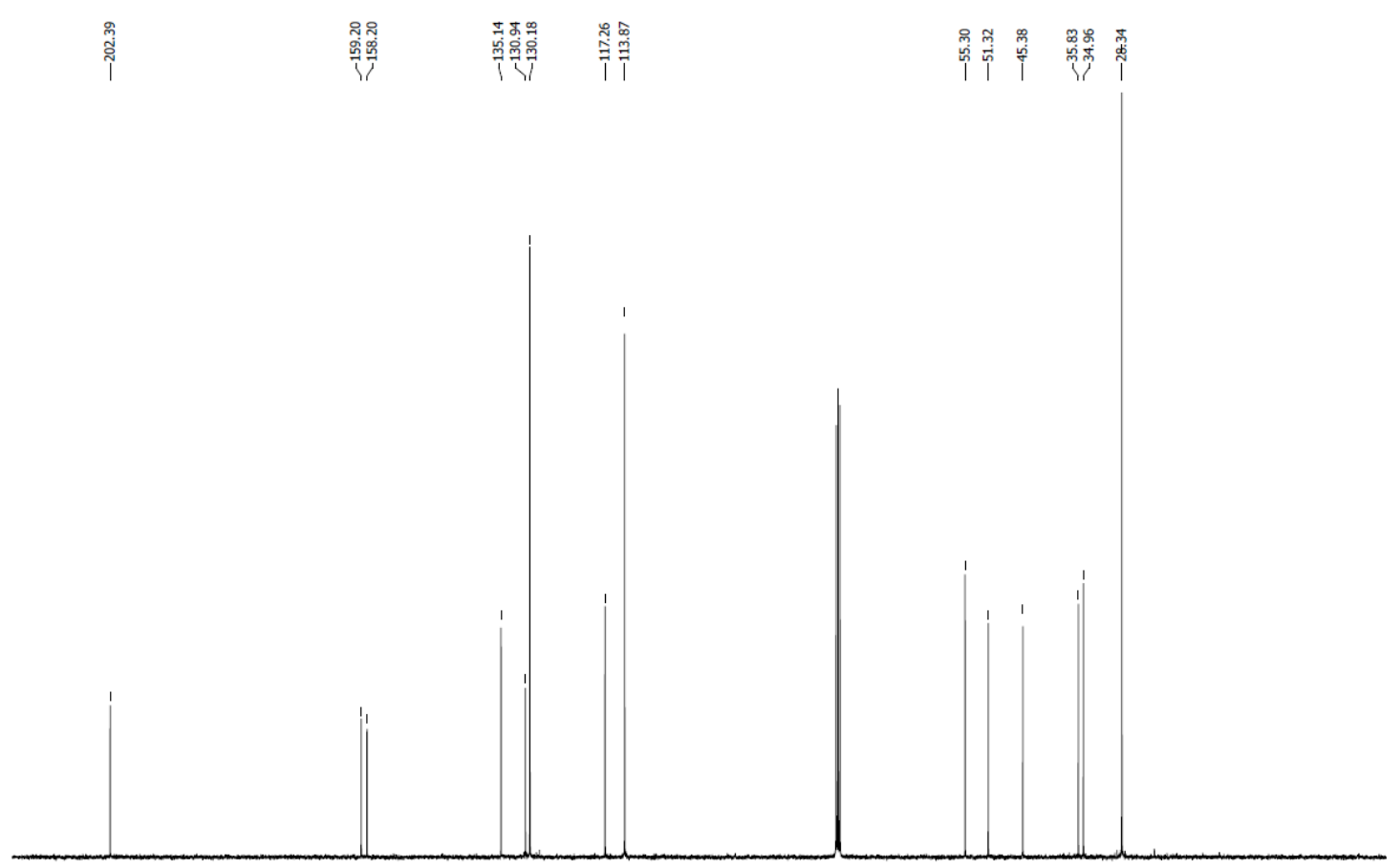

$\begin{array}{llllllllllllllllllllllllll}1 & 210 & 200 & 190 & 180 & 170 & 160 & 150 & 140 & 130 & 120 & 110 & 100 & 90 & 80 & 70 & 60 & 50 & 40 & 30 & 20 & 10 & 0 & -10\end{array}$ 
至

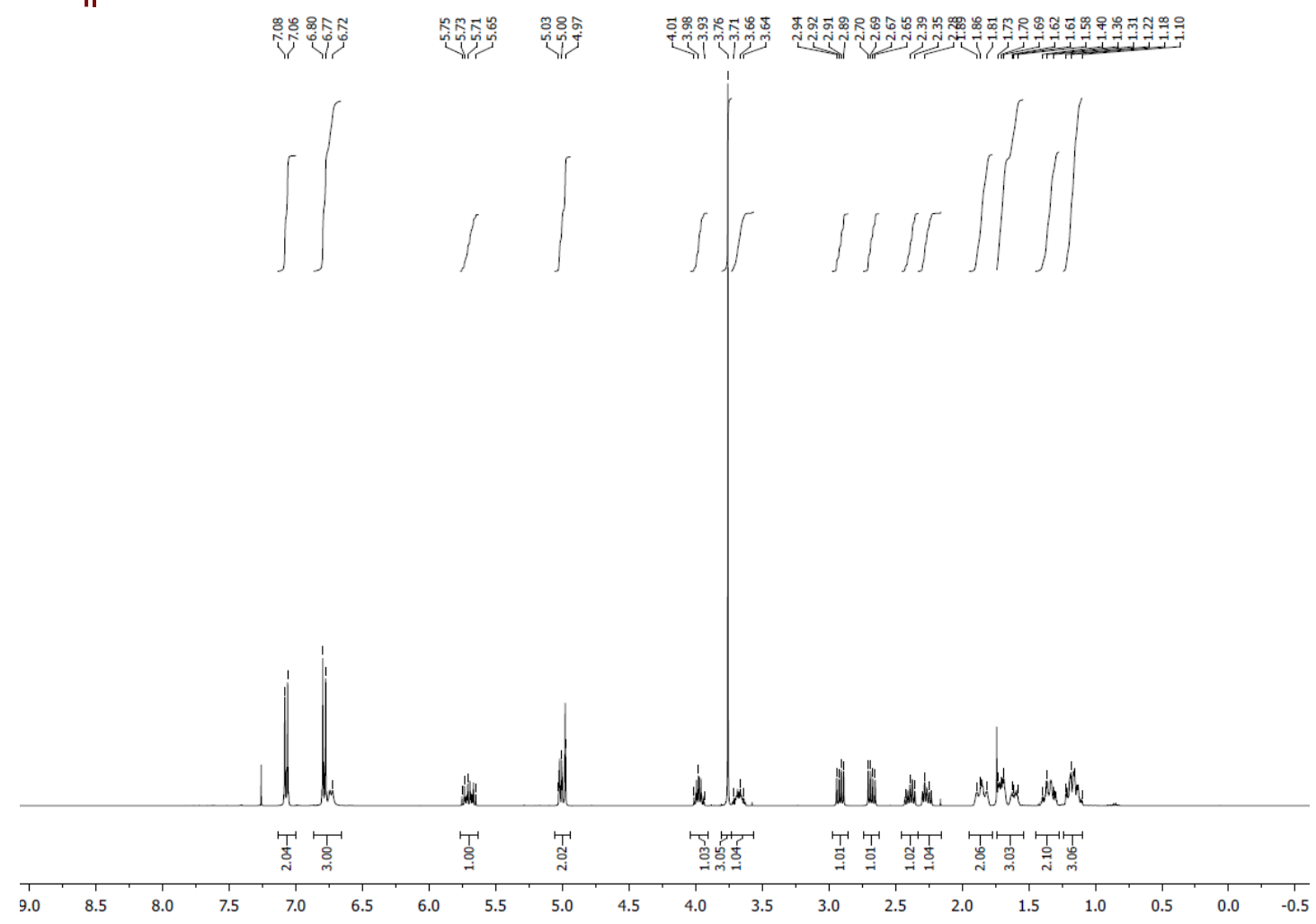

${ }^{13} \mathrm{C}-\mathrm{NMR}$ of compound 7b (101 MHz, $\mathrm{CDCl}_{3}$ )
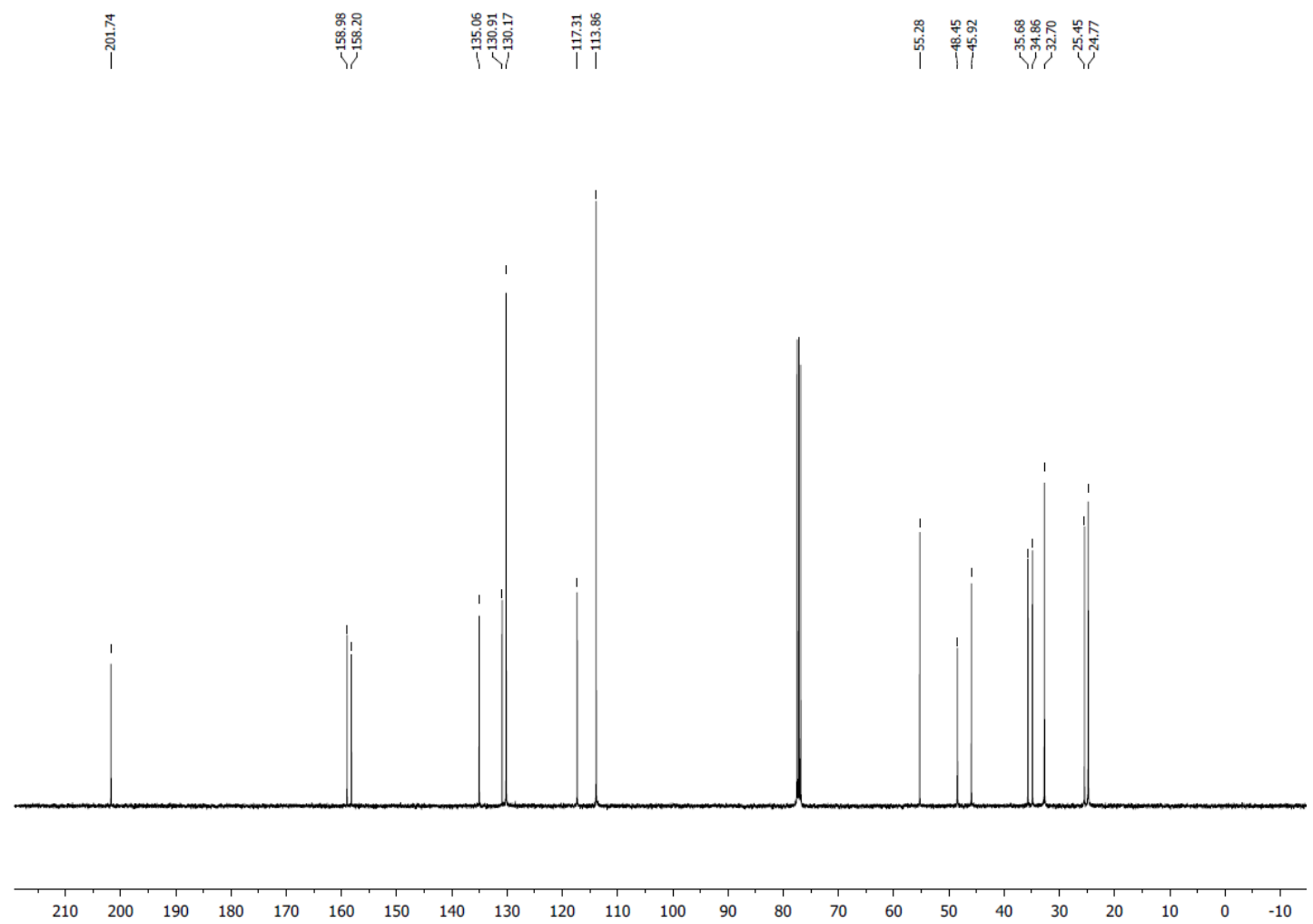
<smiles>C=CCC(Cc1ccccc1)C(=O)C(=O)NC(C)(C)C</smiles>

${ }^{1} \mathrm{H}-\mathrm{NMR}$ of compound Tc (400 $\left.\mathrm{MHz}, \mathrm{CDCl}_{3}\right)$

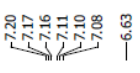

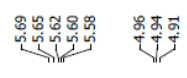

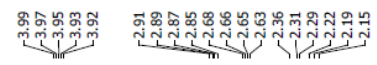

$\stackrel{8}{1}$

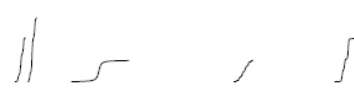

d t J

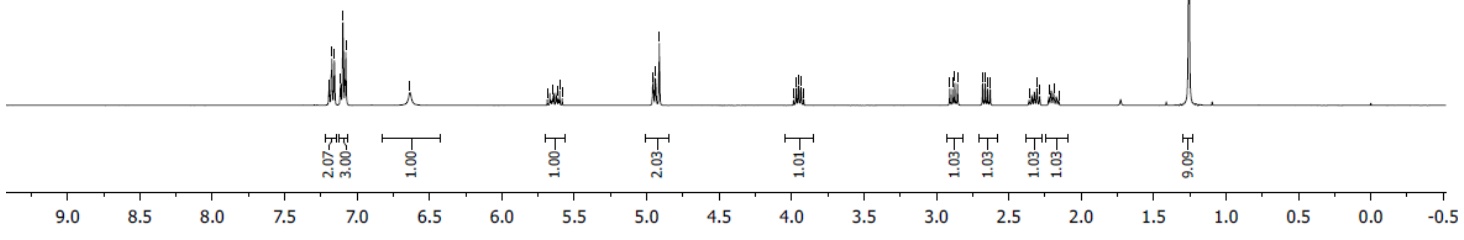

${ }^{13} \mathrm{C}$-NMR of compound Tc (101 MHz, $\left.\mathrm{CDCl}_{3}\right)$
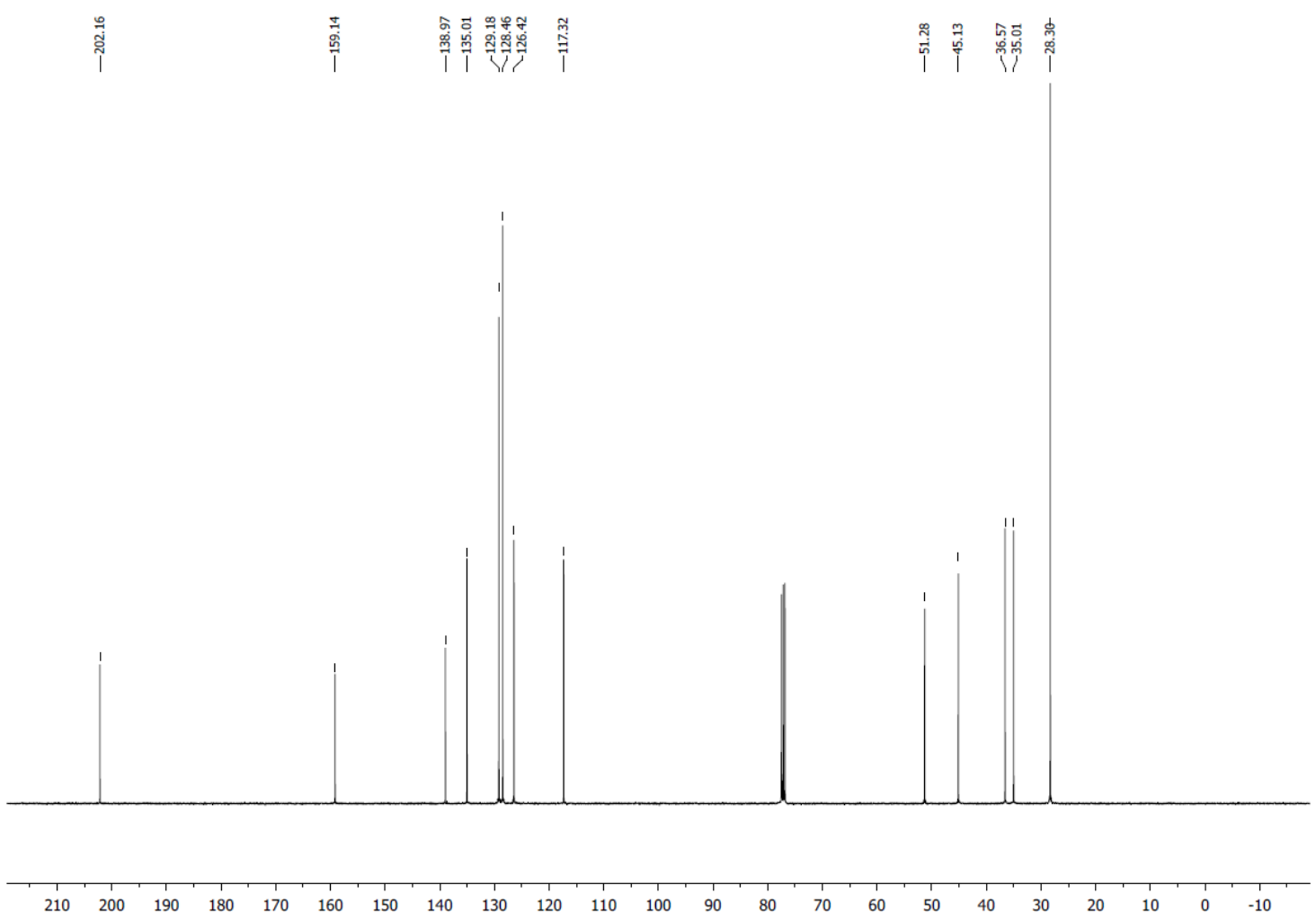

S28 
${ }^{1} \mathrm{H}-\mathrm{NMR}$ of compound $7 \mathrm{~d}\left(400 \mathrm{MHz}, \mathrm{CDCl}_{3}\right)$

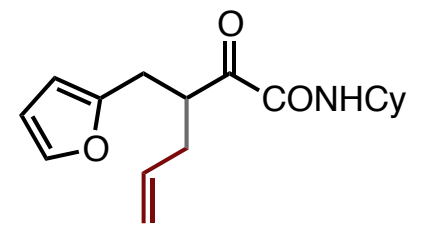

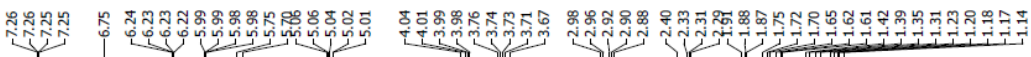
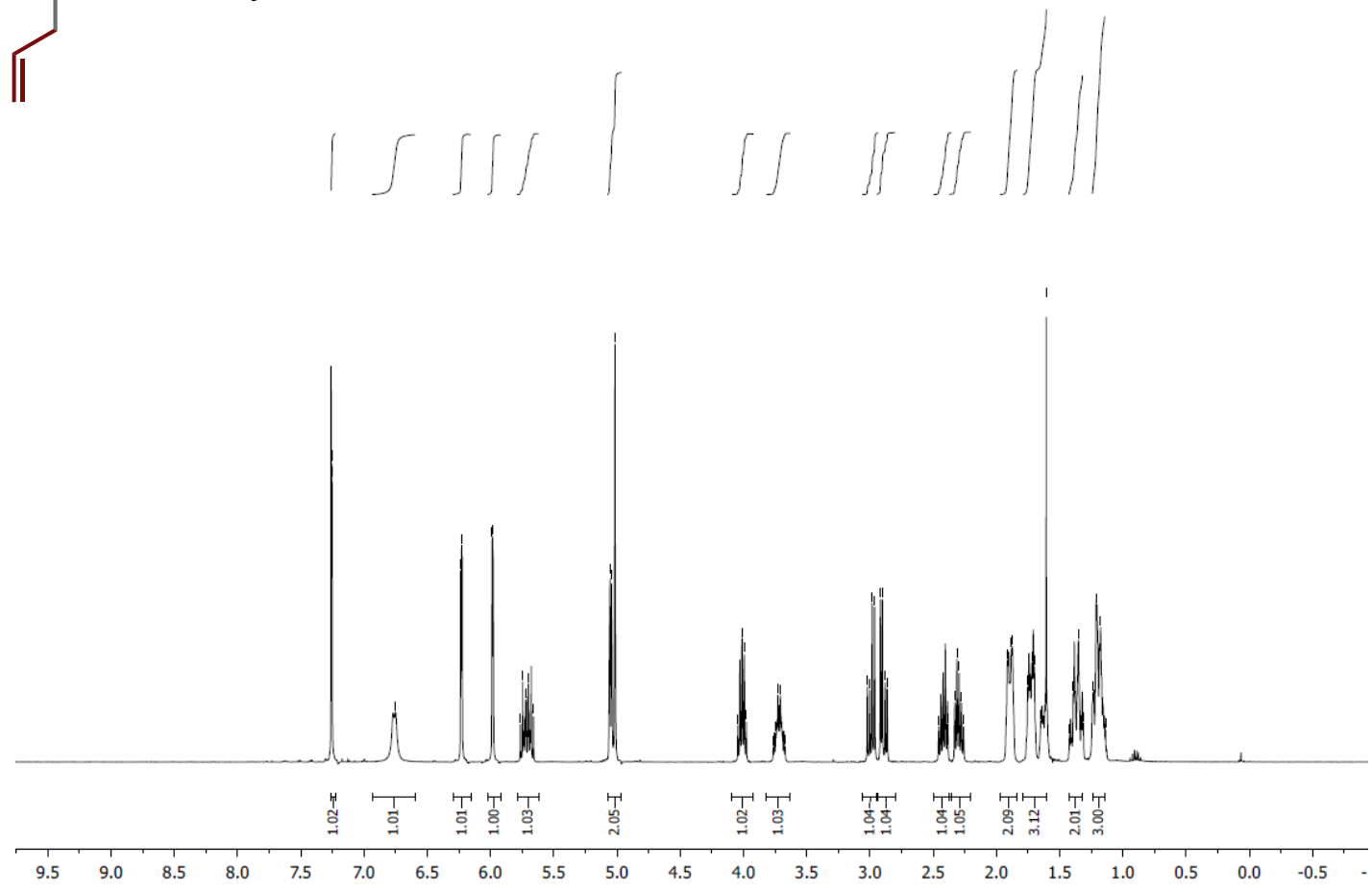

${ }^{13} \mathrm{C}$-NMR of compound 7d (101 MHz, $\left.\mathrm{CDCl}_{3}\right)$

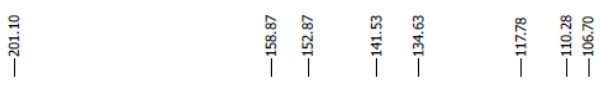

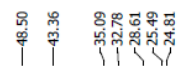

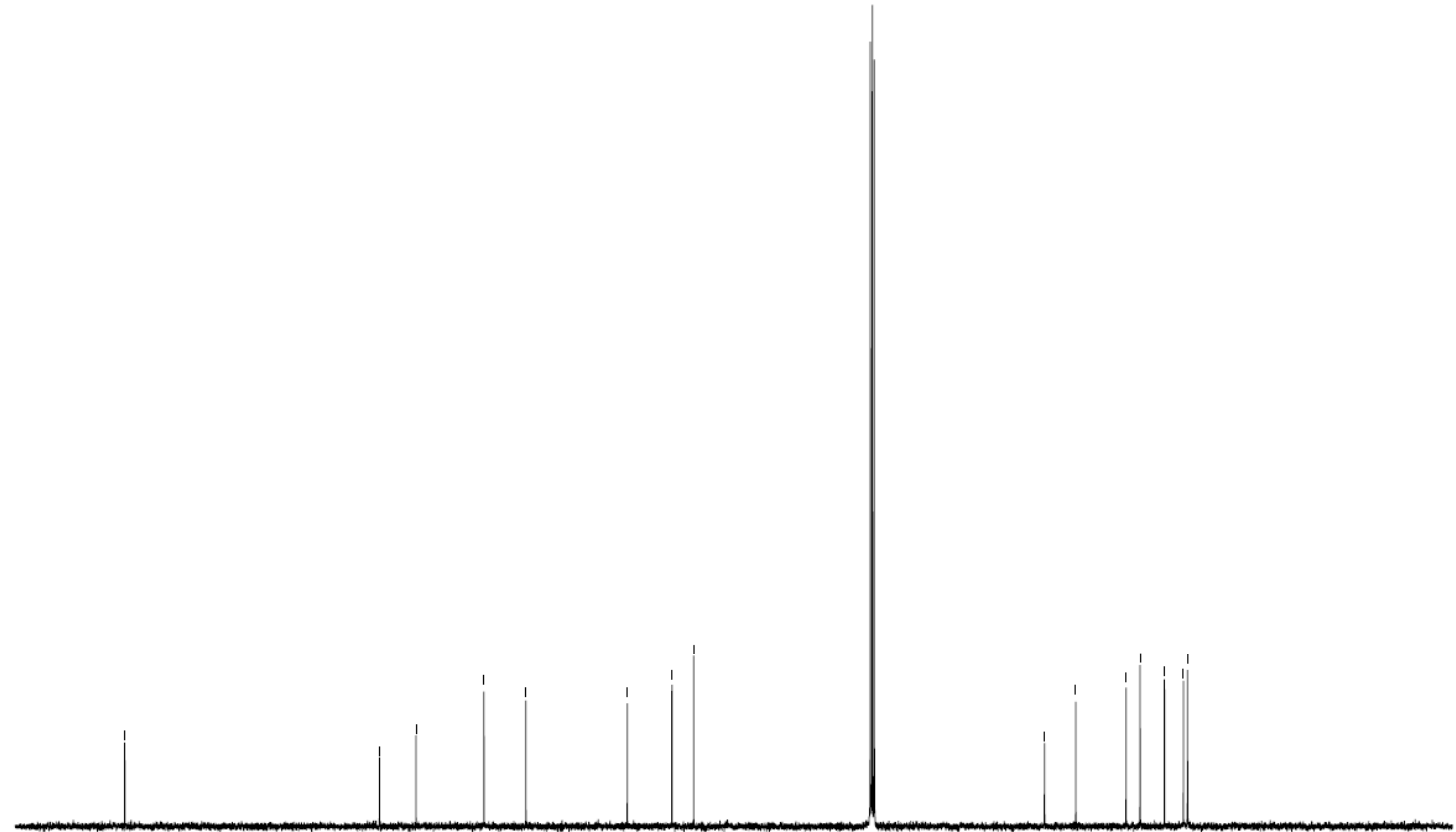


${ }^{1} \mathrm{H}-\mathrm{NMR}$ of compound 9a $\left(400 \mathrm{MHz}, \mathrm{CDCl}_{3}\right)$

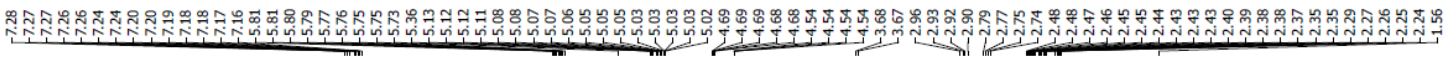<smiles>C=CCC(Cc1ccccc1)C(=O)NCC(=C)C</smiles>
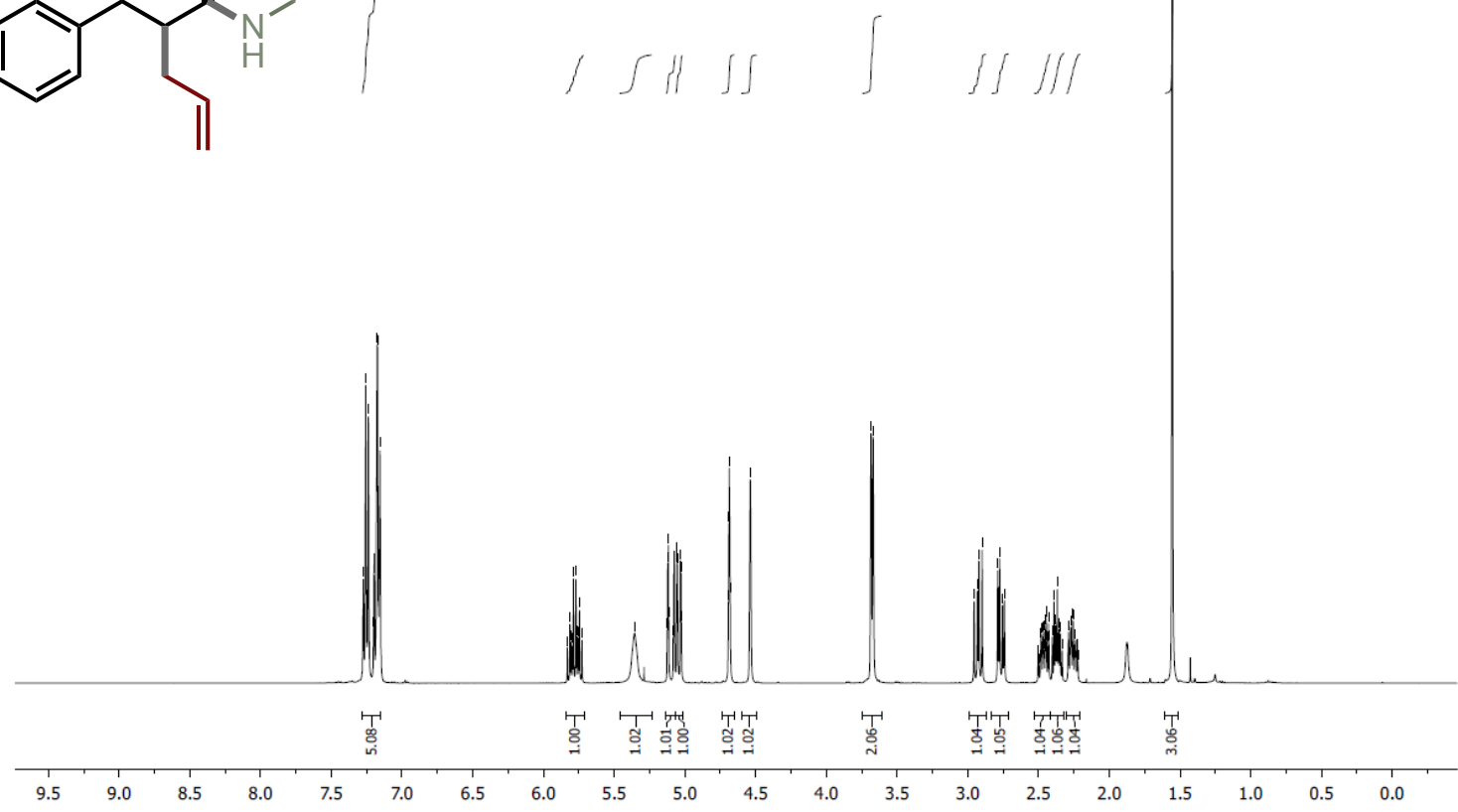

${ }^{13} \mathrm{C}-\mathrm{NMR}$ of compound 9a (101 $\left.\mathrm{MHz}, \mathrm{CDCl}_{3}\right)$
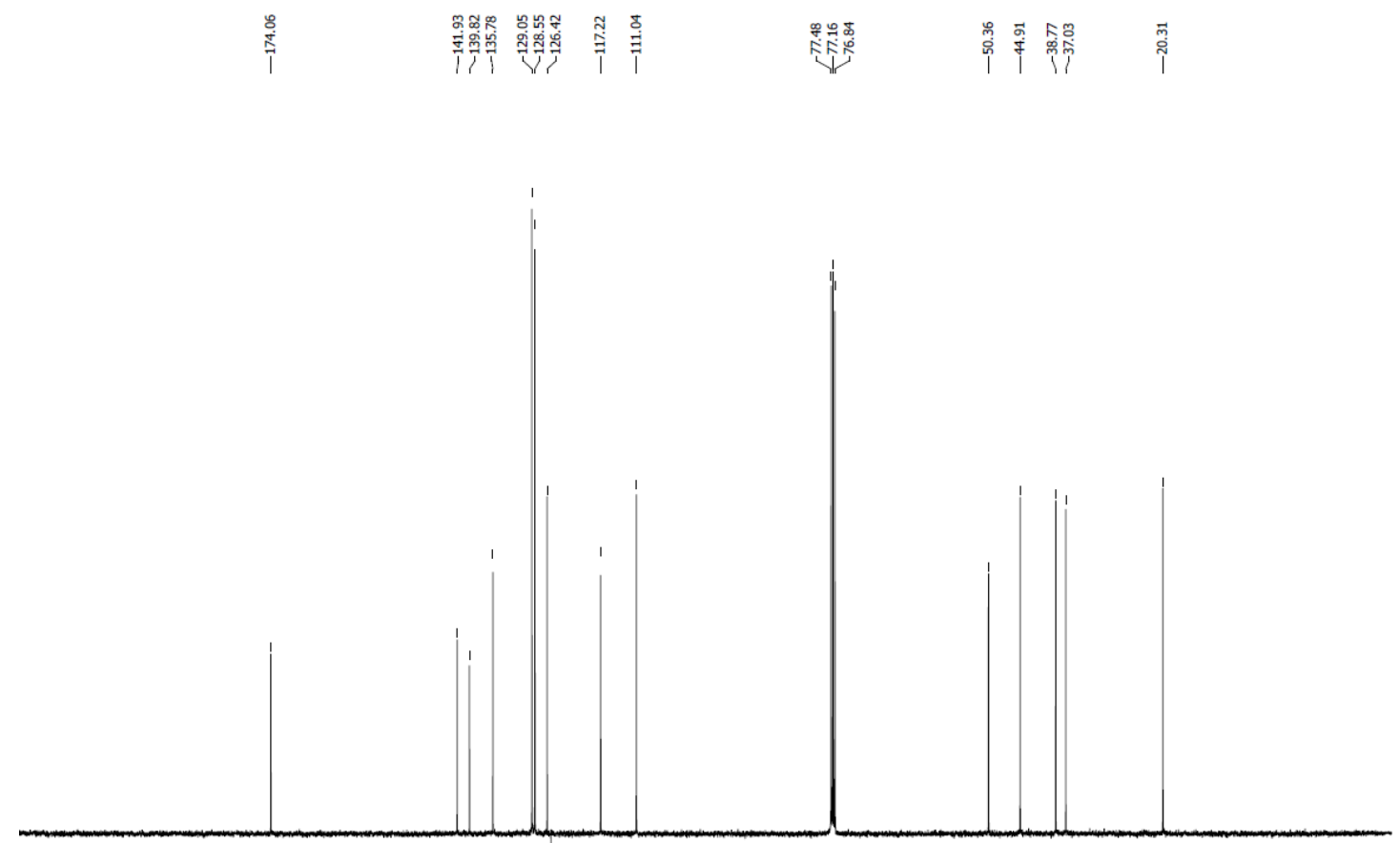

$\begin{array}{llllllllllllllllllllllllll}210 & 200 & 190 & 180 & 170 & 160 & 150 & 140 & 130 & 120 & 110 & 100 & 90 & 80 & 70 & 60 & 50 & 40 & 30 & 20 & 10 & 0 & -10\end{array}$ 


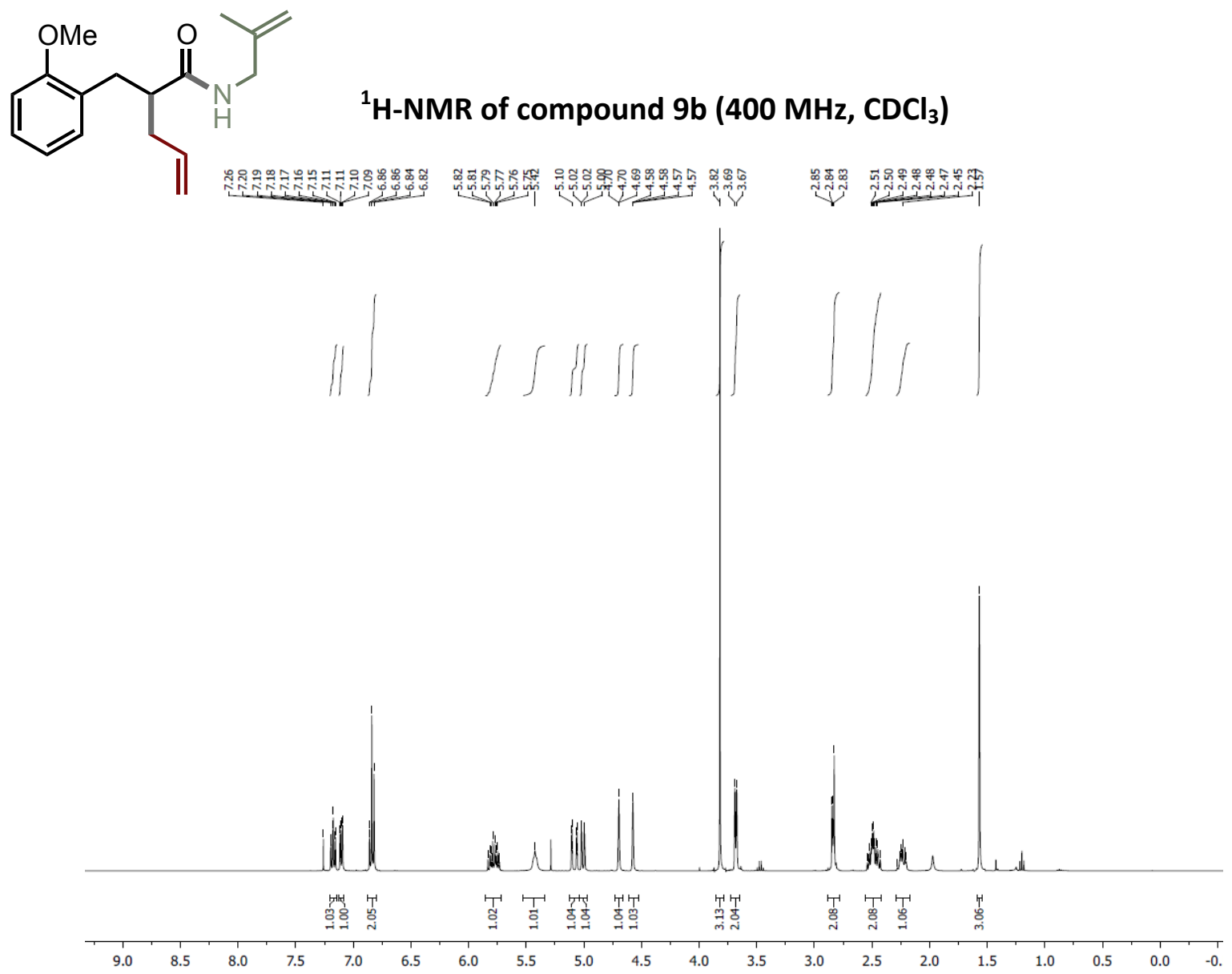

${ }^{13} \mathrm{C}-\mathrm{NMR}$ of compound $9 \mathrm{~b}\left(101 \mathrm{MHz}, \mathrm{CDCl}_{3}\right)$

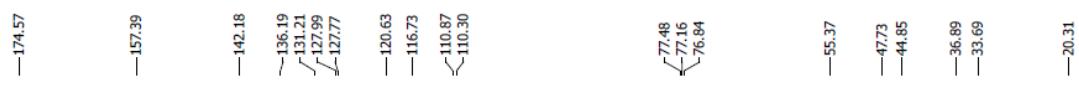

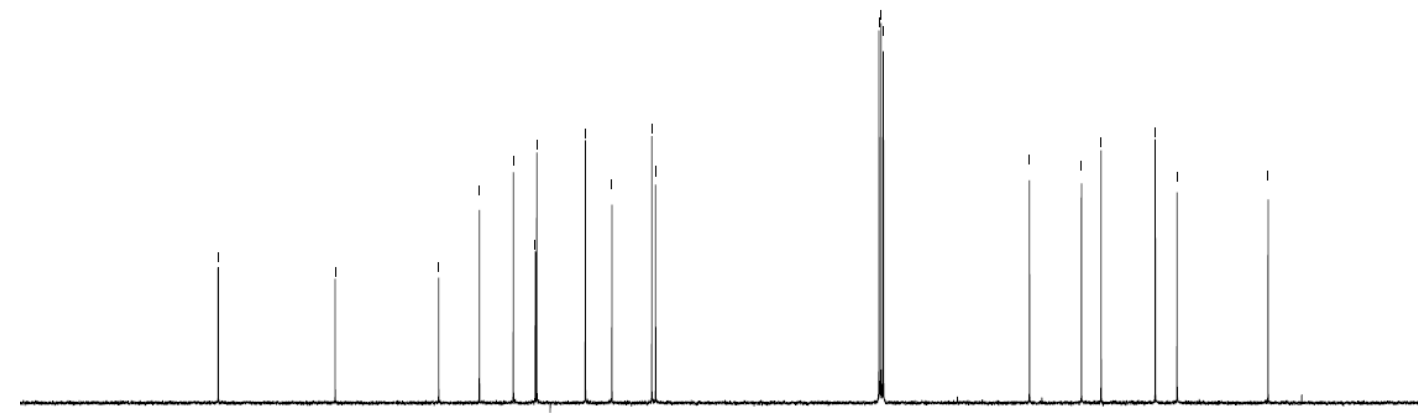

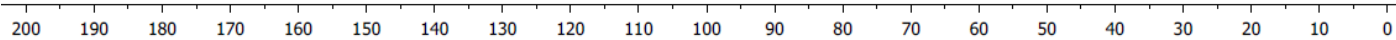


<smiles>C=CCC(Cc1ccc(OC)cc1)C(=O)NCC(=C)C</smiles>

${ }^{1} \mathrm{H}-\mathrm{NMR}$ of compound $9 \mathrm{c}\left(400 \mathrm{MHz}, \mathrm{CDCl}_{3}\right)$

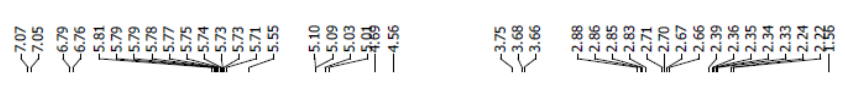

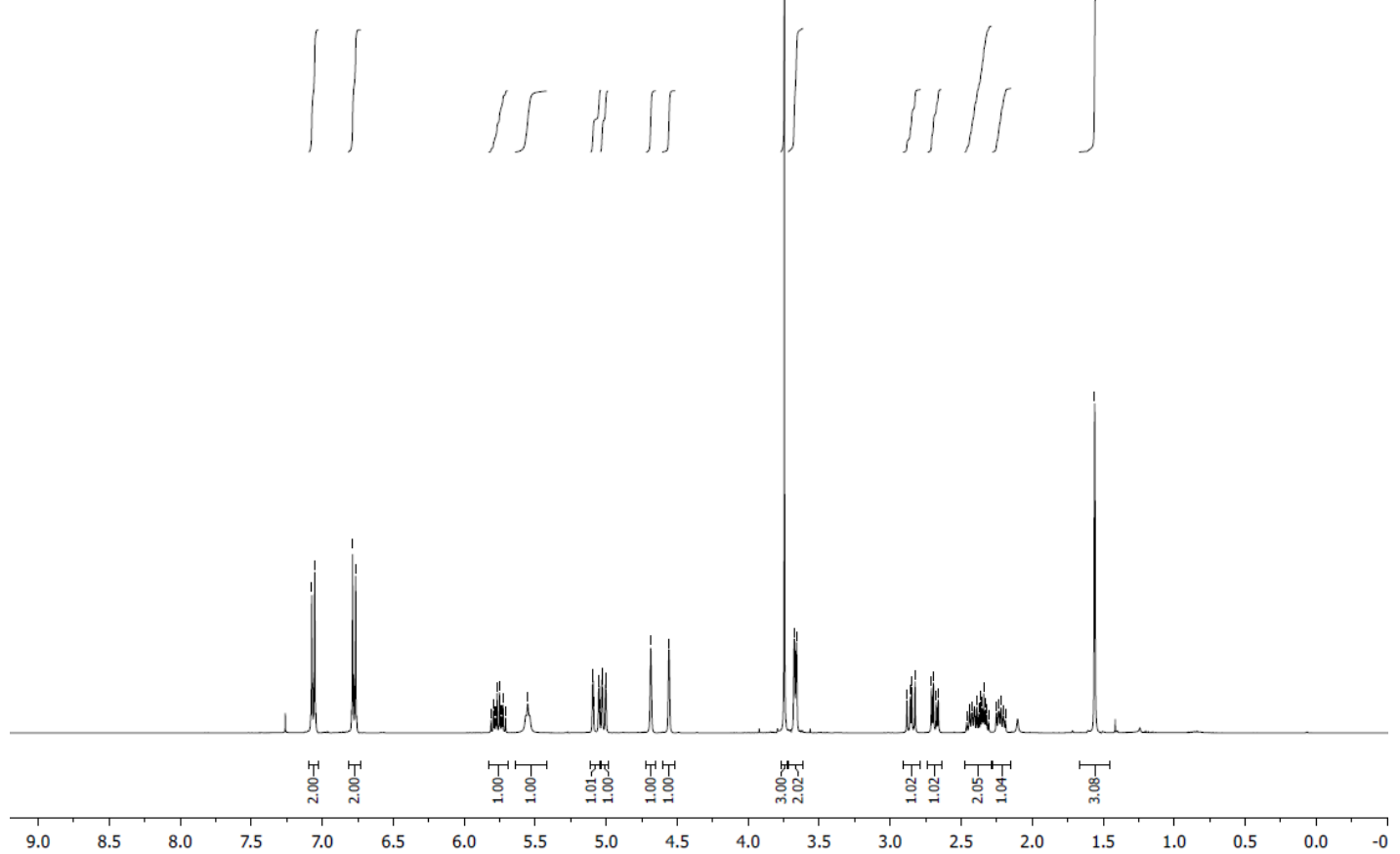

${ }^{13} \mathrm{C}-\mathrm{NMR}$ of compound 9c (101 $\left.\mathrm{MHz}, \mathrm{CDCl}_{3}\right)$

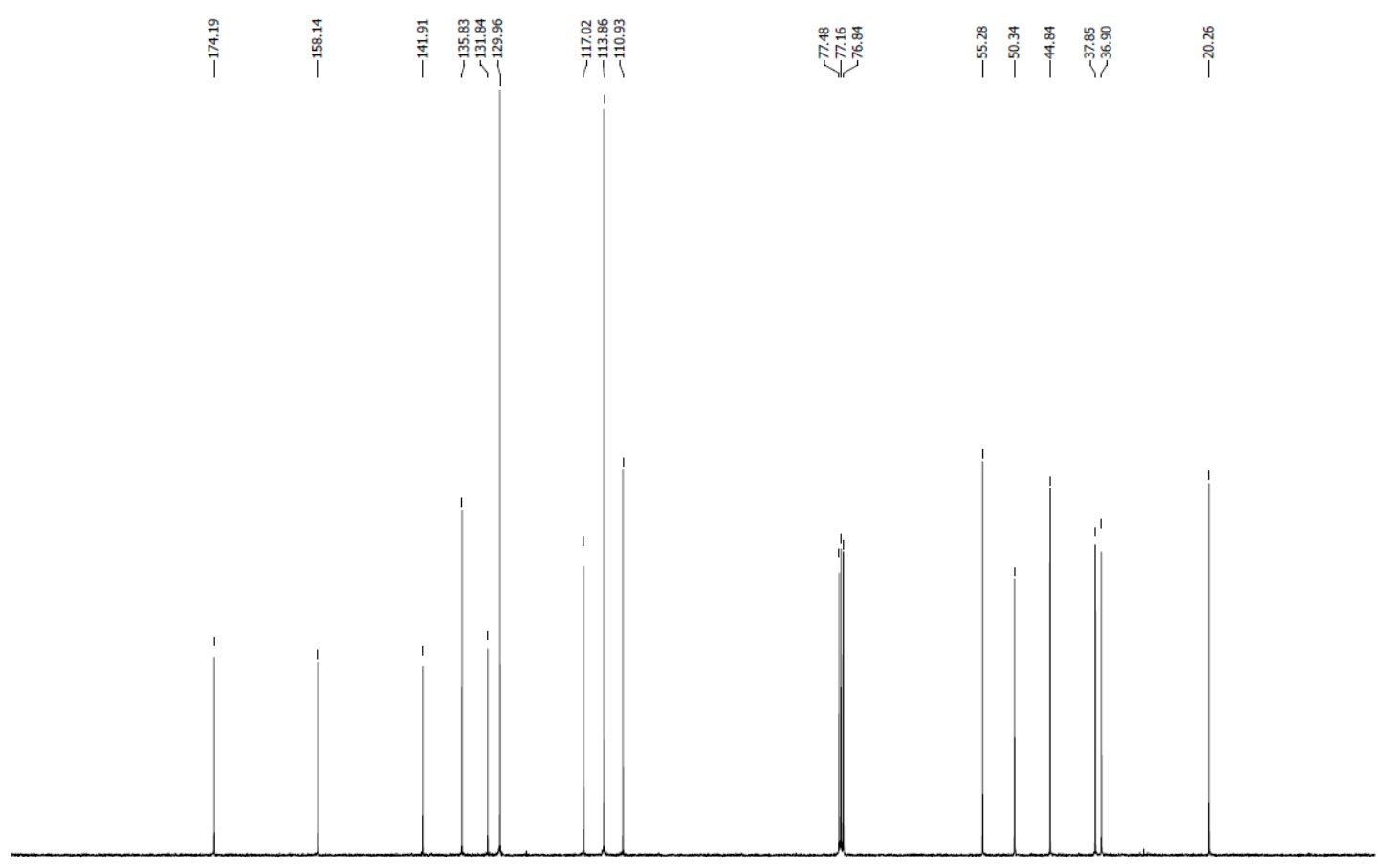

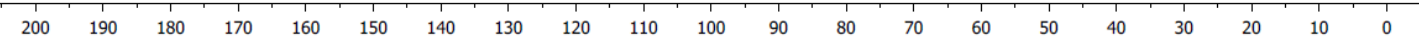




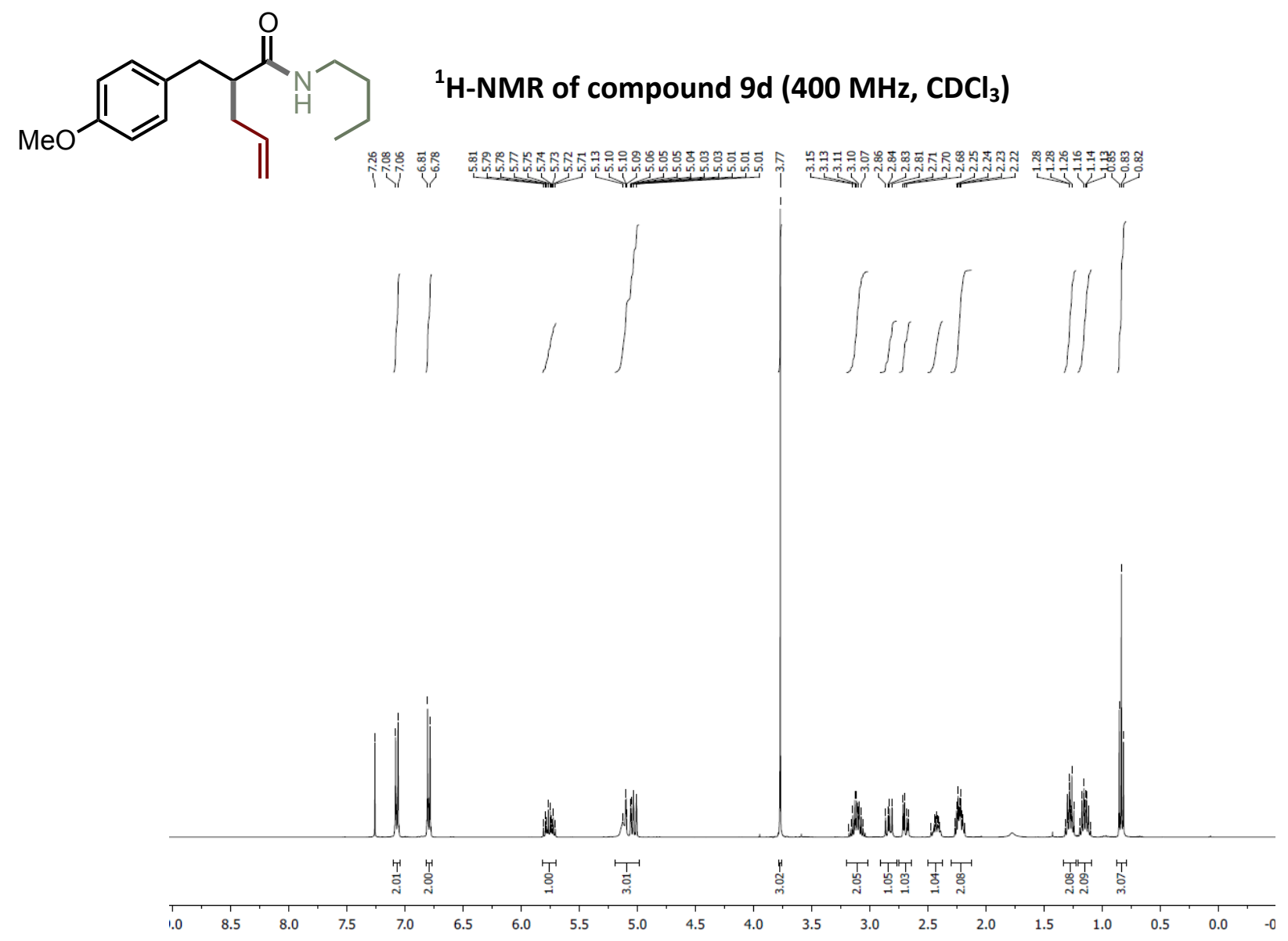

${ }^{13} \mathrm{C}-\mathrm{NMR}$ of compound 9d (101 $\mathrm{MHz}, \mathrm{CDCl}_{3}$ )

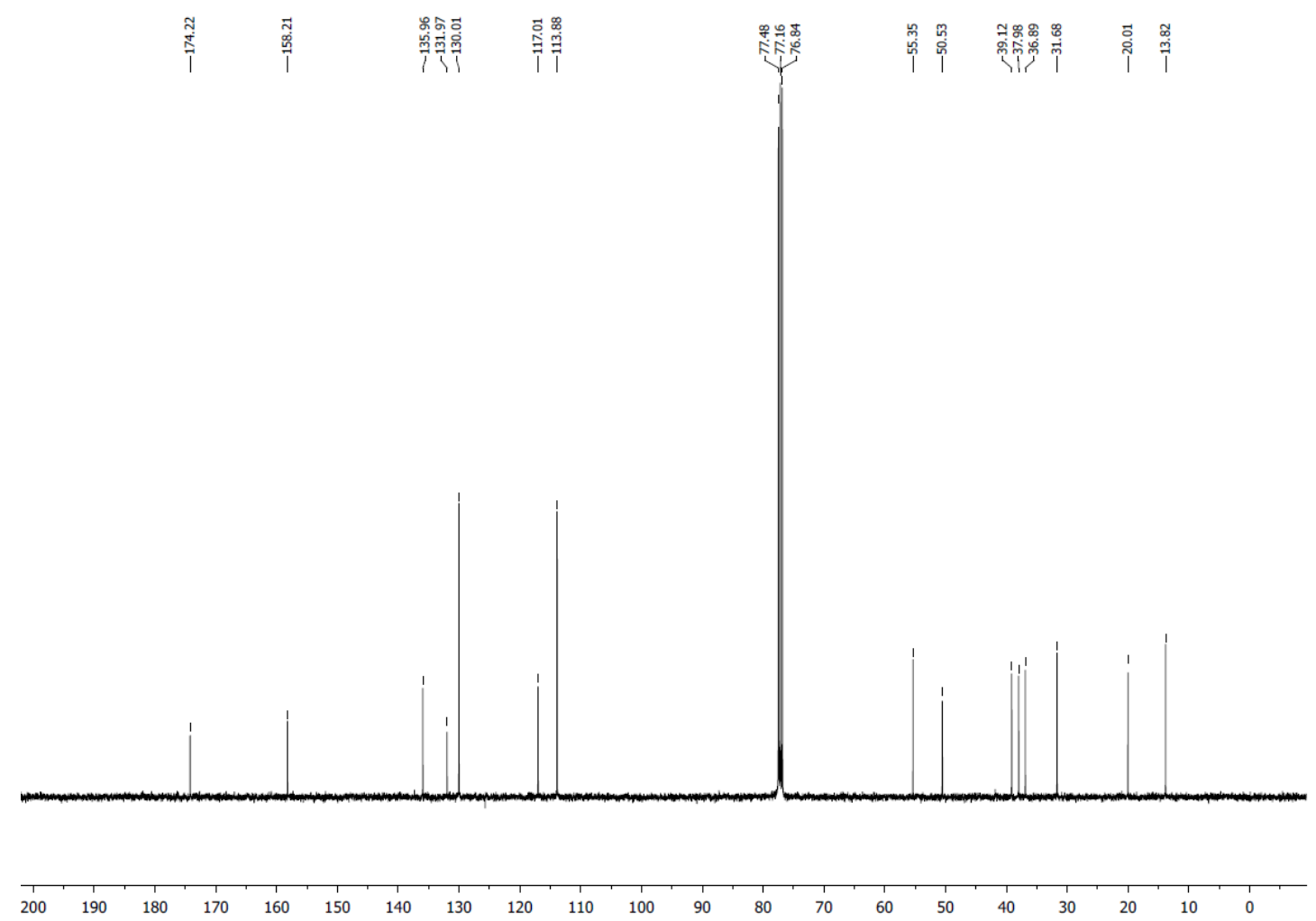


${ }^{1} \mathrm{H}-\mathrm{NMR}$ of compound $9 \mathrm{e}\left(400 \mathrm{MHz}, \mathrm{CDCl}_{3}\right)$

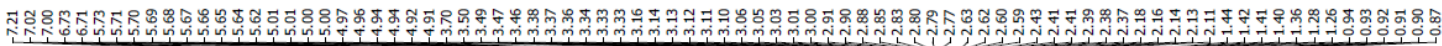<smiles>C=CCC(Cc1ccc(OC)cc1)C(=O)N1CCCCC1</smiles>
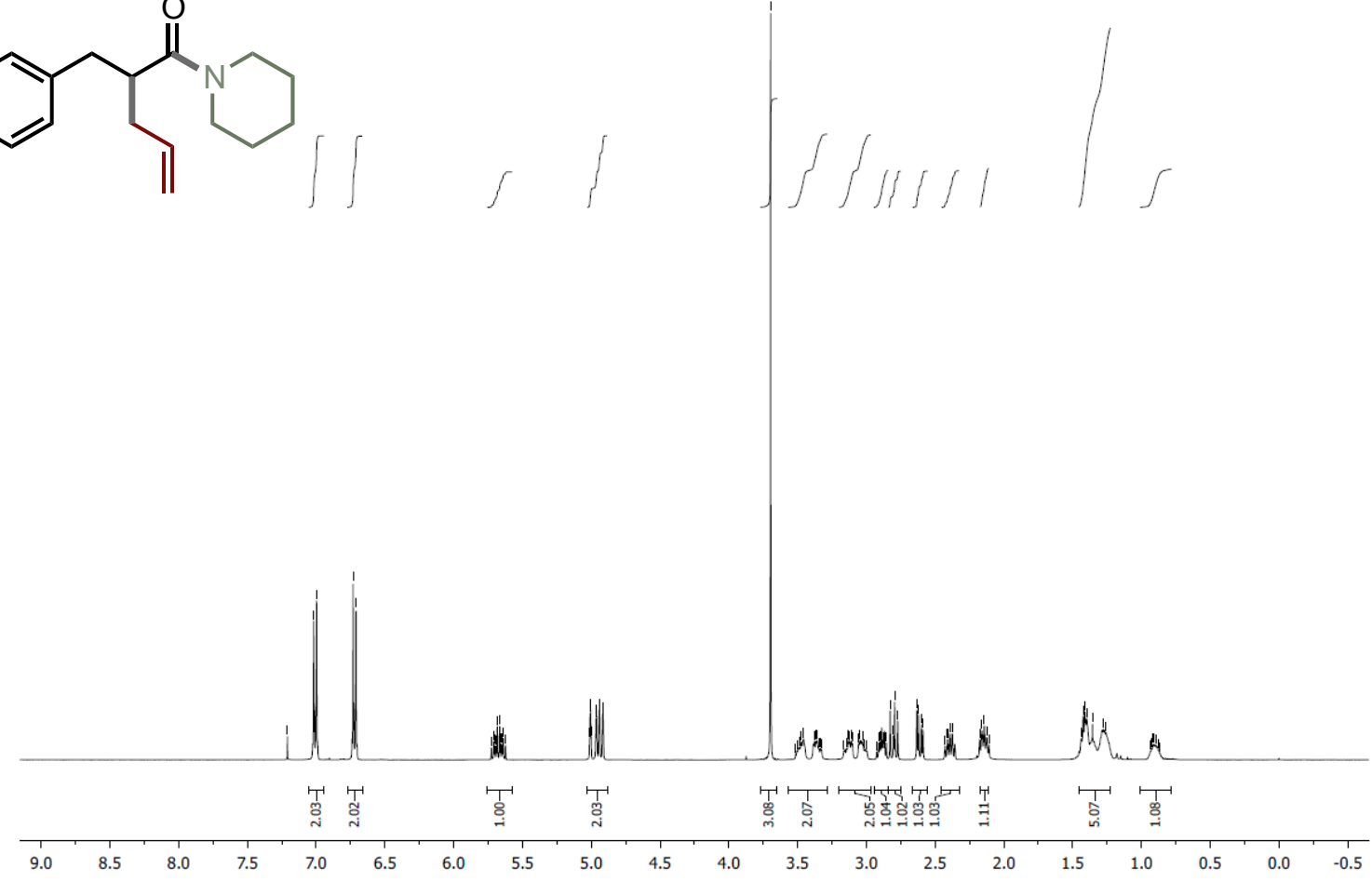

${ }^{13} \mathrm{C}-\mathrm{NMR}$ of compound $9 \mathrm{e}\left(101 \mathrm{MHz}, \mathrm{CDCl}_{3}\right)$

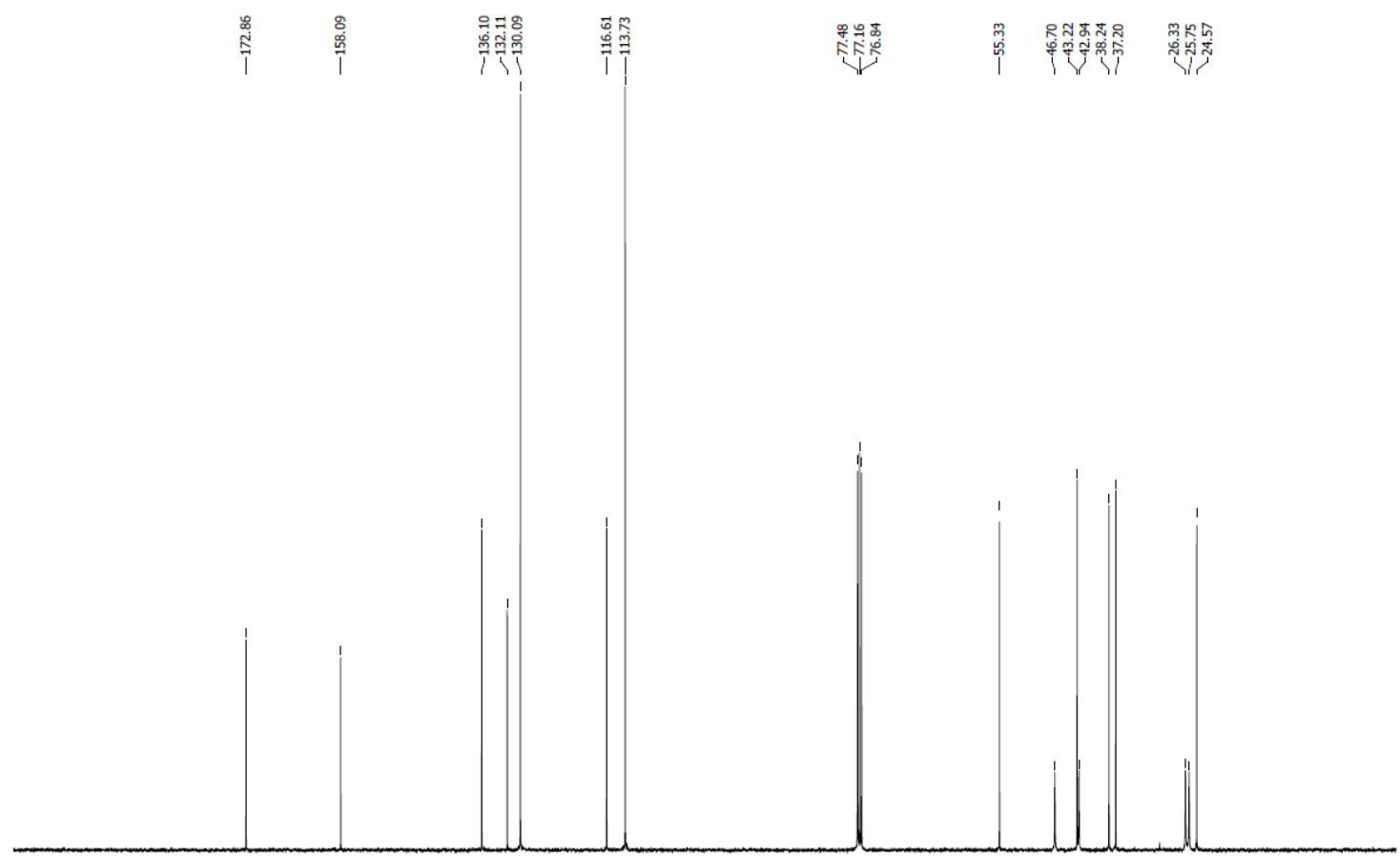




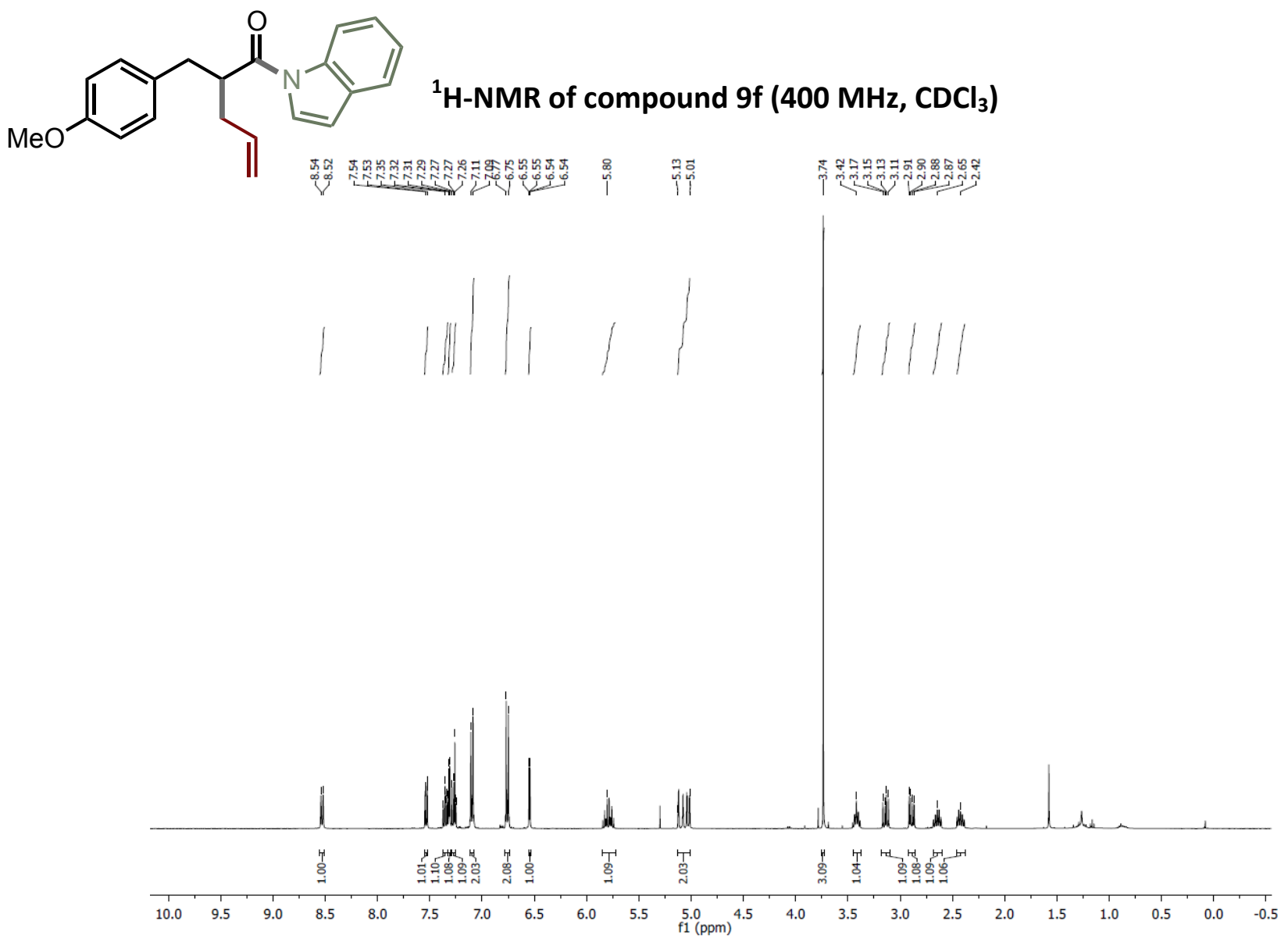

${ }^{13} \mathrm{C}-\mathrm{NMR}$ of compound $9 \mathrm{f}\left(101 \mathrm{MHz}, \mathrm{CDCl}_{3}\right)$
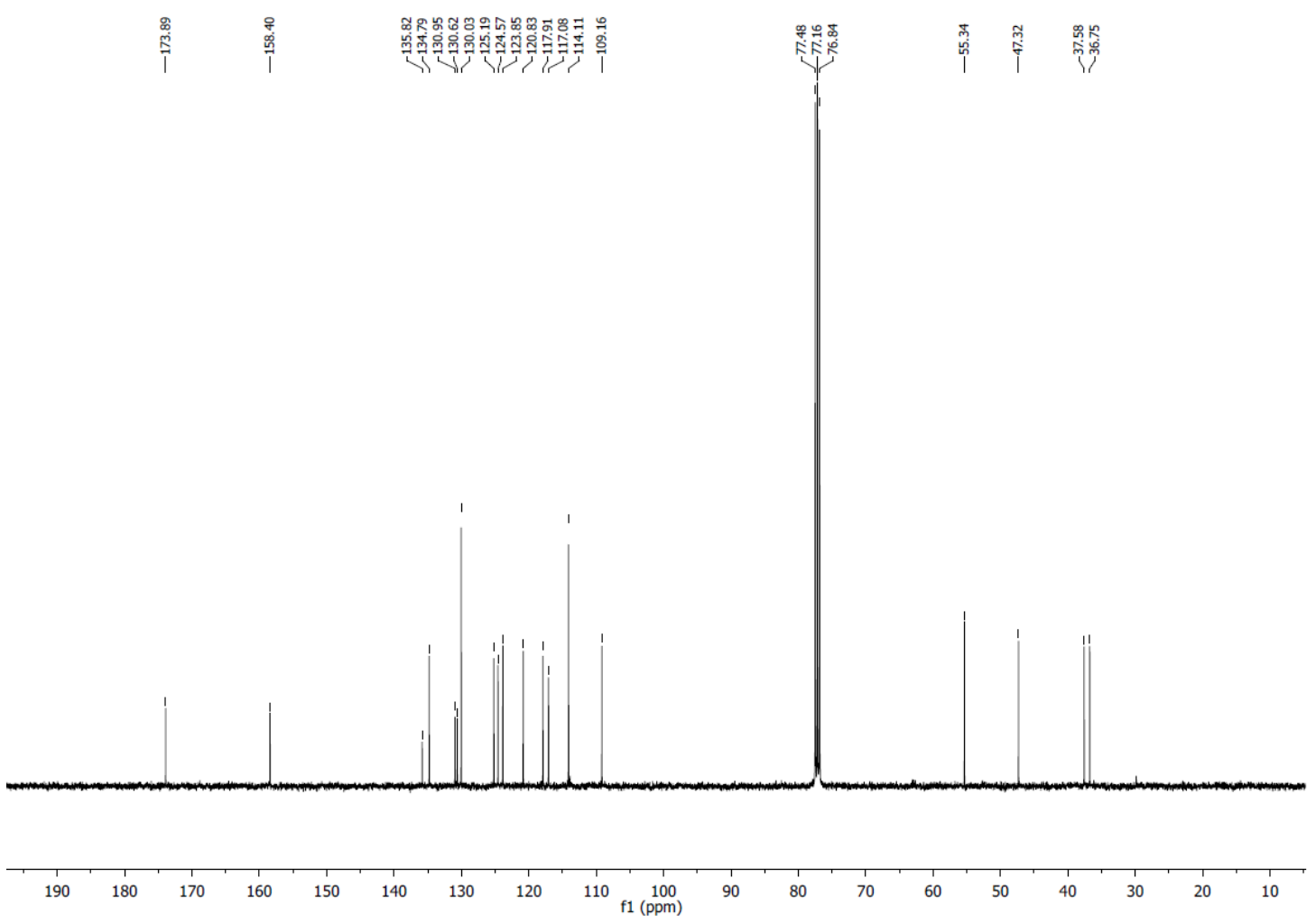

S35 


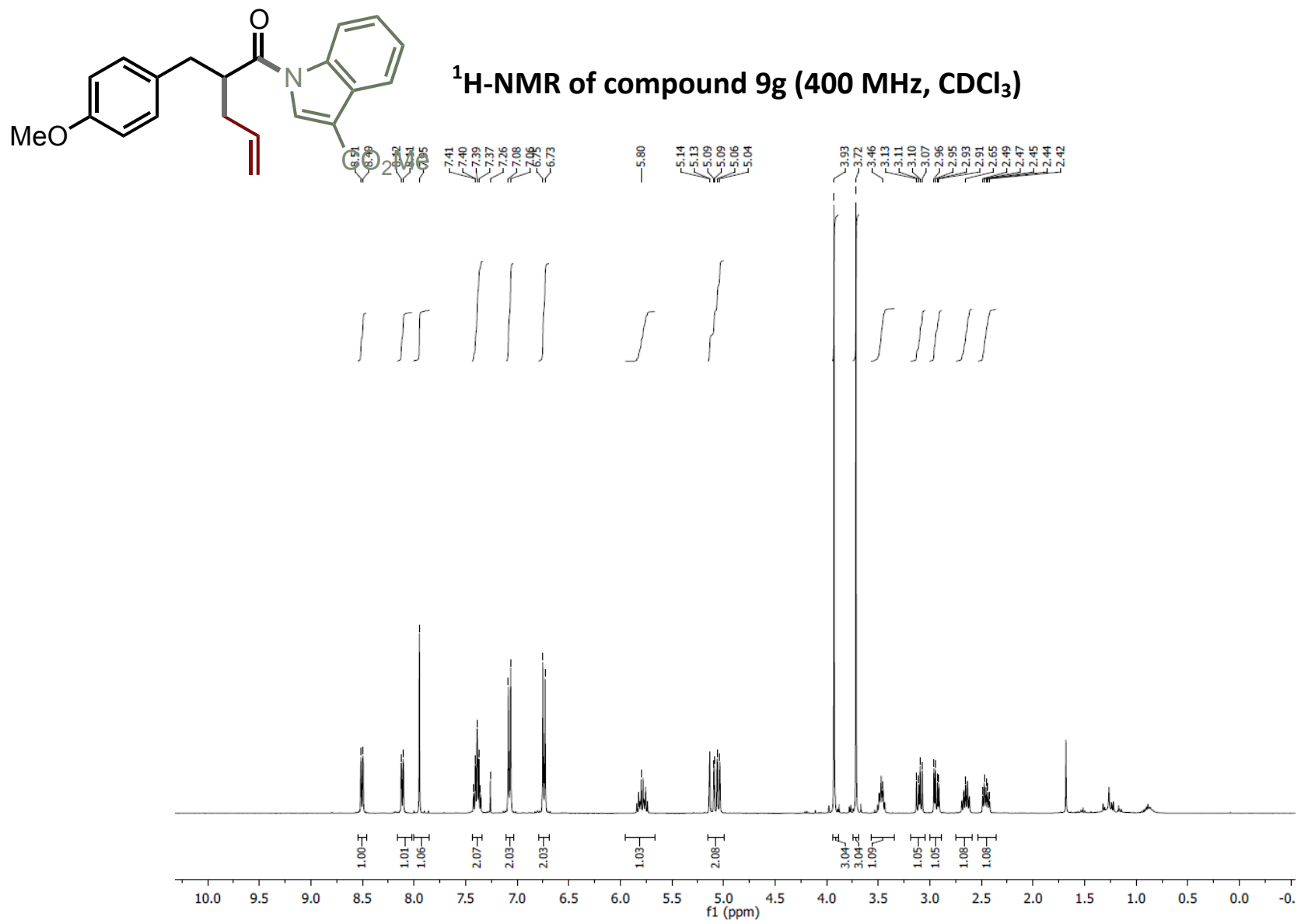

${ }^{13} \mathrm{C}-\mathrm{NMR}$ of compound $9 \mathrm{~g}\left(101 \mathrm{MHz}, \mathrm{CDCl}_{3}\right)$

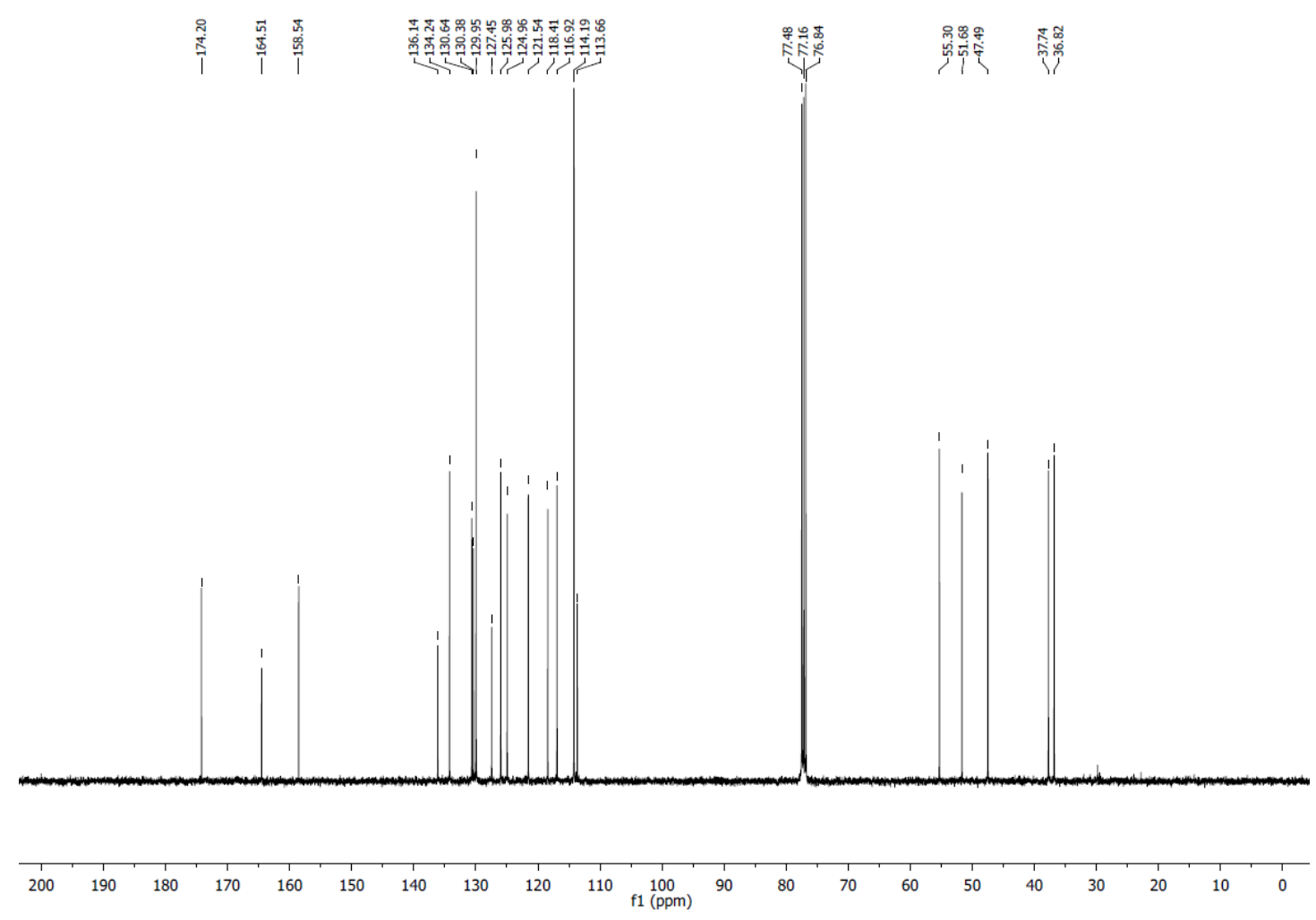




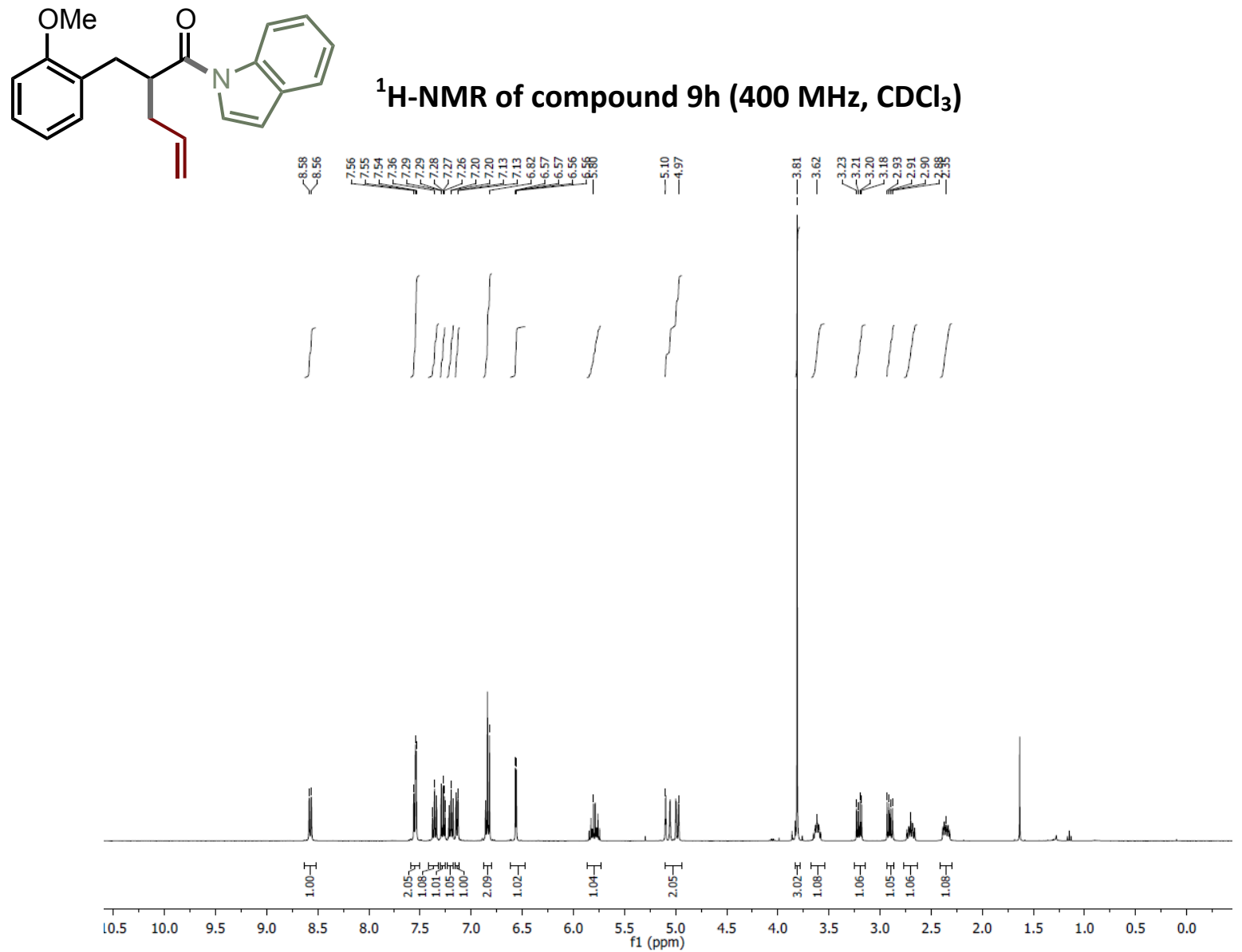

${ }^{13} \mathrm{C}-\mathrm{NMR}$ of compound 9h (101 $\mathrm{MHz}, \mathrm{CDCl}_{3}$ )

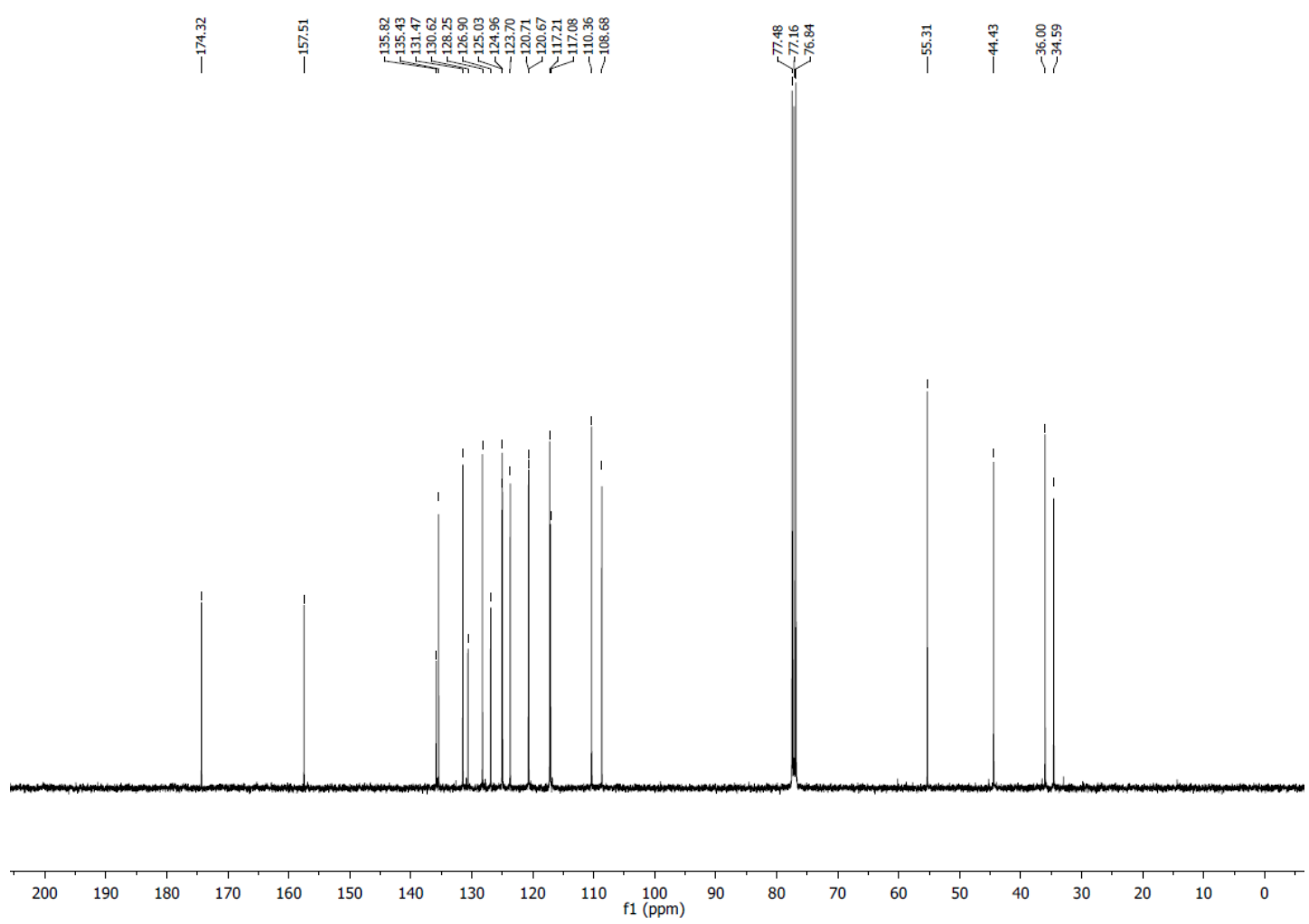


on

${ }^{1} \mathrm{H}-\mathrm{NMR}$ of compound $9 \mathrm{i}\left(400 \mathrm{MHz}, \mathrm{CDCl}_{3}\right)$

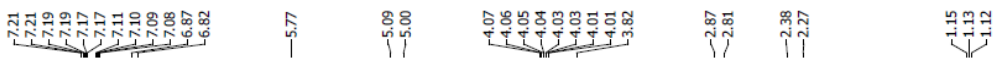
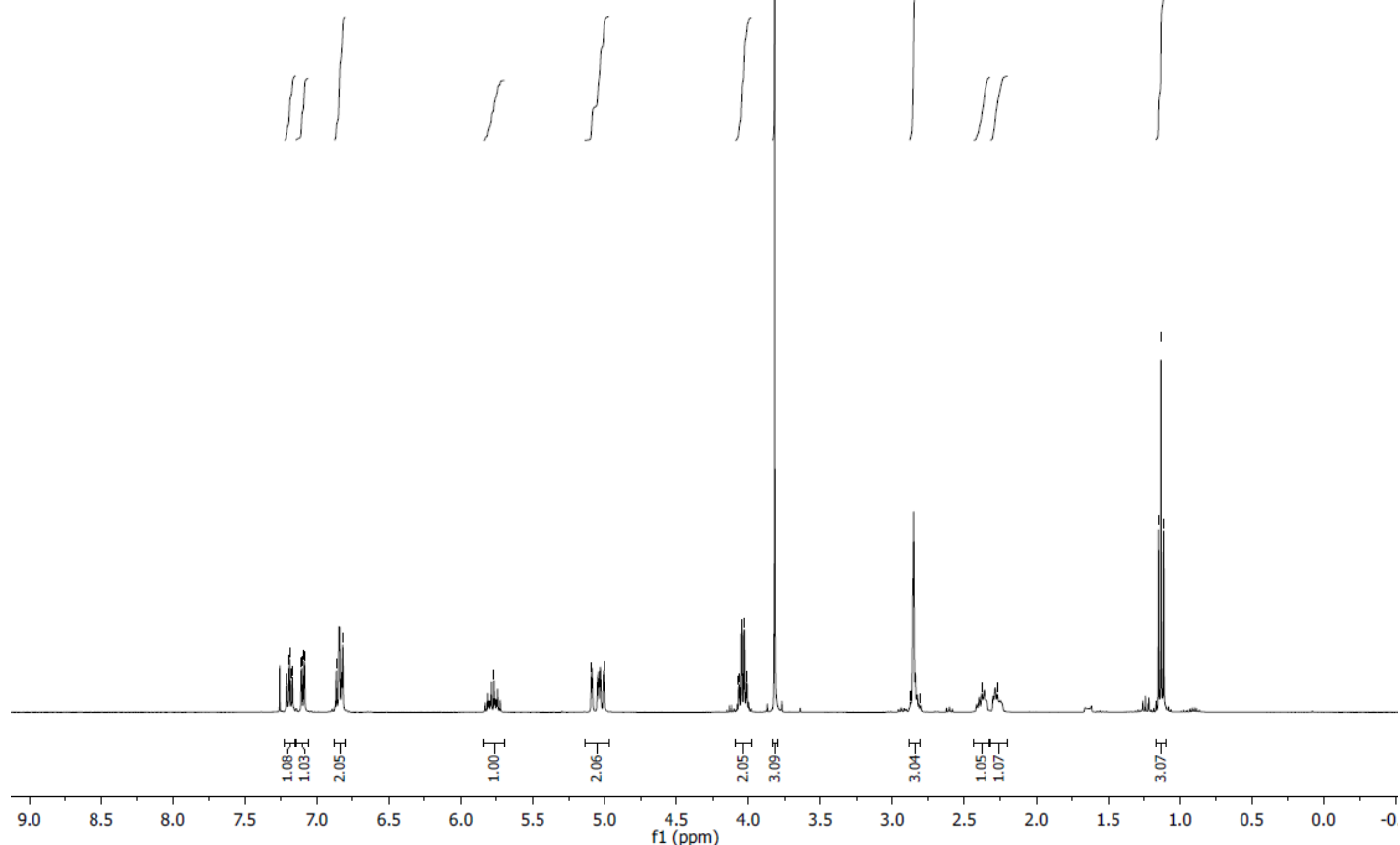

${ }^{13} \mathrm{C}-\mathrm{NMR}$ of compound $9 \mathrm{i}\left(101 \mathrm{MHz}, \mathrm{CDCl}_{3}\right)$

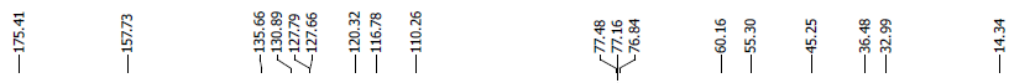

$\begin{array}{llllllllllllllllllllllllllllllll}210 & 200 & 190 & 180 & 170 & 160 & 150 & 140 & 130 & 120 & 110 & 100 & 90 & 80 & 70 & 60 & 50 & 40 & 30 & 20 & 10 & 0 & -10\end{array}$ 


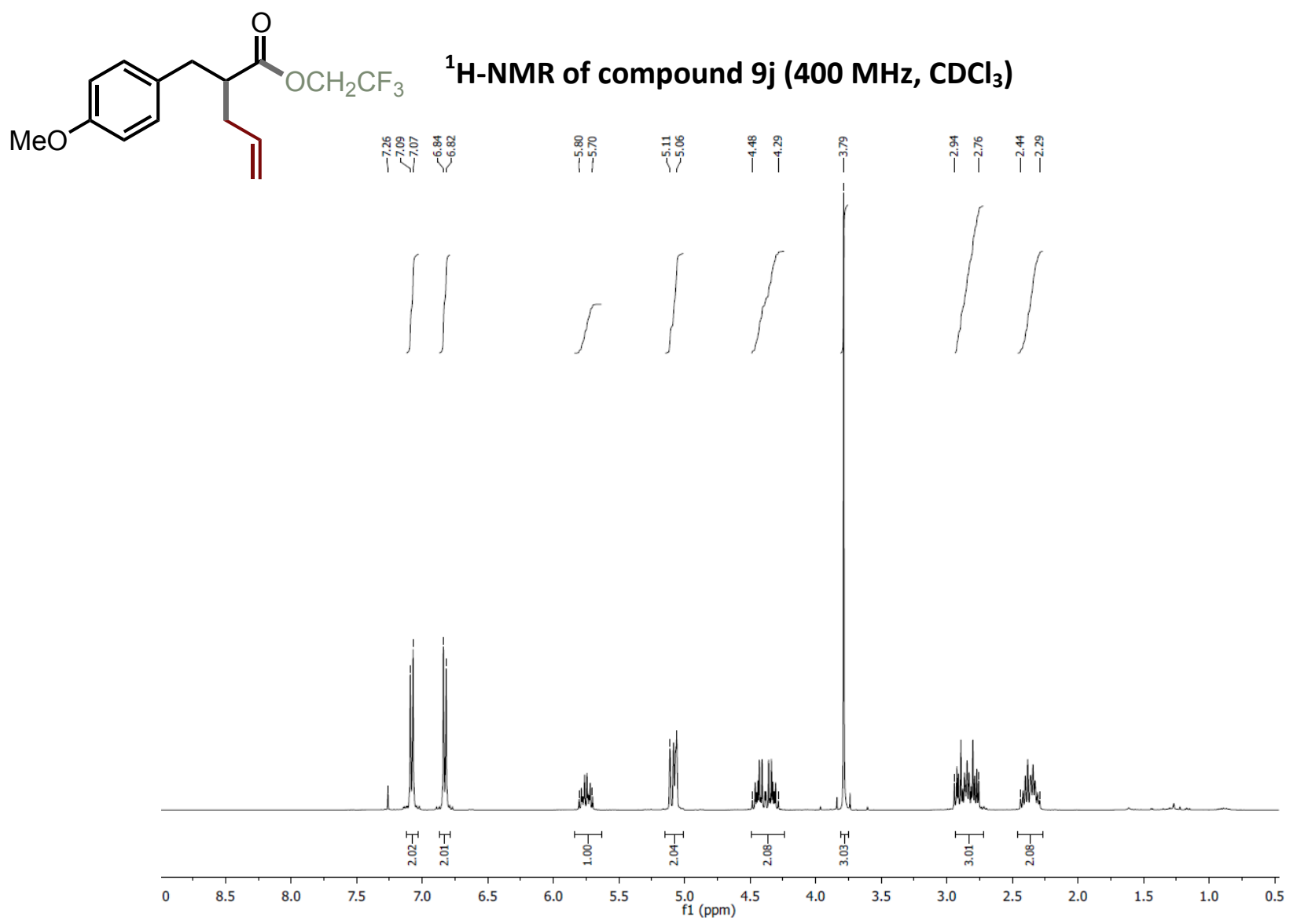

${ }^{13} \mathrm{C}-\mathrm{NMR}$ of compound 9j (101 $\left.\mathrm{MHz}, \mathrm{CDCl}_{3}\right)$

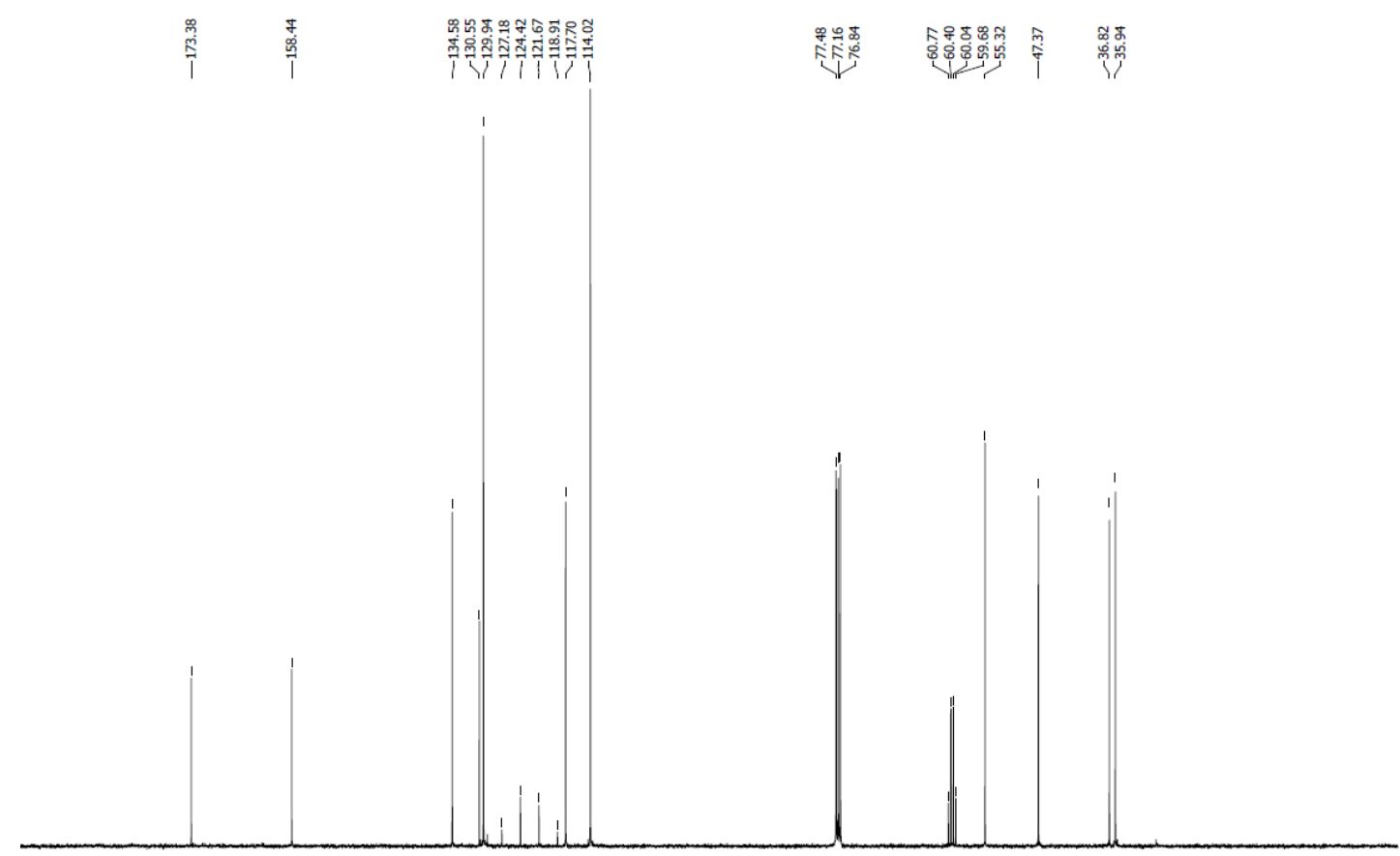

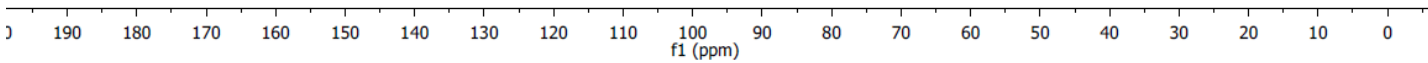


<smiles>C=CCC(Cc1ccc(OC)cc1)C(=O)[Si]CC</smiles>

${ }^{1} \mathrm{H}-\mathrm{NMR}$ of compound $9 \mathrm{k}\left(400 \mathrm{MHz}, \mathrm{CDCl}_{3}\right)$

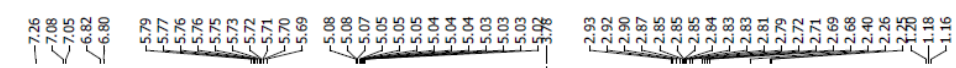

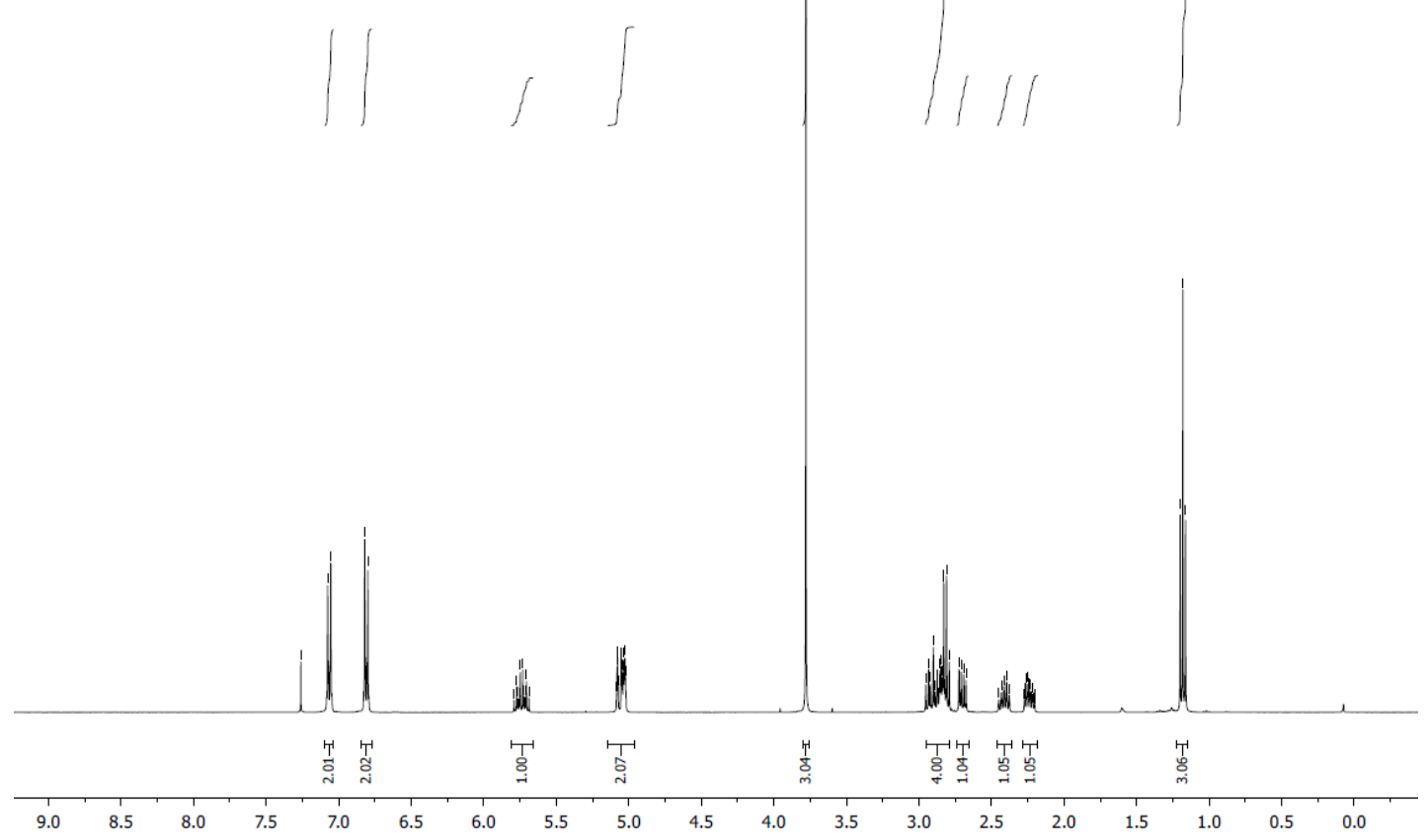

${ }^{13} \mathrm{C}-\mathrm{NMR}$ of compound 9k (101 $\left.\mathrm{MHz}, \mathrm{CDCl}_{3}\right)$

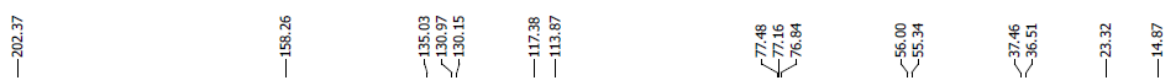

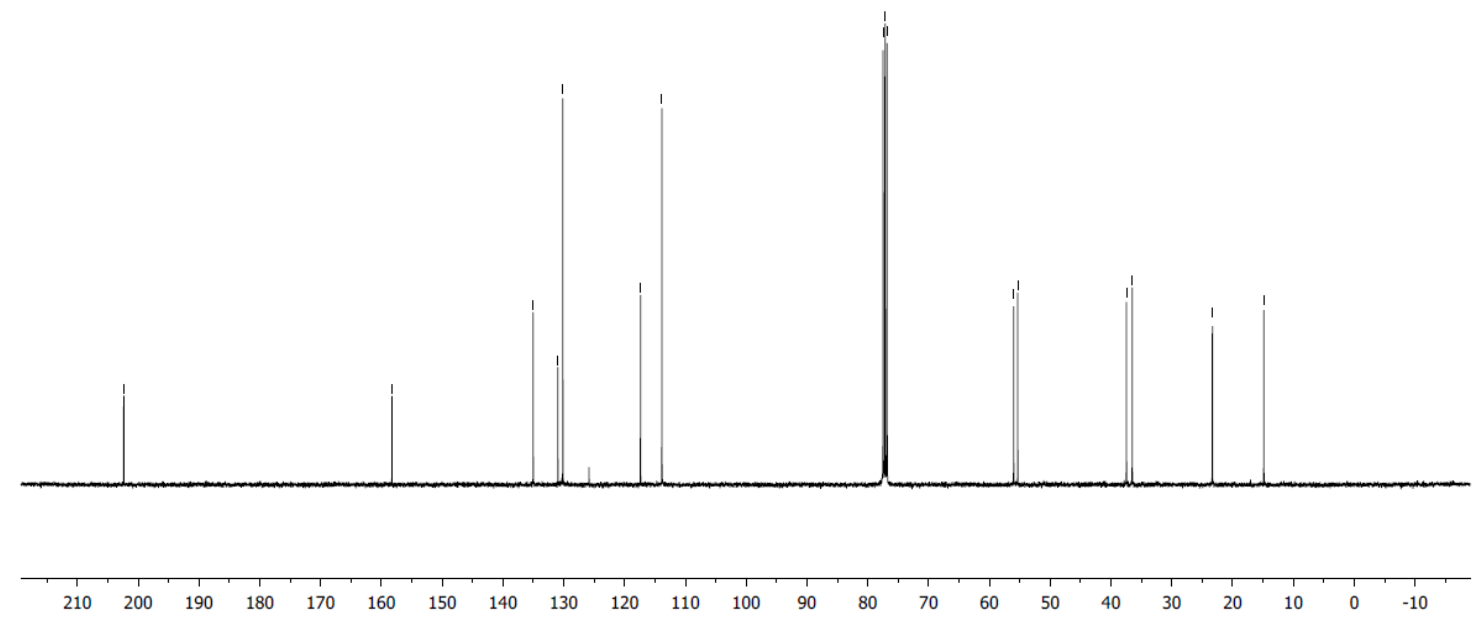




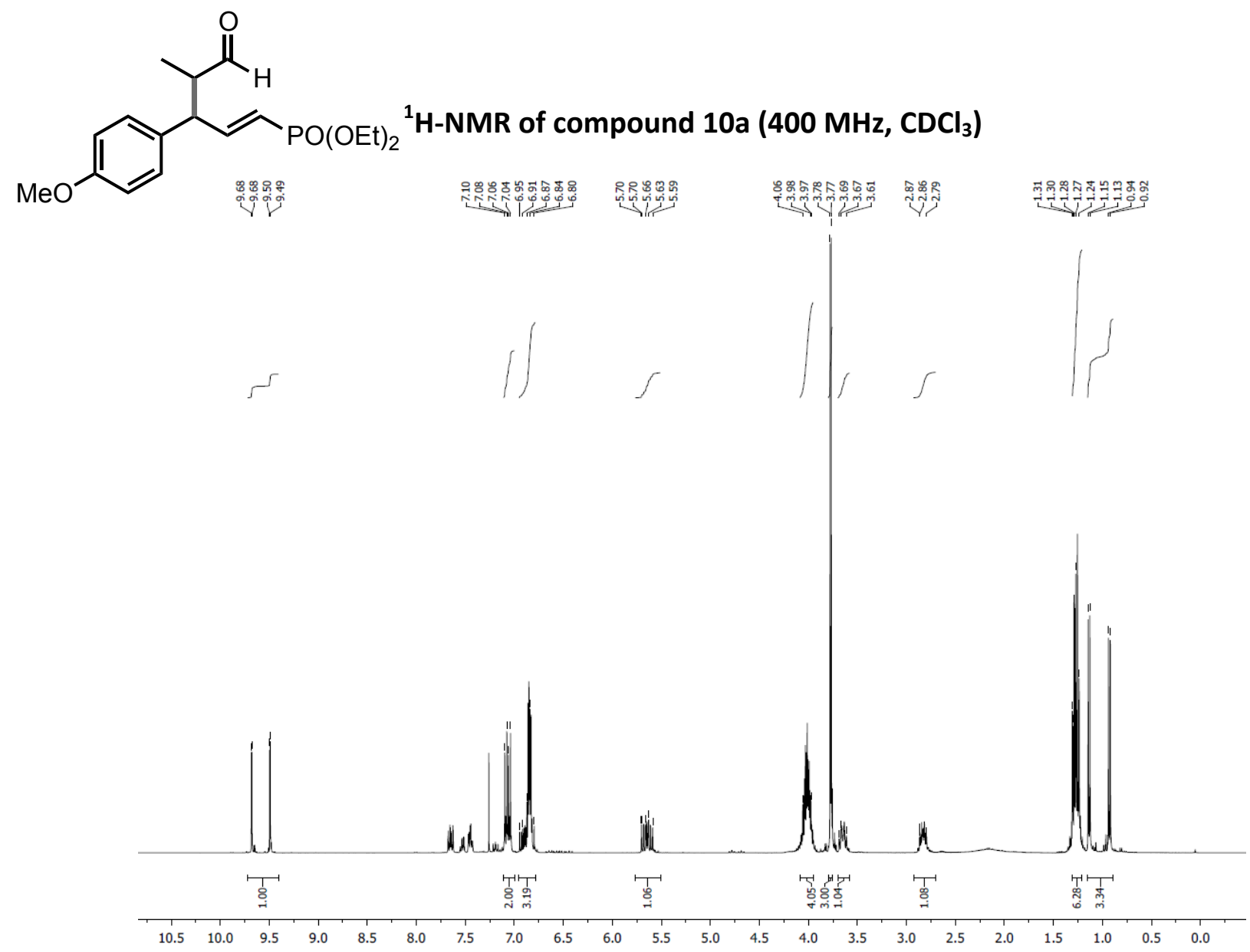

${ }^{13} \mathrm{C}-\mathrm{NMR}$ of compound 10a (101 $\mathrm{MHz}, \mathrm{CDCl}_{3}$ )

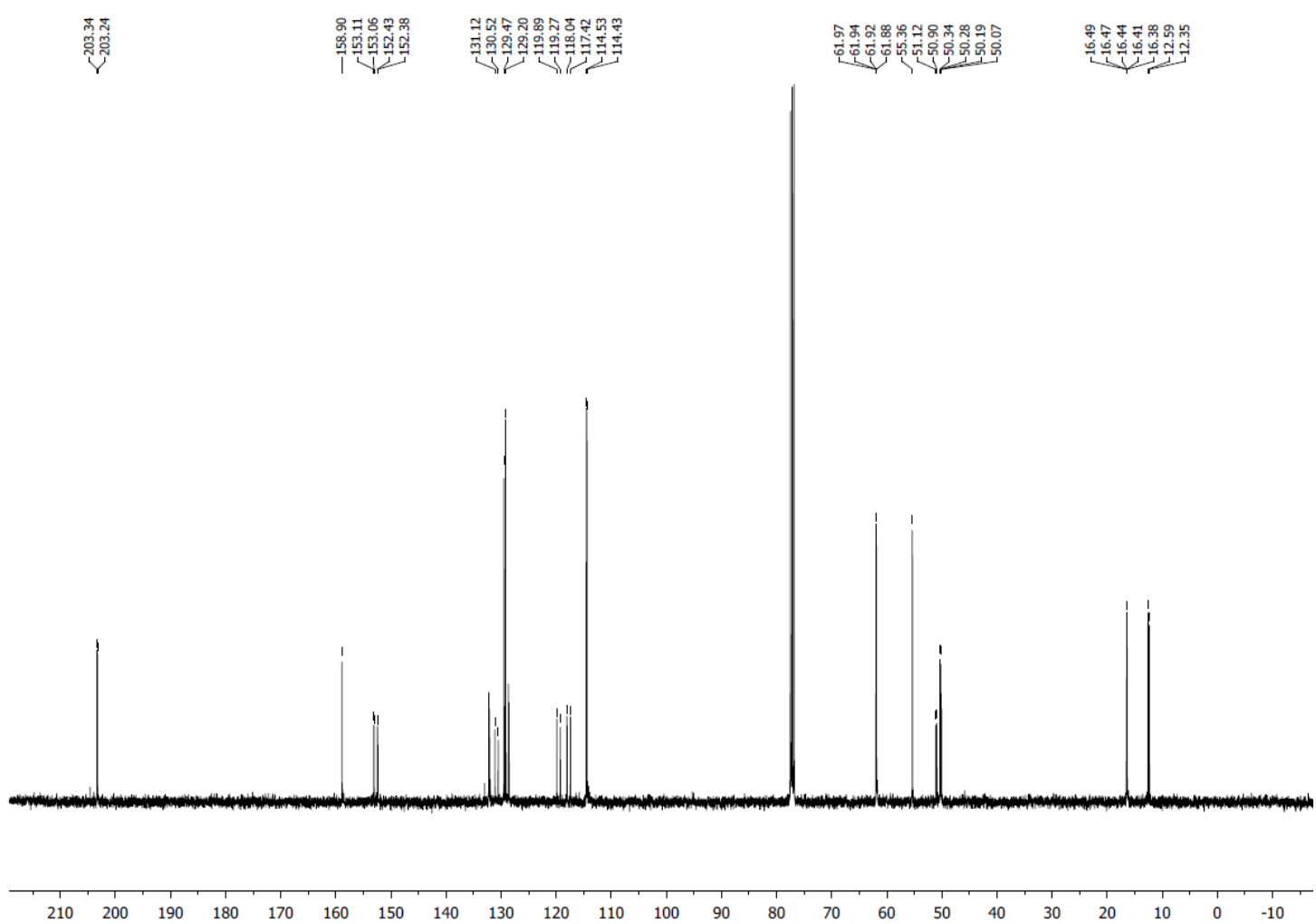




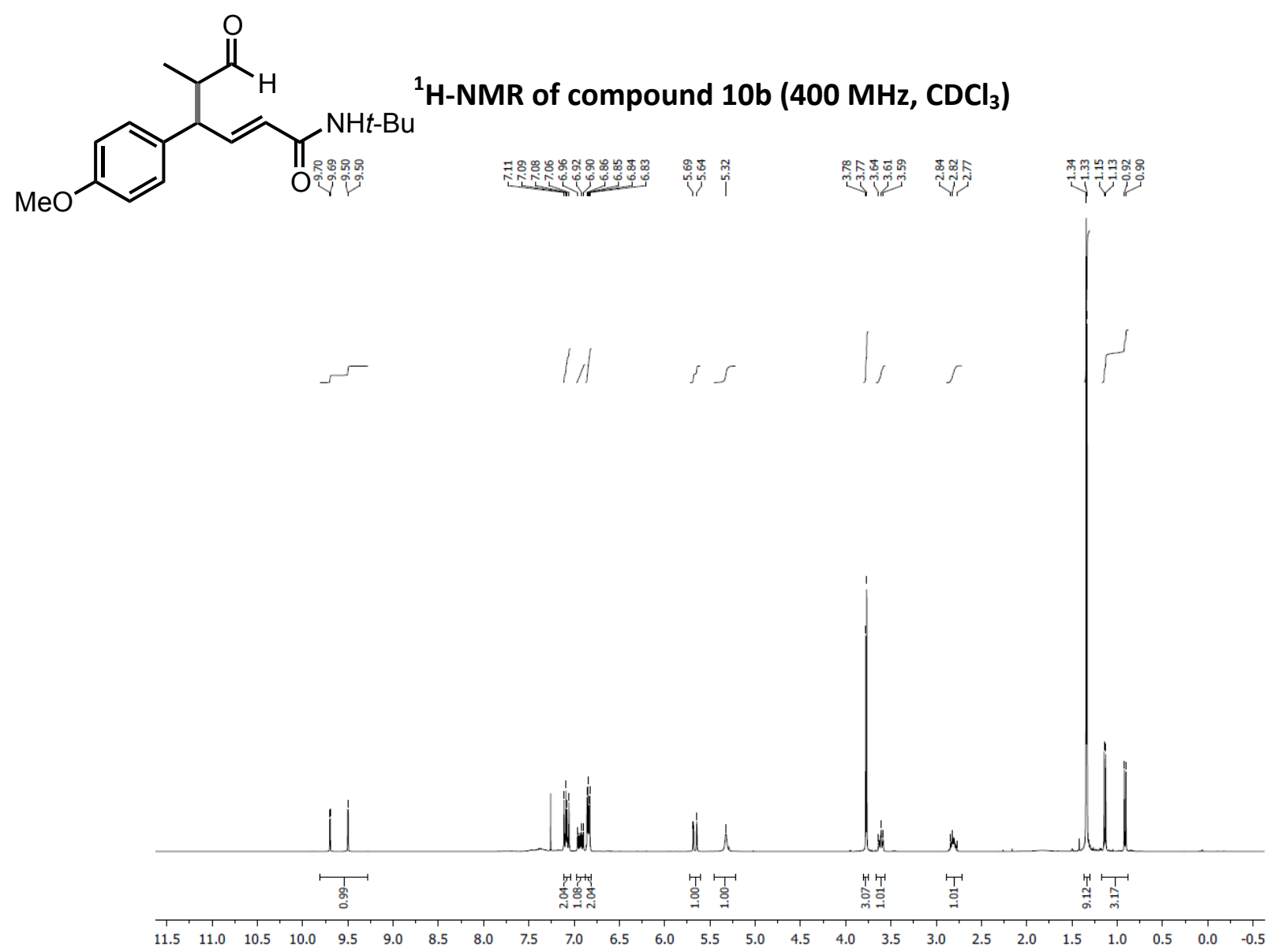

${ }^{13} \mathrm{C}-\mathrm{NMR}$ of compound $10 \mathrm{~b}\left(101 \mathrm{MHz}^{\mathrm{CDCl}}\right)_{3}$

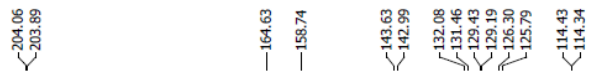

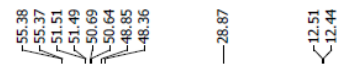

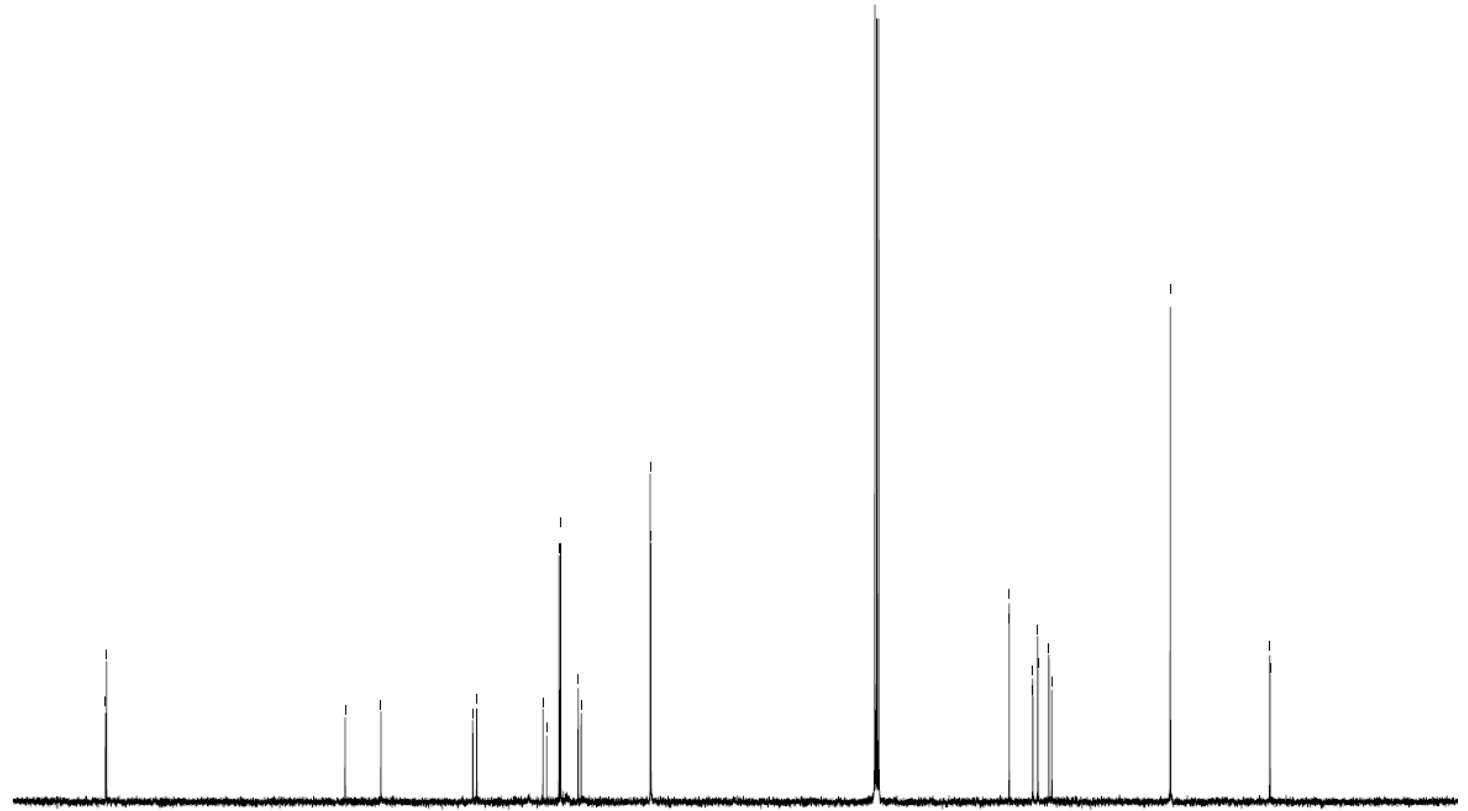

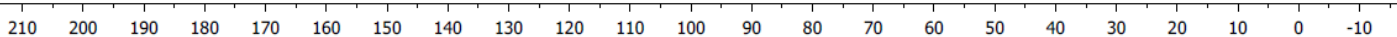




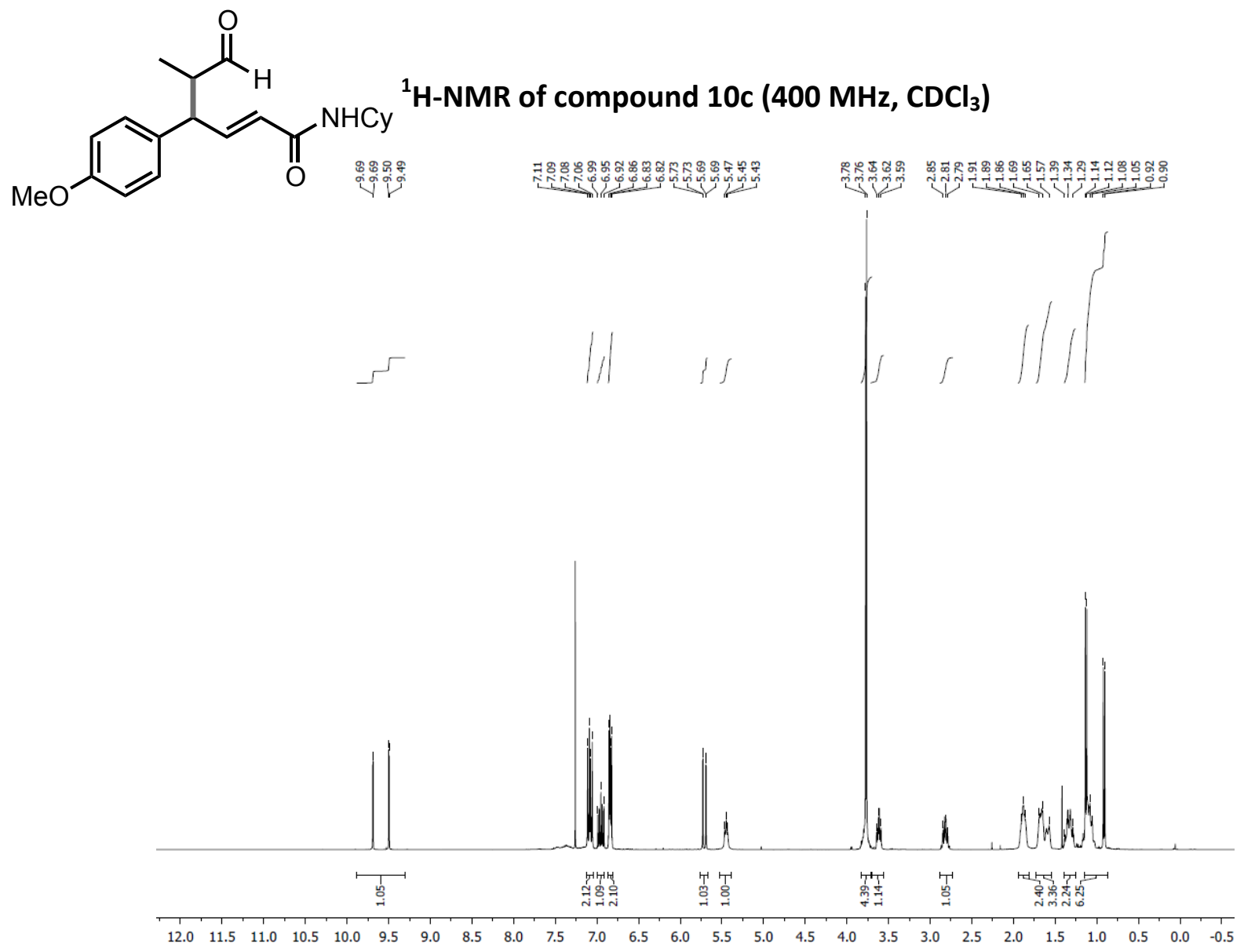

${ }^{13} \mathrm{C}-\mathrm{NMR}$ of compound $10 \mathrm{c}\left(101 \mathrm{MHz}, \mathrm{CDCl}_{3}\right)$

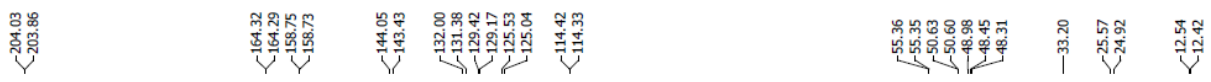

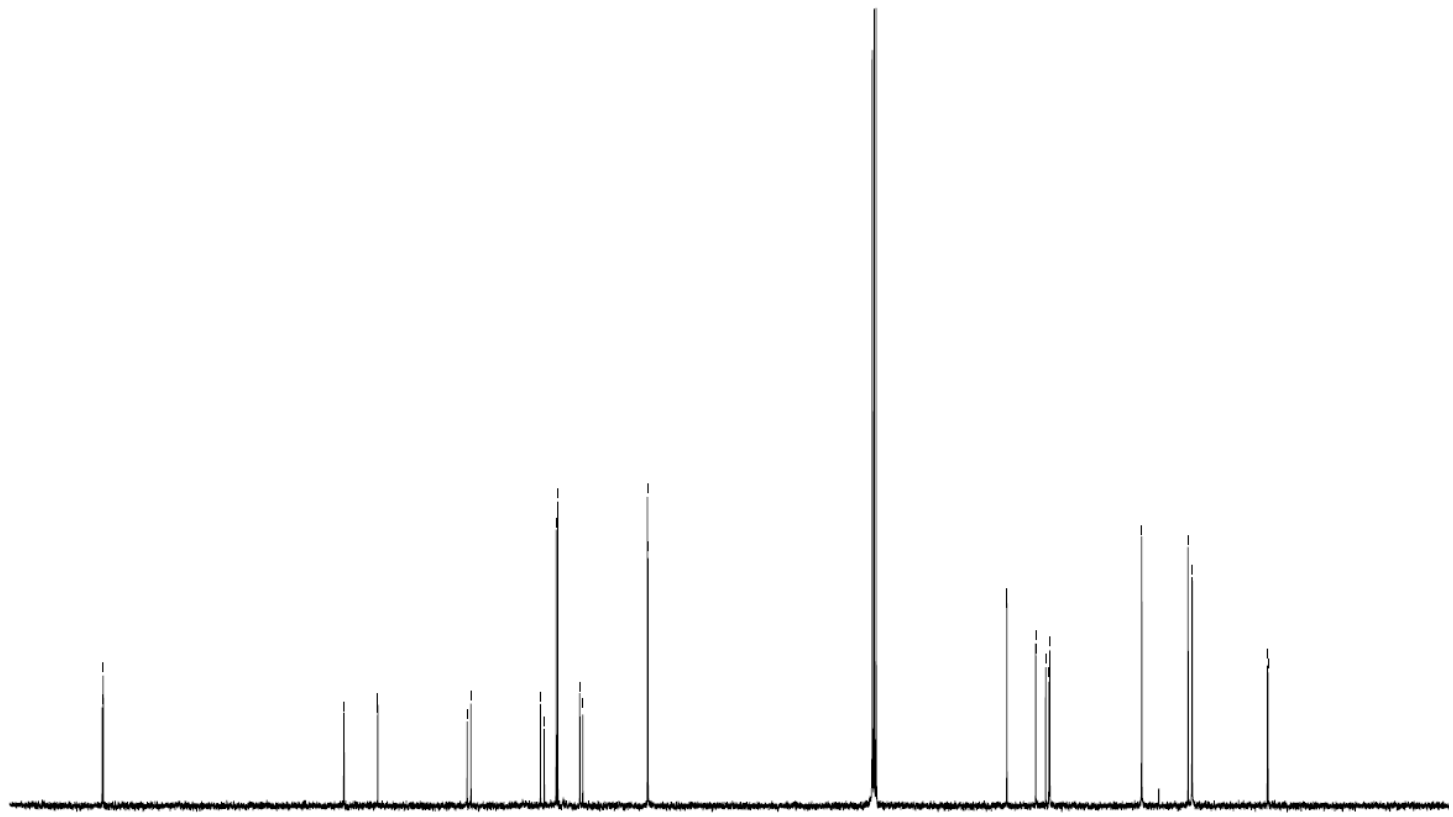

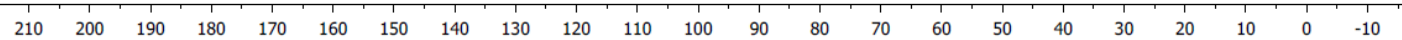


<smiles>CCCCCCC(C)C(C=CC(=O)NC(C)(C)C)c1ccccc1</smiles>

${ }^{1} \mathrm{H}-\mathrm{NMR}$ of compound $10 \mathrm{~d}\left(400 \mathrm{MHz}, \mathrm{CDCl}_{3}\right.$ )

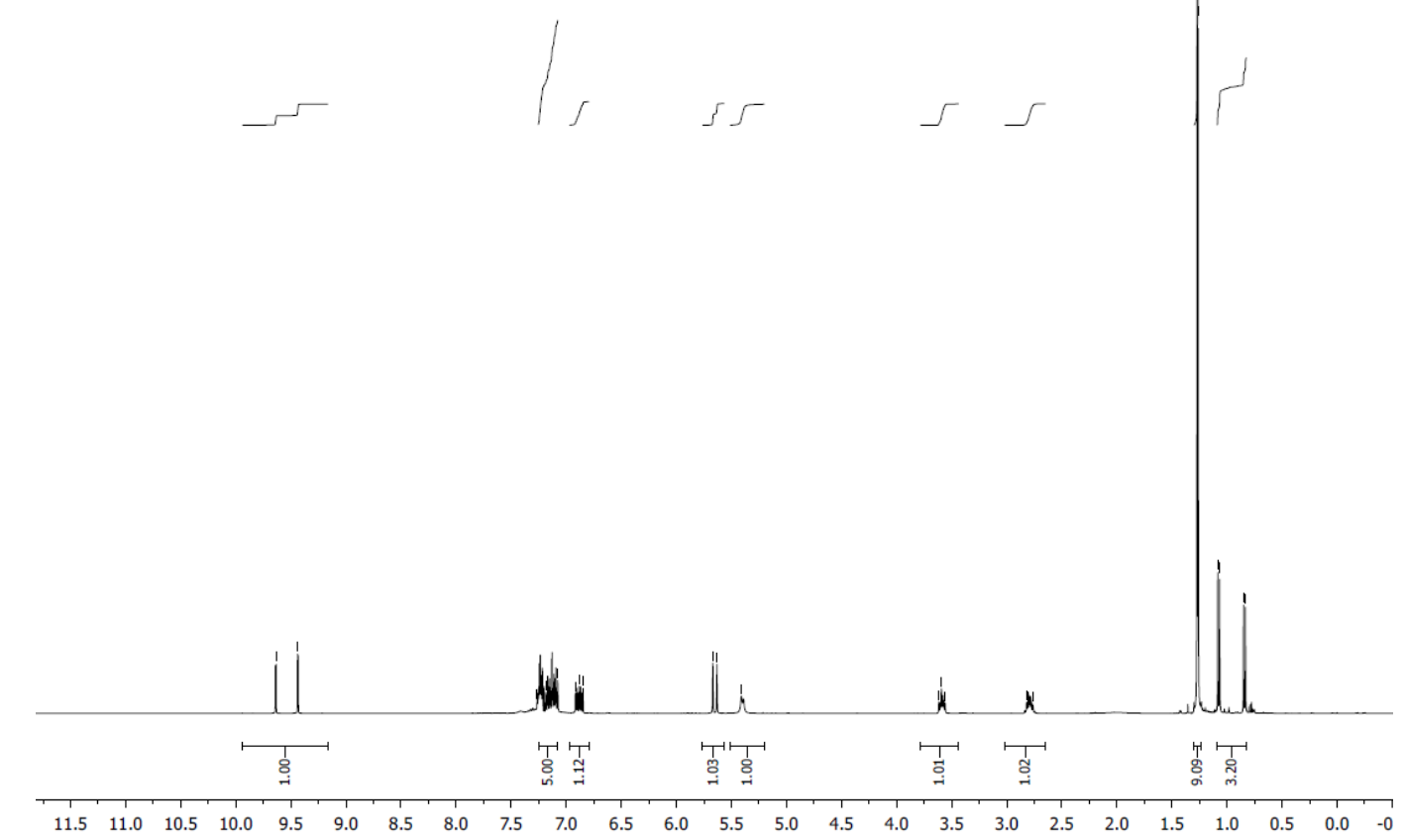

${ }^{13} \mathrm{C}-\mathrm{NMR}$ of compound $10 \mathrm{~d}\left(101 \mathrm{MHz}, \mathrm{CDCl}_{3}\right)$

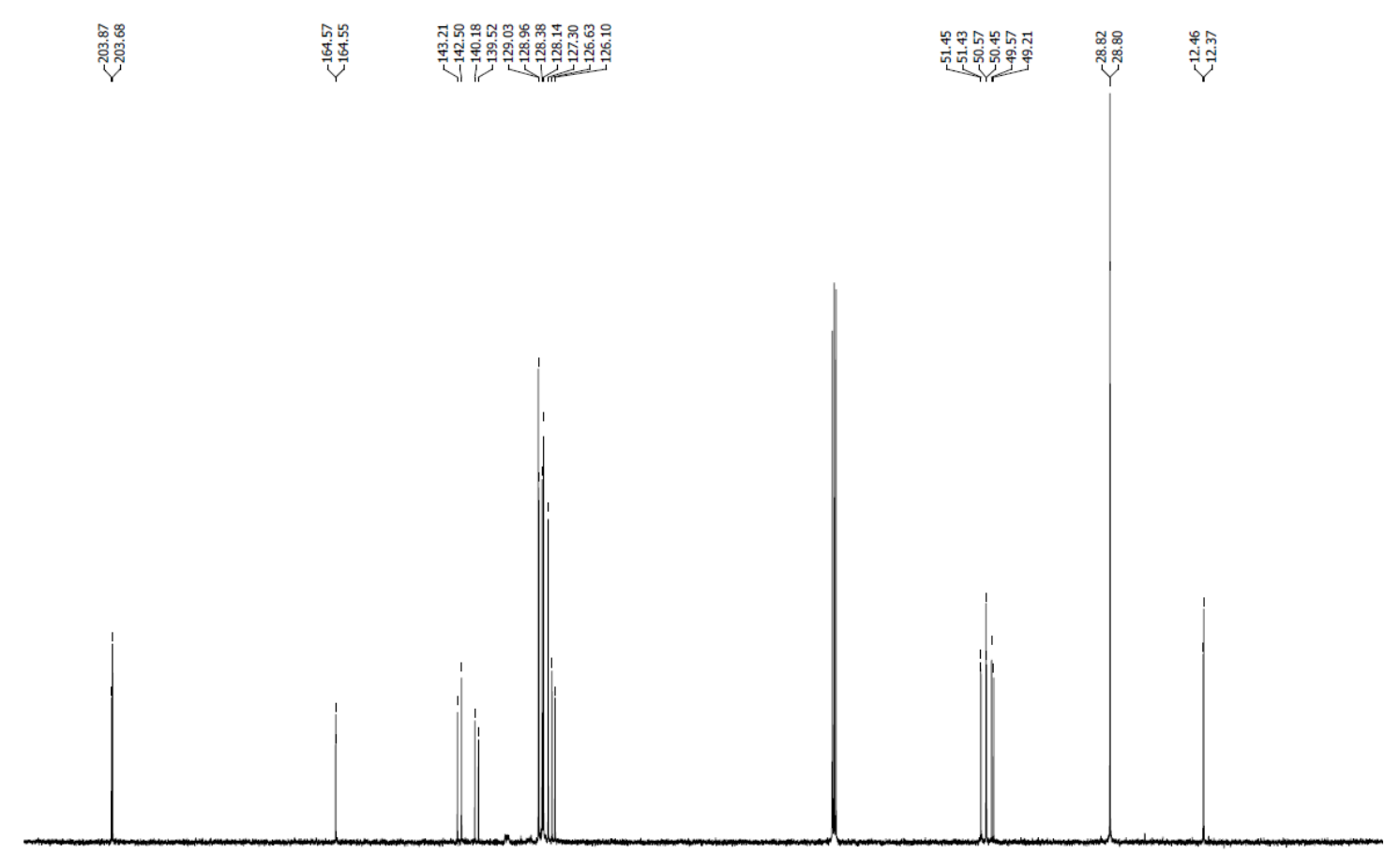

\begin{tabular}{|llllllllllllllllllllllll}
1 & 1 & 200 & 190 & 180 & 170 & 160 & 150 & 140 & 130 & 120 & 110 & 100 & 90 & 80 & 70 & 60 & 50 & 40 & 30 & 20 & 10 & 0 & -10
\end{tabular} 


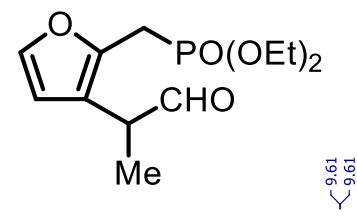

${ }^{1} \mathrm{H}-\mathrm{NMR}$ of compound $11 \mathrm{a}\left(400 \mathrm{MHz}, \mathrm{CDCl}_{3}\right)$

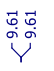

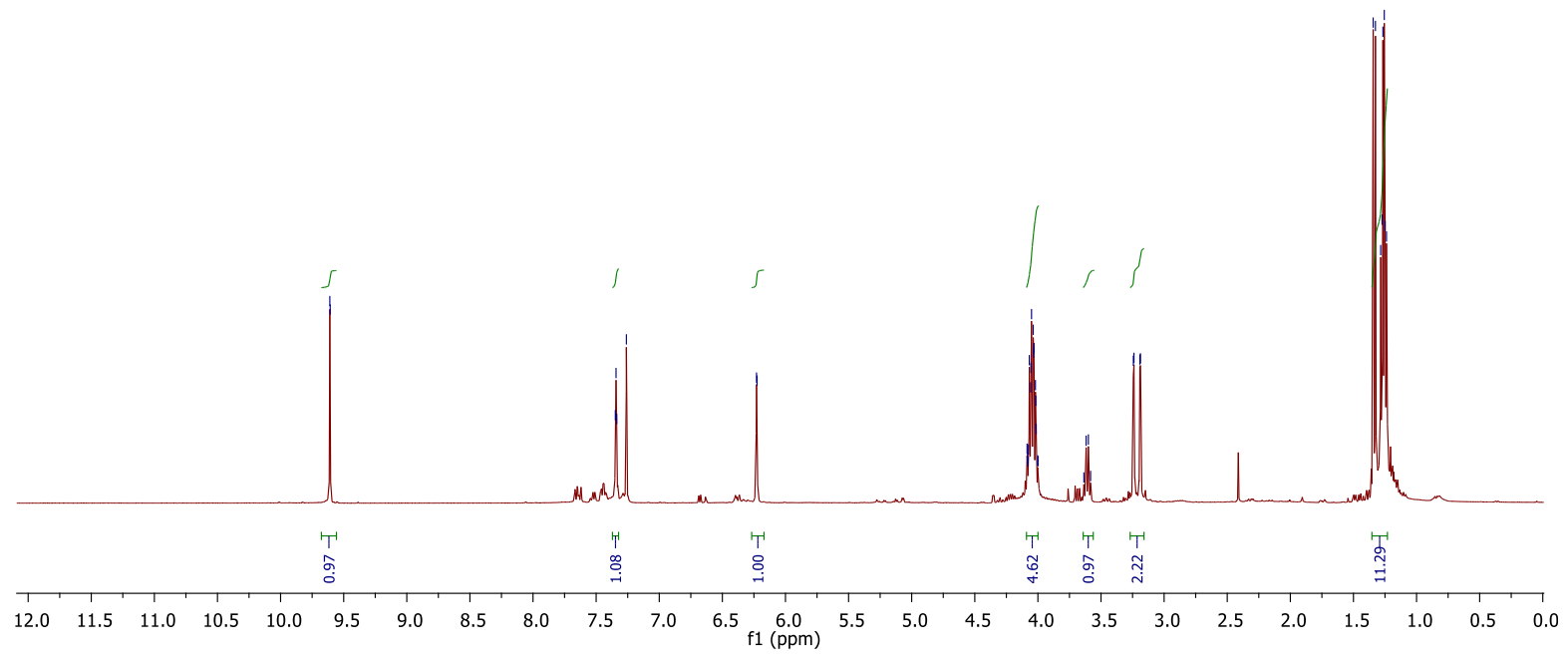

${ }^{13} \mathrm{C}-\mathrm{NMR}$ of compound $11 \mathrm{a}\left(101 \mathrm{MHz}^{\mathrm{CDCl}} \mathrm{CDC}_{3}\right)$
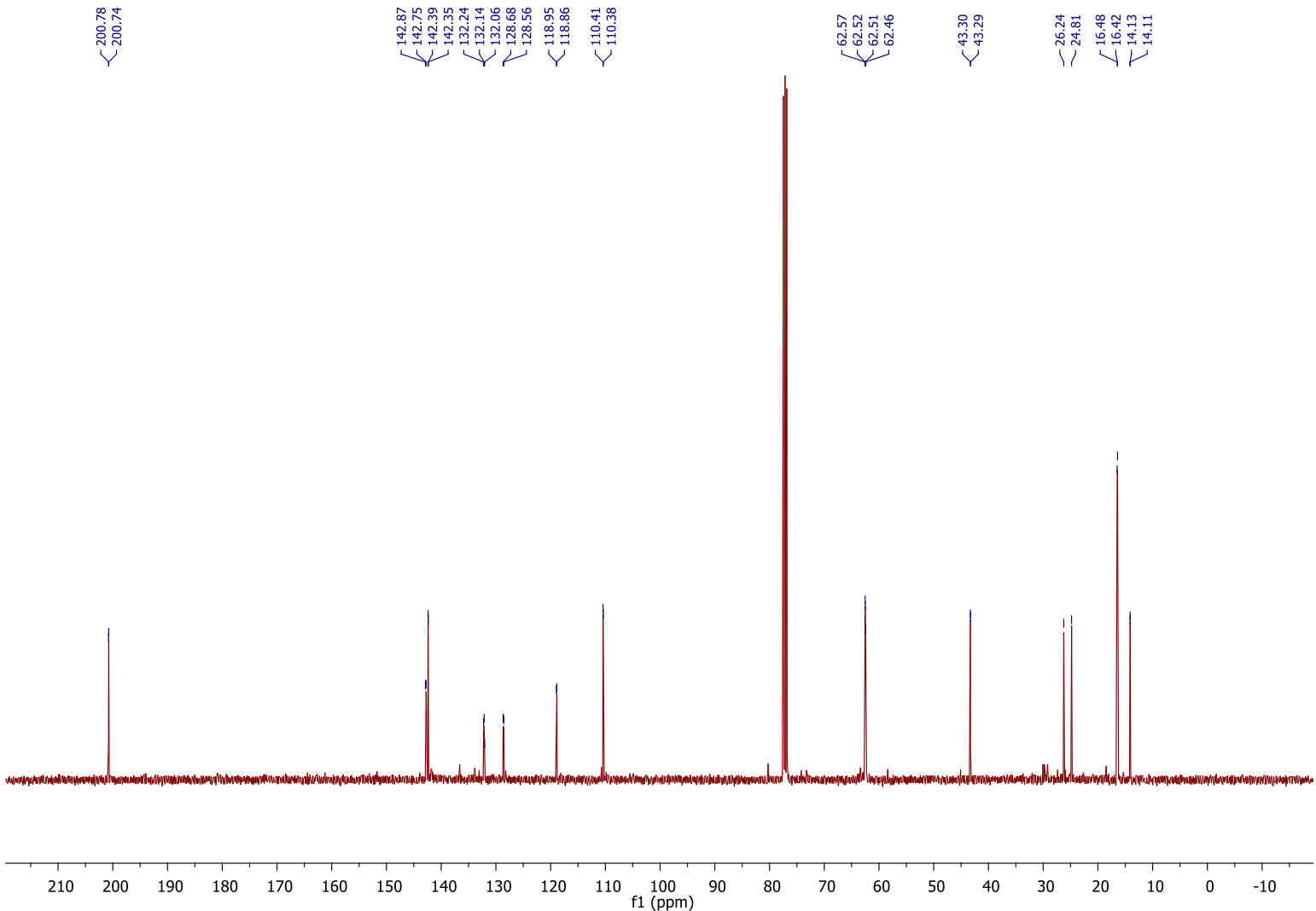


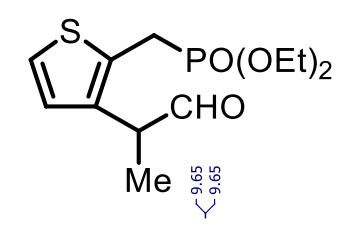

${ }^{1} \mathrm{H}-\mathrm{NMR}$ of compound $11 \mathrm{~b}\left(400 \mathrm{MHz}, \mathrm{CDCl}_{3}\right)$

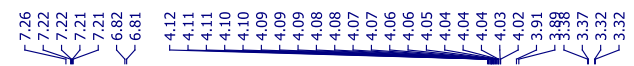

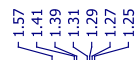

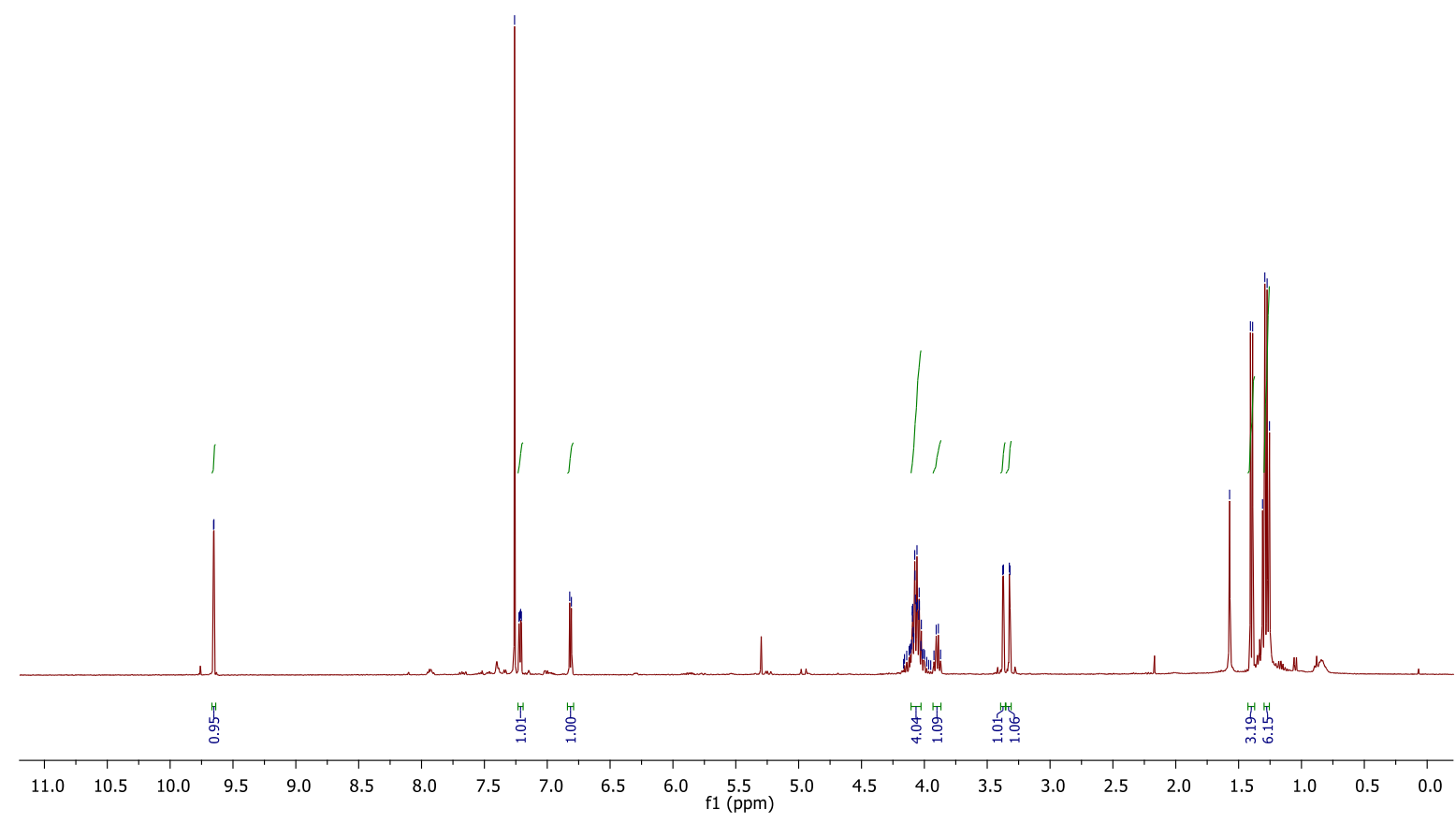

${ }^{13} \mathrm{C}-\mathrm{NMR}$ of compound $11 \mathrm{~b}\left(101 \mathrm{MHz}, \mathrm{CDCl}_{3}\right)$

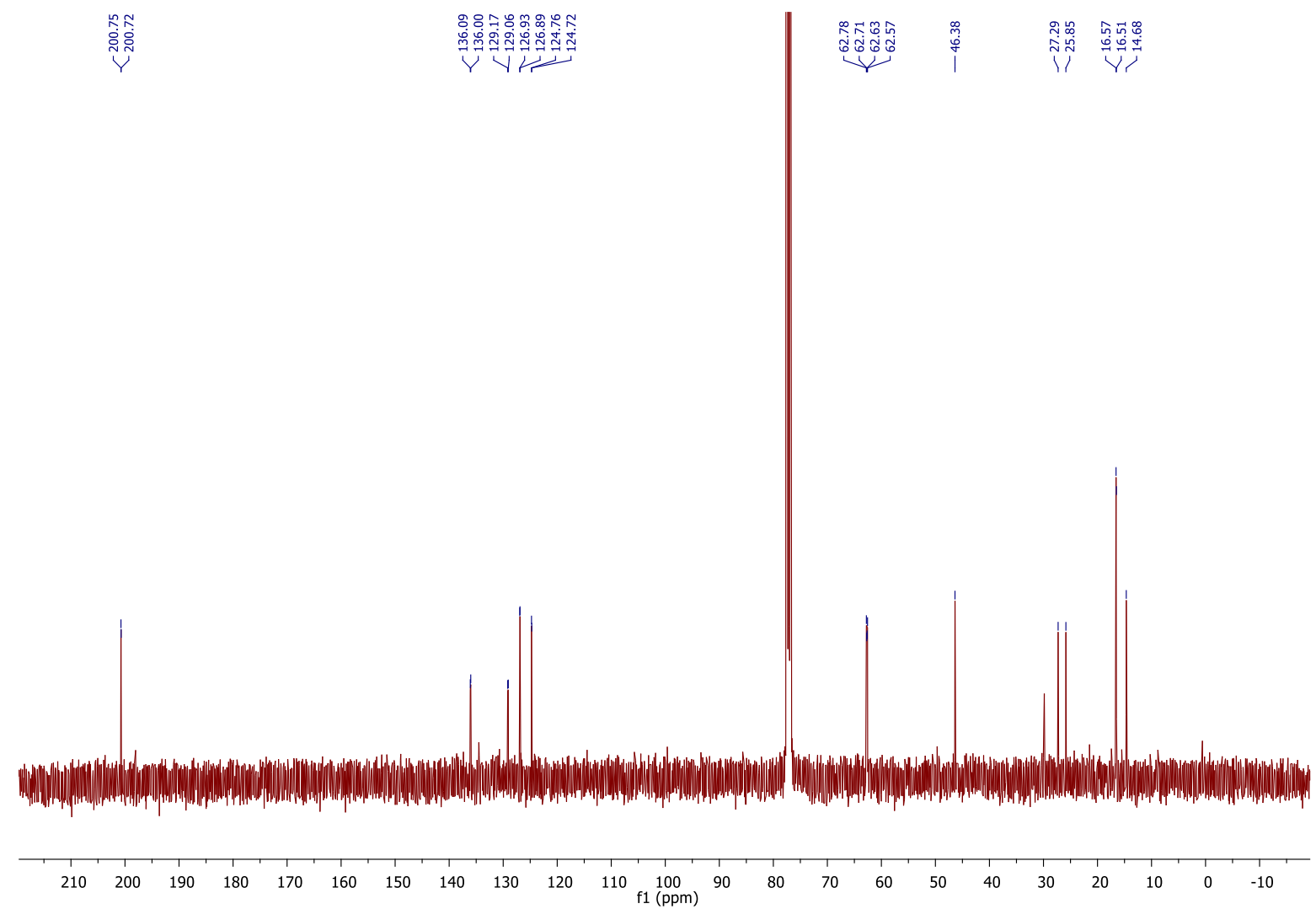


<smiles>CCOCc1ccoc1C(C)C=O</smiles>

${ }^{1} \mathrm{H}-\mathrm{NMR}$ of compound $\left.11 \mathrm{c}\left(400 \mathrm{MHz}^{\mathrm{CDCl}}\right)_{3}\right)$
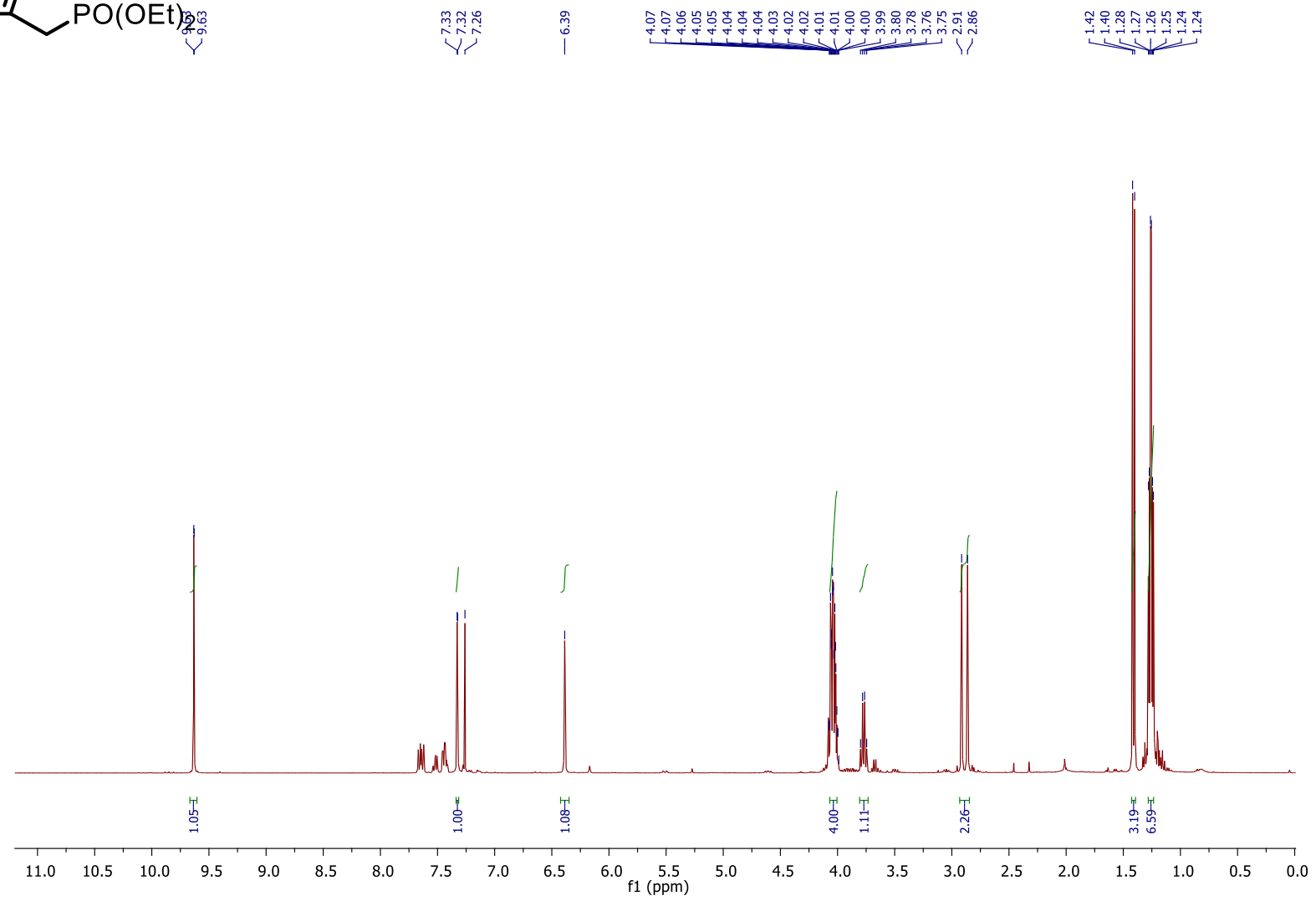

${ }^{13} \mathrm{C}-\mathrm{NMR}$ of compound $11 \mathrm{c}\left(101 \mathrm{MHz}, \mathrm{CDCl}_{3}\right)$

\begin{tabular}{|c|}
\hline 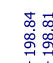 \\
\hline
\end{tabular}

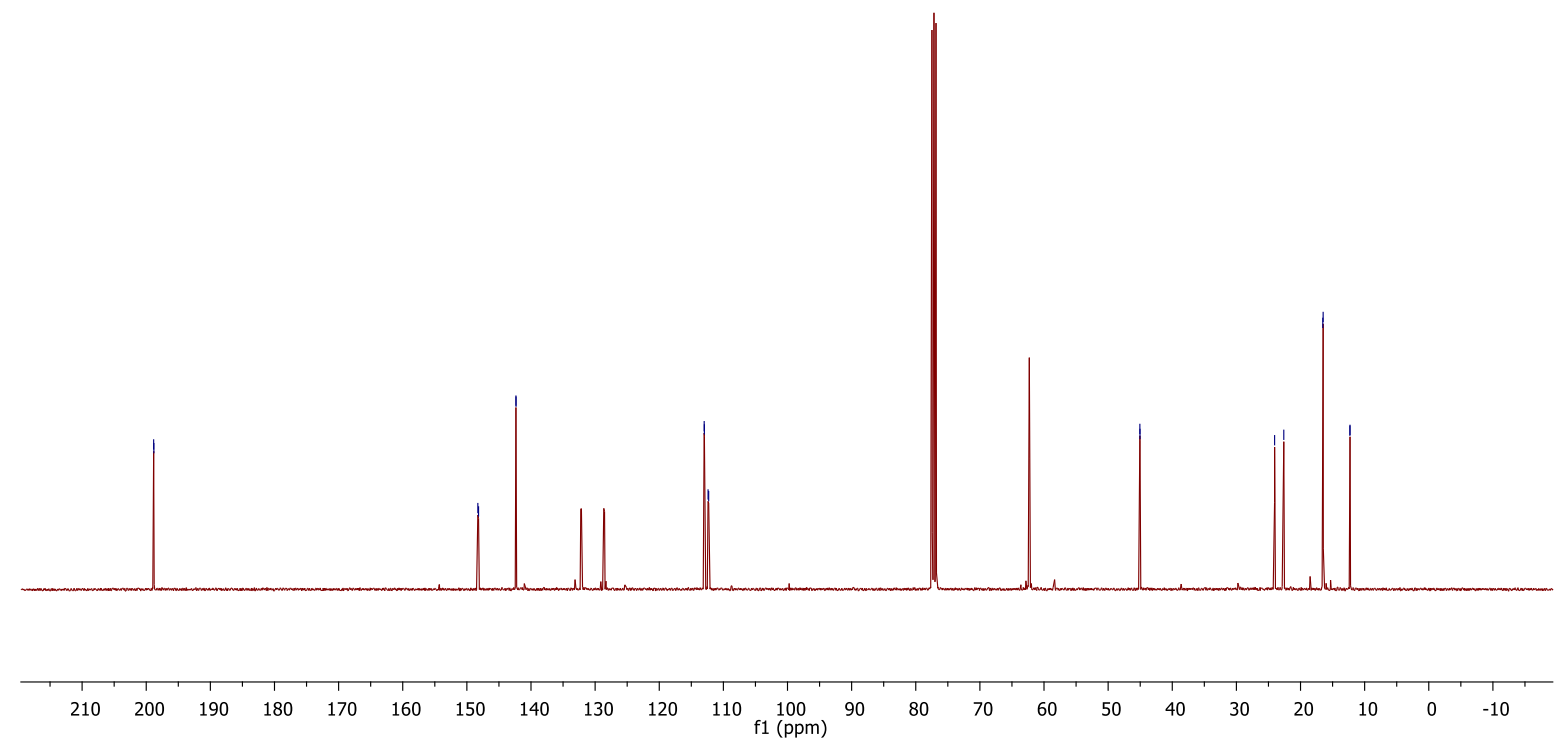




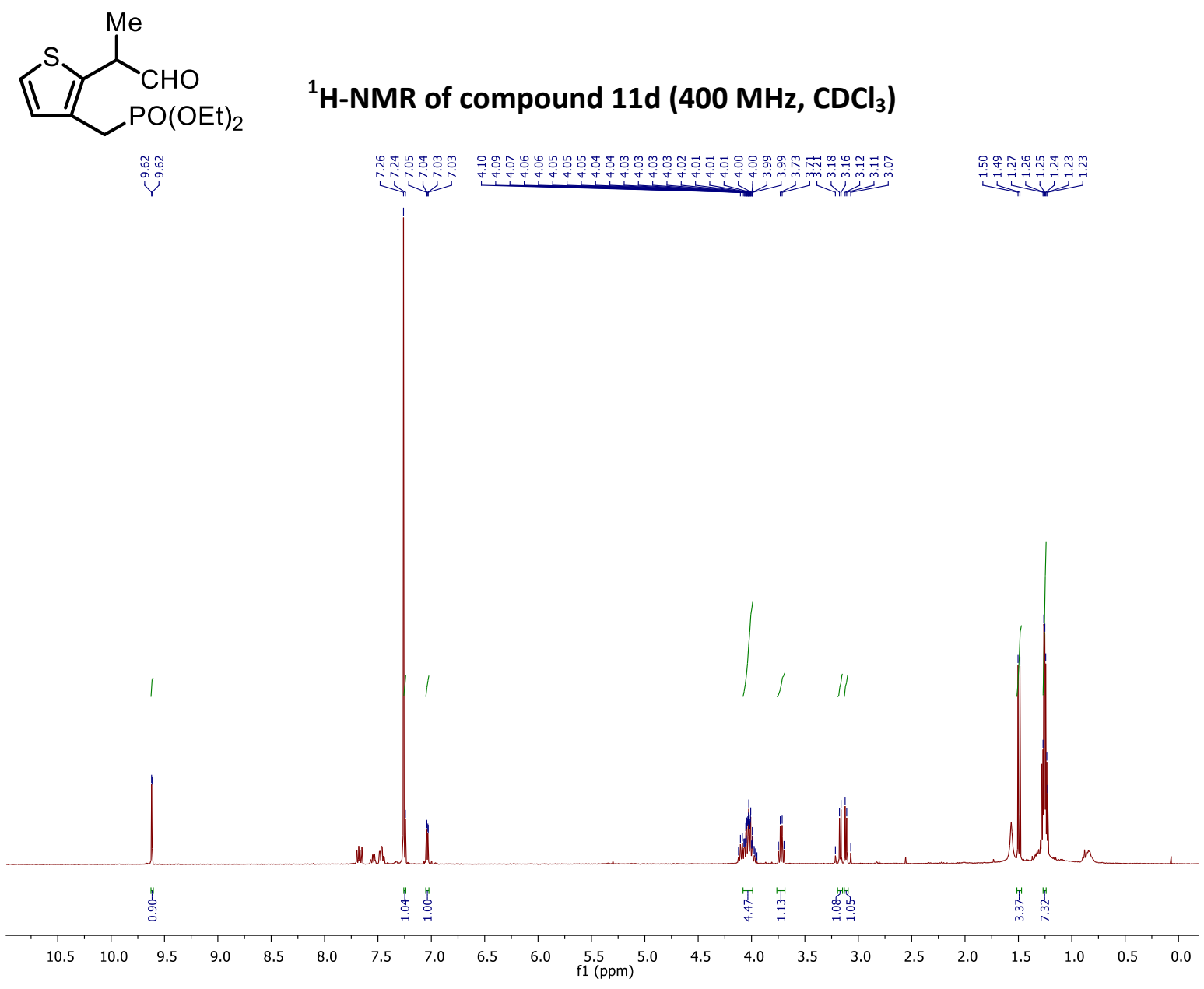

${ }^{13} \mathrm{C}$-NMR of compound $11 \mathrm{~d}$ (101 $\mathrm{MHz}, \mathrm{CDCl}_{3}$ )

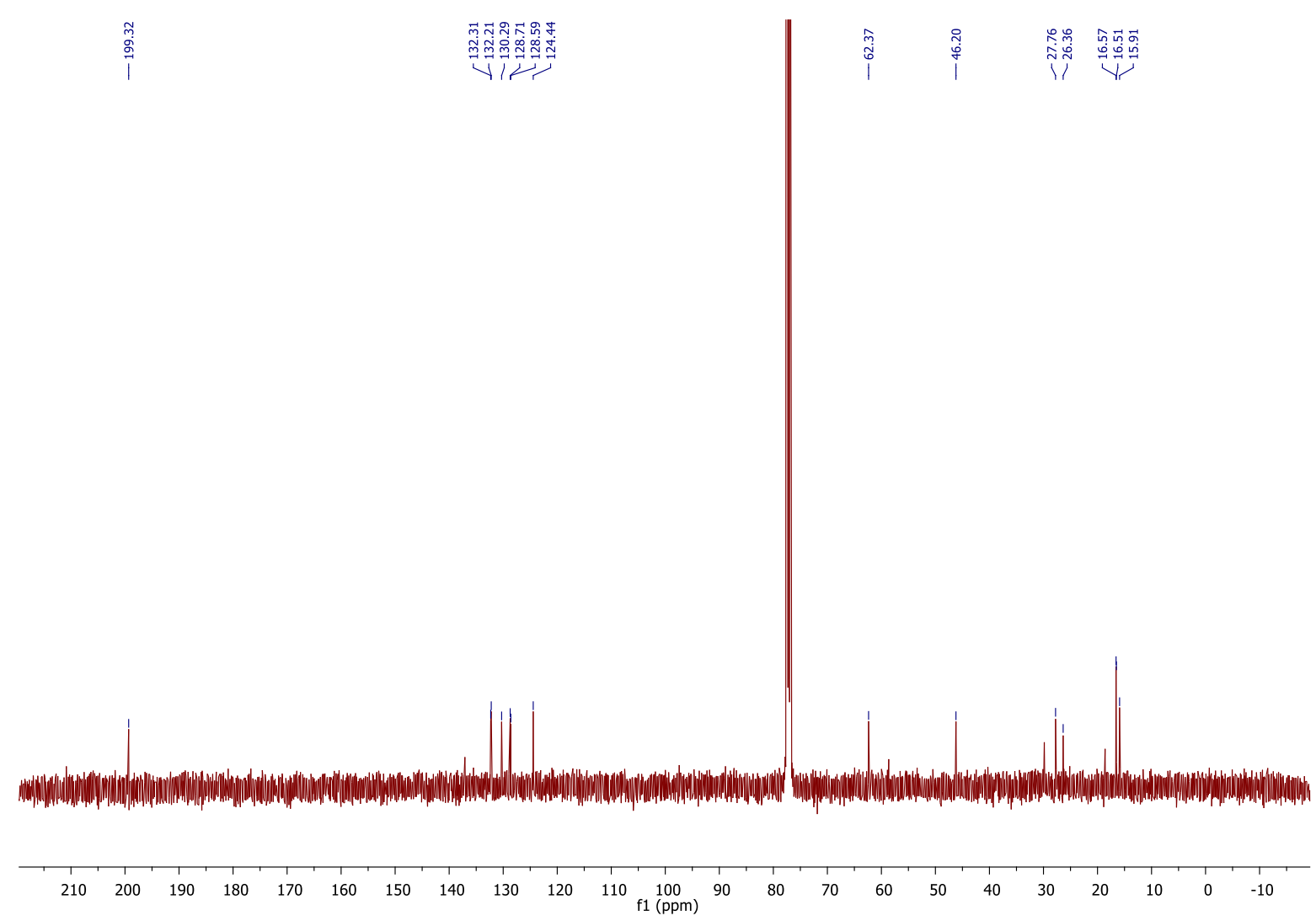


<smiles>CCOCc1sc2ccccc2c1C(C)CO</smiles>

${ }^{1} \mathrm{H}-\mathrm{NMR}$ of compound $11 \mathrm{e}\left(400 \mathrm{MHz}, \mathrm{CDCl}_{3}\right)$

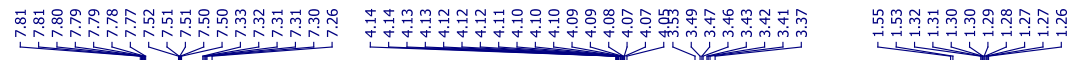

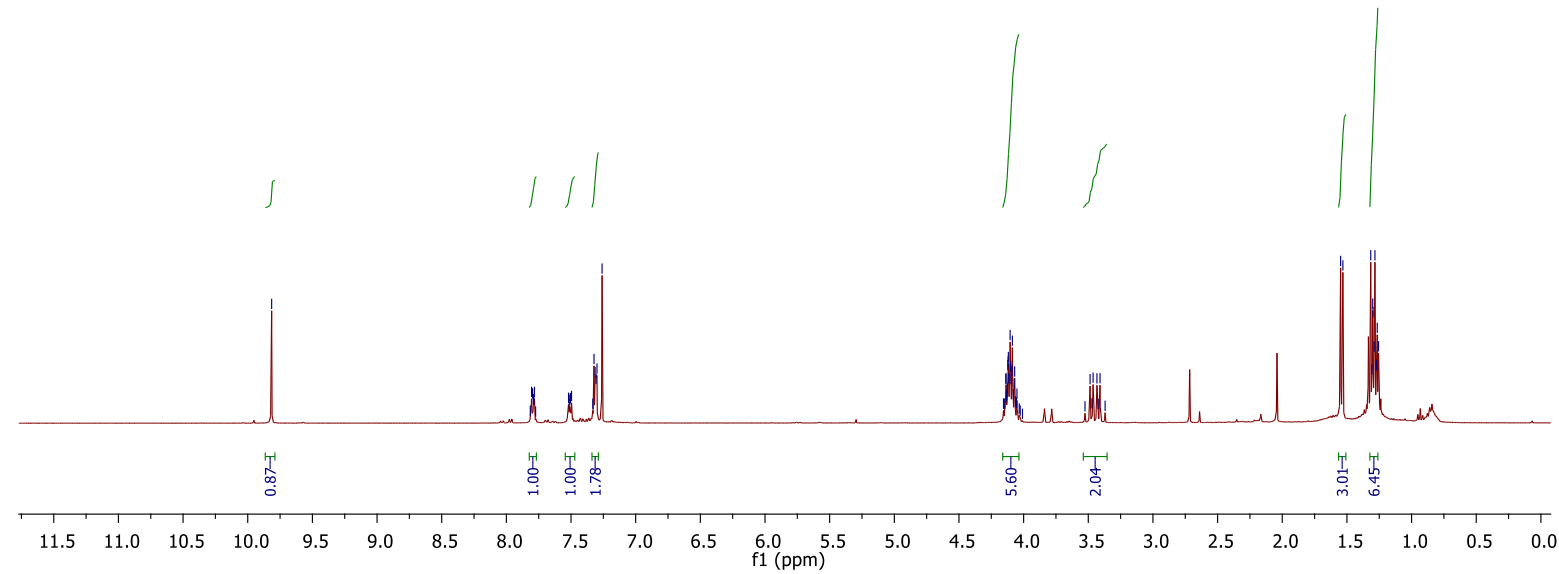

${ }^{13} \mathrm{C}-\mathrm{NMR}$ of compound $11 \mathrm{e}\left(101 \mathrm{MHz}, \mathrm{CDCl}_{3}\right)$

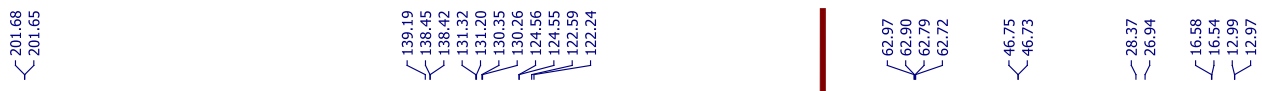

$\begin{array}{llllllllllllllllllllllllllll}210 & 200 & 190 & 180 & 170 & 160 & 150 & 140 & 130 & 120 & 110 & \begin{array}{c}100 \\ \mathrm{f} 1(\mathrm{ppm})\end{array} & 90 & 80 & 70 & 60 & 50 & 40 & 30 & 20 & 10 & 0 & -10\end{array}$ 


落

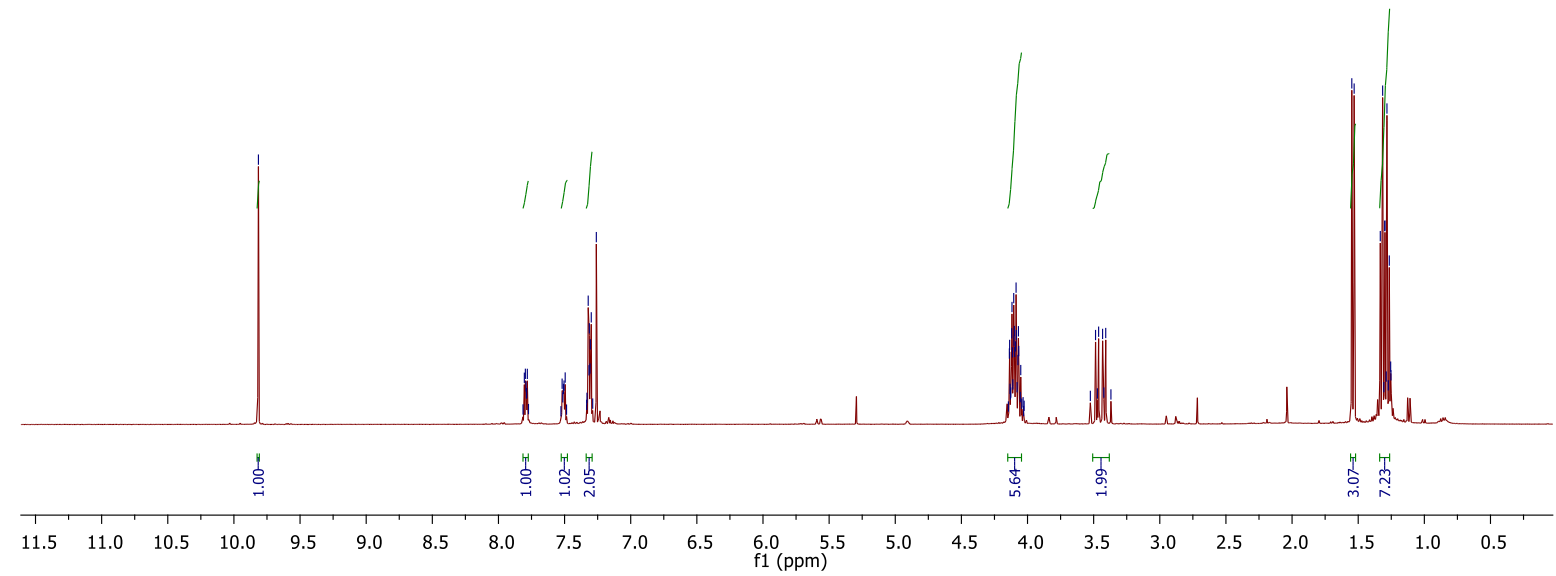

${ }^{13} \mathrm{C}-\mathrm{NMR}$ of compound $11 \mathrm{f}\left(101 \mathrm{MHz}, \mathrm{CDCl}_{3}\right)$

\begin{tabular}{|c|c|c|}
\hline & & 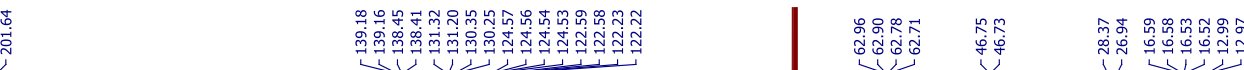 \\
\hline
\end{tabular}

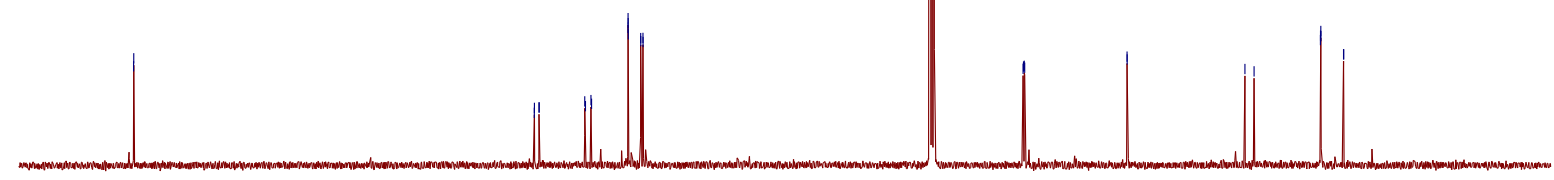

$\begin{array}{lllllllllllllllllllllll}210 & 200 & 190 & 180 & 170 & 160 & 150 & 140 & 130 & 120 & 110 & \underset{\mathrm{f} 1(\mathrm{ppm})}{100} & 90 & 80 & 70 & 60 & 50 & 40 & 30 & 20 & 10 & 0 & -10\end{array}$ 


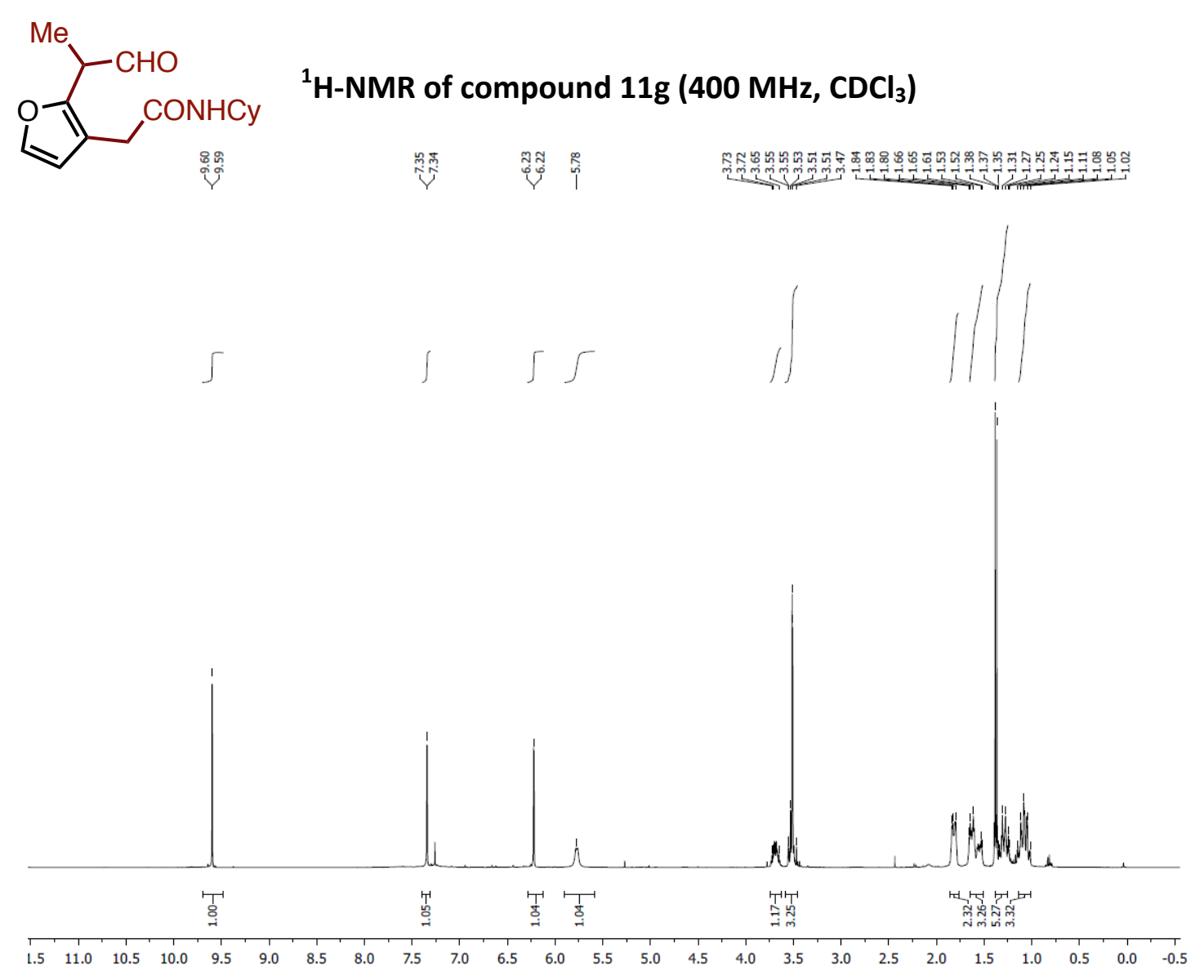

${ }^{13} \mathrm{C}-\mathrm{NMR}$ of compound $11 \mathrm{~g}\left(101 \mathrm{MHz}, \mathrm{CDCl}_{3}\right)$

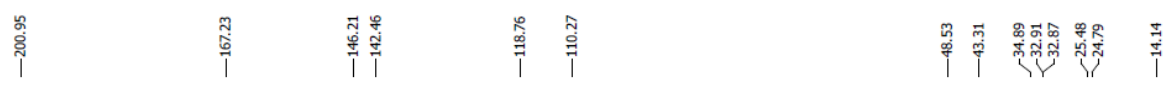

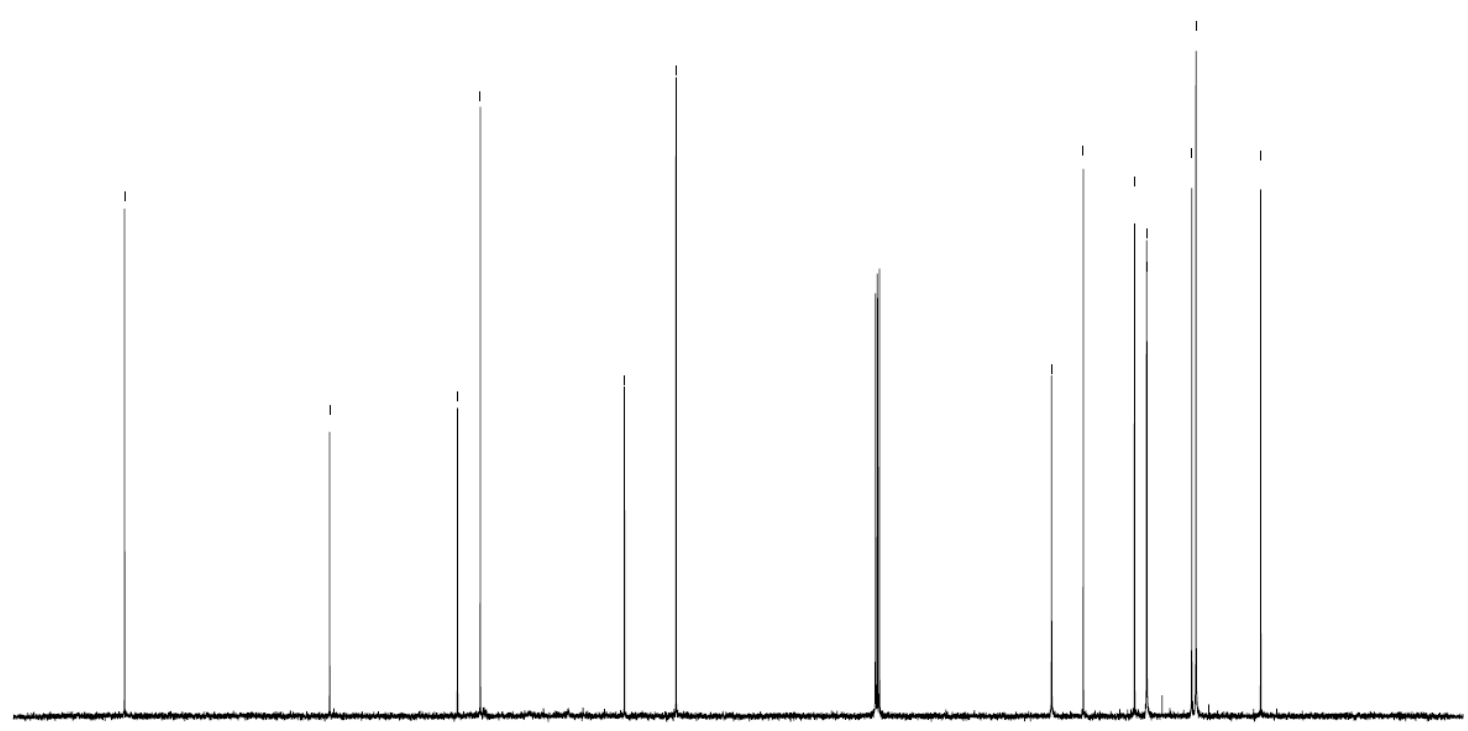

$\begin{array}{lllllllllllllllllllllll}210 & 200 & 190 & 180 & 170 & 160 & 150 & 140 & 130 & 120 & 110 & 100 & 90 & 80 & 70 & 60 & 50 & 40 & 30 & 20 & 10 & 0 & -10\end{array}$ 
<smiles>CCOCc1cnc2ccccc2c1C(C)CO</smiles>

${ }^{1} \mathrm{H}-\mathrm{NMR}$ of compound $11 \mathrm{~h}\left(400 \mathrm{MHz}, \mathrm{CDCl}_{3}\right)$

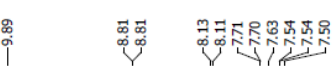

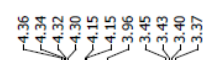

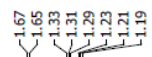
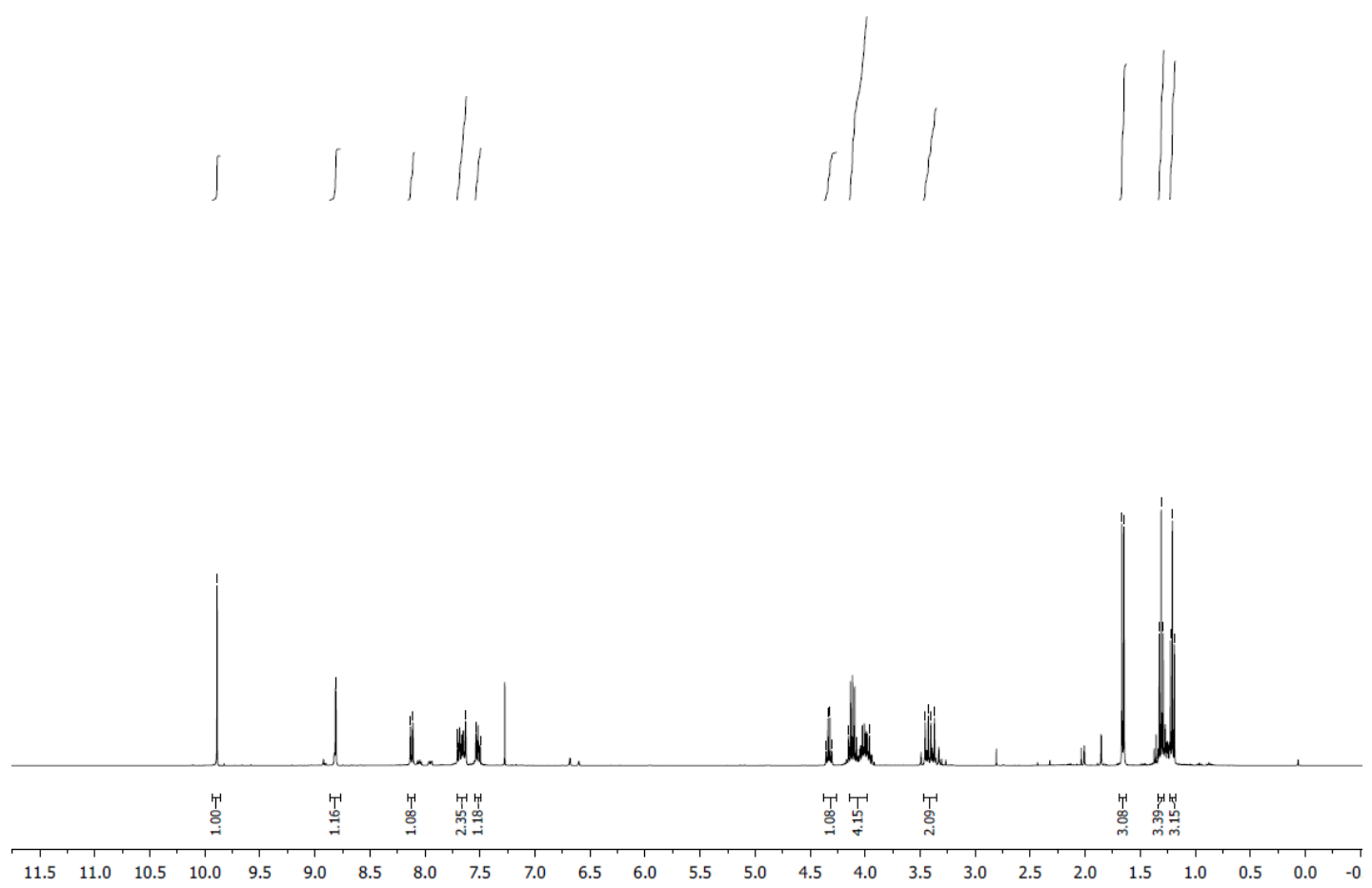

${ }^{13} \mathrm{C}-\mathrm{NMR}$ of compound $11 \mathrm{~h}\left(101 \mathrm{MHz}, \mathrm{CDCl}_{3}\right)$

ฟ้ำ

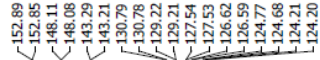

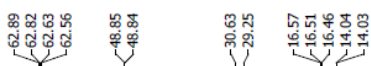

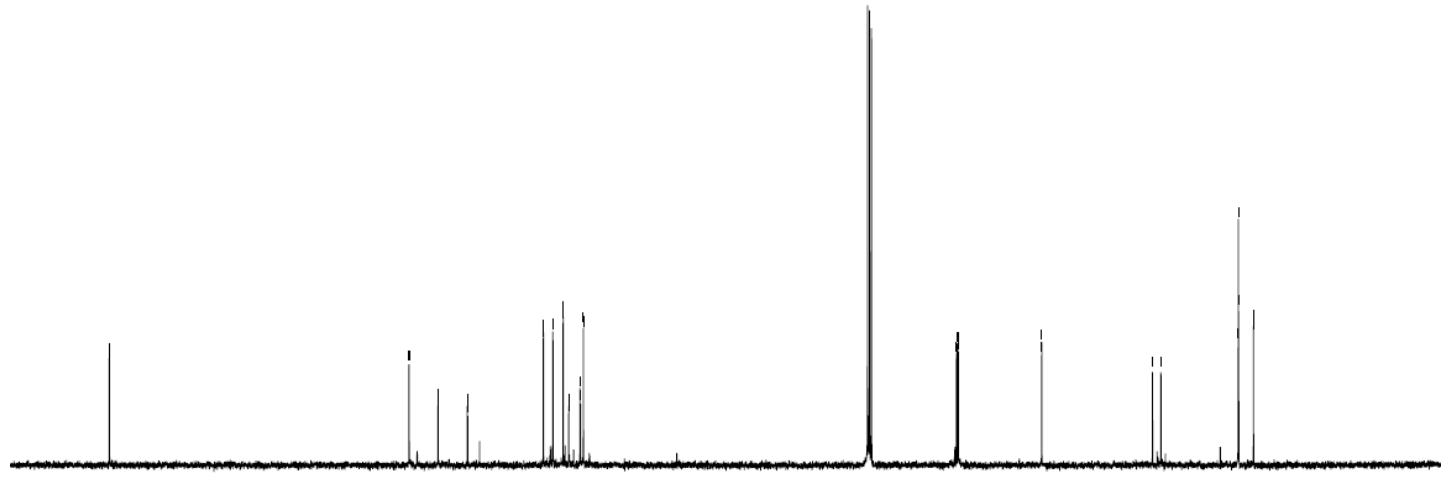

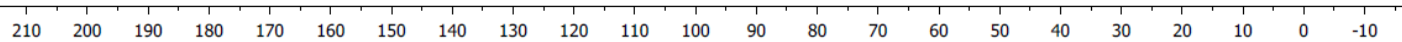

